\title{
Unboxing the brain
}

\author{
Citation for published version (APA):
}

Stevens, R. (2021). Unboxing the brain: development of technologies for non-invasive assessment of cerebral pathologies. [Doctoral Thesis, Maastricht University]. Gildeprint Drukkerijen. https://doi.org/10.26481/dis.20210115rs

Document status and date:

Published: 01/01/2021

DOI:

$10.26481 /$ dis.20210115rs

Document Version:

Publisher's PDF, also known as Version of record

\section{Please check the document version of this publication:}

- A submitted manuscript is the version of the article upon submission and before peer-review. There can be important differences between the submitted version and the official published version of record.

People interested in the research are advised to contact the author for the final version of the publication, or visit the DOI to the publisher's website.

- The final author version and the galley proof are versions of the publication after peer review.

- The final published version features the final layout of the paper including the volume, issue and page numbers.

Link to publication

\footnotetext{
General rights rights.

- You may freely distribute the URL identifying the publication in the public portal. please follow below link for the End User Agreement:

www.umlib.nl/taverne-license

Take down policy

If you believe that this document breaches copyright please contact us at:

repository@maastrichtuniversity.nl

providing details and we will investigate your claim.
}

Copyright and moral rights for the publications made accessible in the public portal are retained by the authors and/or other copyright owners and it is a condition of accessing publications that users recognise and abide by the legal requirements associated with these

- Users may download and print one copy of any publication from the public portal for the purpose of private study or research.

- You may not further distribute the material or use it for any profit-making activity or commercial gain

If the publication is distributed under the terms of Article $25 \mathrm{fa}$ of the Dutch Copyright Act, indicated by the "Taverne" license above, 


\section{UNBOXING THE BRAIN}

DEVELOPMENT OF TECHNOLOGIES FOR NON-INVASIVE Assessment of Cerebral Pathologies

Raoul Stevens 
Layout: Raoul Stevens

Design: Raoul Stevens

Printed by: Gildeprint

ISBN: $\quad$ 978-94-6419-086-1

(C)2021 Raoul Stevens

All rights reserved. No parts of this thesis may be reproduced, stored in a retrieval system or transmitted in any form or by any means without prior permission of the author.

Financial support for the publication of this thesis as provided by Maastricht University is gratefully acknowledged. 


\title{
UNBOXING THE BRAIN
}

\author{
DEVELOPMENT OF TECHNOLOGIES FOR NON-INVASIVE \\ ASS ESSMENT OF CEREBRAL PATHOLOGIES
}

\section{PROEFSCHRIFT}

Ter verkrijging van de graad van doctor aan de Universiteit Maastricht, op gezag van de Rector Magnificus, Prof. dr. Rianne M. Letschert, volgens het besluit van het College van Decanen, in het openbaar te verdedigen op vrijdag 15 januari 2021 om 12.00 uur

door

\section{Raoul Rudolf Florentius Stevens}

Geboren op 5 november 1990 Sittard, Nederland 


\section{Promotoren}

Prof. dr. T. Delhaas

Prof. dr. W.H. Mess

\section{Copromotor}

Dr. ir. W. Huberts

\section{Beoordelingscommissie}

Prof. dr. Y. Temel (voorzitter)

Dr. P. Bonizzi

Prof. dr. J.C.C. van der Horst

Prof. dr. ir. M. Mischi (Technische Universiteit Eindhoven)

Dr. S.C. Tromp (St. Antonius ziekenhuis Nieuwegein) 


Summary 
E A R LY diagnosis and monitoring of brain function is crucial to provide adequate healthcare to patients suffering from cerebral pathologies and injuries. Unfortunately, most existing monitoring methods are either invasive, not accurate enough, or limited by the restricted accessibility of the brain and, hence, need to be improved. Moreover, there are still many cerebral pathologies that cannot be monitored because the relevant metrics can simply not be measured. Technologies such as computer algorithms might be used to improve the accuracy of non-invasive assessments methods, whereas computational models that mimic physiological processes of the human body might be used to estimate clinical metrics by using and combining information that can be measured at other more accessible locations in the body.

In this thesis we have developed two new technologies to improve the noninvasive assessment of two different cerebral pathologies. In Part I of this thesis (Chapters 2 and 3) we address the non-invasive assessment of elevated intracranial pressure (ICP) by means of the optic nerve sheath diameter (ONSD) and develop an automated algorithm to obtain operator-independent metrics, whereas in Part II (Chapters 4 and 5) we focus on improving the estimation of patient-specific boundary conditions for cerebral aneurysm rupture risk models by using a $1 \mathrm{D}$ pulse wave propagation model (PWPM) to simulate pressure and flow waveforms that can often not be measured.

\section{Non-invasive ICP assessment using the ONSD}

Intracranial pressure assessment plays a crucial role in monitoring patients suffering from traumatic brain injury. Since existing ICP measurement techniques are very invasive, other ways to estimate ICP have been explored. The diameter of the optic nerve sheath (ONS) has proven to be a promising surrogate marker for ICP because the optic nerve sheath expands when ICP increases $[1,2]$ and its diameter can be measured from B-mode ultrasound images obtained by means of transorbital insonation. However, ONSD cut-off values for the detection of elevated ICP vary between studies [3-5], which hampers its clinical applicability.

In Chapter 2 we performed a review of the current literature on manual ONSD assessment methodologies to identify differences in ONSD assessment methodologies that could potentially cause the discrepancies in ONSD threshold values. Our review not only showed differences in the characteristic appearances of the B-mode ultrasound images, but also in the placement of the ultrasound markers used to denote the ONSD. Most importantly, the differences in ultrasound marker 
placement resulted in different ONSD values that also had varying sensitivities to changes in ICP. The chapter is concluded with a set of guidelines as a first step towards standardization of manual ONSD assessment to reduce the variations in ONSD values due to methodological differences

In Chapter 3 we took the standardization of ONSD assessment a step further by developing a fully automatic algorithm that is capable of segmenting the ONSD from B-mode ultrasound images. We demonstrated that the algorithm not only removed the intra- and interobserver variability associated with manual ONSD assessment, but also resulted in ONSD values that were comparable to the manual ONSD assessment performed by two experts. Using the presented algorithm, the variation within ONSD values decreases, which in turn, reduces the discrepancies within the ONSD threshold values and might lead to an improved stratification between patients with normal and elevated ICP.

\section{Estimation of cerebral boundary conditions}

The risk of aneurysm rupture has to be carefully balanced against the risk of complications associated with interventions. Computational fluid dynamics (CFD) models have proven to be capable of simulating rupture risk indices that can aid in clinical decision-making[6]. However, the accuracy of rupture risk indices is highly dependent on the boundary conditions (BCs) applied to the CFD simulations. Unfortunately, it is often not possible to measure patient-specific BCs within the clinical setting. As an alternative, BCs can be simulated using $1 \mathrm{D}$ pulse wave propagation models[7]. However, simulated $\mathrm{BC}$ s and corresponding rupture risk indices most likely depend on the input parameters and model assumptions of the PWPM. Therefore, the effects of the model input parameters and model assumptions on the $\mathrm{BC}$ and rupture risk indices have to be investigated.

In Chapter 4 we investigated the influence of inter-subject variations in our PWPM input parameters on simulated BCs and corresponding rupture risk indices that were derived from 3D cerebral aneurysm simulations. Our results showed that the inter-subject variations of the input parameters can lead to uncertainties within the rupture risk indices that are of the same order as the difference between ruptured and non-ruptured aneurysms and might therefore lead to misdiagnoses. These results highlight the importance of accurate and patient-specific BCs and the development of frameworks that can simulate these patient-specific BCs. 
In Chapter 5 we investigated the effect of the often discussed static pressurecoupling assumption at bifurcations [8] on our pressure and flow waveforms simulated with the PWPM. Although our results showed that changing the pressurecoupling hardly altered the $1 \mathrm{D}$ pressure and flow waveforms, it might be that pressure-coupling becomes important when the PWPM is adjusted to match patientspecific hemodynamic situations. Using the framework presented in Chapters 4 and 5 the effects of model assumptions and model input parameters on (possible) clinical metrics can be evaluated and used to optimize and individualize computer models that mimic physiological processes of the human body.

This thesis is concluded with a General Discussion (Chapter 6) where the results and main findings of the different chapters are put into a broader perspective. Moreover, the limitations of our research are discussed and we elaborate on how new technologies can contribute to the future of patient-specific clinical monitoring and decision-making. 


\section{Contents}

Summary vii

$\begin{array}{lll}\text { Chapter } 1 & \text { General Introduction } & 1\end{array}$

Part I Non-invasive ICP assessment using the ONSD

Chapter 2 Review \& Recommendations on ONSD Measurements 13

Chapter 3 Development of an Automatic ONSD Algorithm 35

Part II Estimation of cerebral boundary conditions

Chapter 4 Effect of Systemic Properties on Aneurysm Rupture Indices $\quad 63$

Chapter 5 A new OD Junction Element for Pressure Losses at Bifurcations 87

$\begin{array}{lll}\text { Chapter } 6 \text { General Discussion } & \mathbf{1 1 5}\end{array}$

Appendices

Valorization

133

Acknowledgements (Dankwoord)

139

About the author 



\section{CHAPTER}

\section{General Introduction}




\subsection{Focus of this thesis}

I $\mathrm{N}$ this thesis we aim to develop and evaluate technologies to improve noninvasive assessment of cerebral pathologies. Specifically, we evaluate: 1) the use _of automatic segmentation algorithms to assess the optic nerve sheath diameter as surrogate marker for elevated intracranial pressure, and 2) the use of a pulse wave propagation model for the definition of proper boundary conditions for threedimensional rupture risk prediction models of cerebral aneurysms.

This chapter starts with describing the anatomy of the brain, and the production and function of cerebrospinal fluid (CSF), followed by a description of the cerebral blood supply and its regulation required to maintain cerebral functions. Thereafter, the clinical backgrounds and existing diagnostic and prognostic methods are introduced for both patients with traumatic brain injury and patients with cerebral aneurysms. Finally, the rationale and the specific objectives addressed in this thesis are given.

\subsection{Clinical background}

\subsubsection{Brain anatomy and cerebrospinal fluid}

The human brain is encapsulated by three meningeal layers and the skull. The three meningeal layers from inside to outside are the pia mater, the arachnoid mater, and the dura mater $[1,2]$. The subarachnoid space, located between the pia and the arachnoid mater, contains CSF. The CSF acts as a shock absorber, allows for distribution and regulation of nutrients and waste products between the neurons, and plays an important role in maintaining cerebral blood flow $[1,3]$. CSF is produced at the choroid plexus inside the brain ventricles. From here, it passes into the subarachnoid space around the brain and spinal cord via the median and lateral apertures[3]. Finally, it is absorbed into the rigid sinuses via arachnoid granulations $[1,2]$. These sinuses then drain the CSF into veins that return the blood to the heart. The total volume of the brain $(\sim 1700 \mathrm{ml})$ is enclosed by the skull and consists of the following components: brain tissue $(\sim 1400 \mathrm{ml})$, blood $(\sim 150 \mathrm{ml})$, and CSF $(\sim 150 \mathrm{ml})$ [3]. The total intracranial pressure (ICP) inside the skull normally lies between 10 and $15 \mathrm{mmHg}[3,4]$.

A continuously regulated ICP is crucial for adequate brain perfusion as the perfusion is driven by the cerebral perfusion pressure (CPP), i.e. the pressure difference between the arterial blood pressure and the ICP [4]. In the healthy situation a compensatory mechanism, known as the Monro-Kellie doctrine, ensures an approximately constant ICP by compensating a volume increase in one of the three aforementioned components with an equal volume decrease in one or more of the other components[4]. For example, an abnormal bleeding increases the blood 
volume within the skull which will be compensated by an increased CSF drainage via the dural sinuses or shifting of CSF into the spinal subarachnoid space[2].

\subsubsection{Cerebral vasculature and blood flow regulation}

The human brain is responsible for almost $20 \%$ of the body's total oxygen and glucose consumption, although its weight is only $2 \%$ of the total body mass[1]. Disruption of blood flow and oxygen supply to the brain leads to unconsciousness within seconds and to irreversible brain damage within minutes because glucose and oxygen cannot be stored in the brain. To allow a continuous supply of blood the cerebral circulation and the regulation of cerebral blood flow are crucial. The brain has two important mechanisms to maintain adequate blood and nutrient supply to all areas of the brain at all time: i.e. 1) collateral blood flow pathways, and 2) blood flow regulation.

The brain is provided with $750 \mathrm{ml}$ of blood per minute (approximately $15 \%$ of the cardiac output) [5, 6] via two sets of arteries, i.e. the left and right internal carotid arteries (ICA) and the left and right vertebral arteries. The latter ones merge into the basilar artery (BA). At the base of the brain the ICAs and BA merge into a ring-like structure called the Circle of Willis, which is the largest example of a collateral pathway in the brain. It divides the blood over the anterior, middle, and posterior parts of the brain via the anterior, middle, and posterior cerebral arteries. After the blood passes the capillary beds, where oxygen, carbon dioxide, nutrients, and waste products are exchanged, it is drained into rigid sinuses via collapsible cerebral veins. Together with the absorbed CSF it is then returned to the heart via the internal jugular veins.

The amount and distribution of blood across different regions of the brain is actively regulated by changing vascular resistances. This allows for an increase in blood flow to specific areas of the brain when the demand is increased (neuro-vascular coupling and metabolic regulation). Furthermore, it ensures that the cerebral blood flow remains constant when blood pressure increases or drops (myogenic regulation)[7]. Changes in vascular resistances can be achieved by rapid vasoconstriction or vasodilation due to contraction or relaxation of smooth muscles cells (SMC) primarily located in the arteriolar walls. When the demand of blood rises, arteriolar vasodilation will lead to a reduction of vascular resistance which allows the blood flow to rise. An increase of the (perfusion) pressure, on the other hand, will induce vasoconstriction to keep the flow at a constant level. Finally, a sudden decrease in blood pressure, which would result in a reduced blood flow to the brain, can be compensated by a quick enlargement of the arteriolar radii. 


\subsection{Traumatic brain injury}

\subsubsection{Hemodynamic changes in traumatic brain injury}

Brain swelling and edema can occur in patients suffering from traumatic brain injury (TBI) $[8,9]$ due to for example, a blow to the head. When this increase in volume cannot be compensated for by reducing the volume of blood or CSF, ICP increases[9]. Assuming the systemic blood pressure remains the same, the CPP will decrease which reduces the perfusion of the brain[8]. If ICP increases even further, the venous side of the circulation will collapse significantly which impairs the outflow of blood from the intracranial space. This will drive the ICP even higher and at some point the perfusion of the cerebral tissue will stop completely. To ensure sufficient perfusion and prevent irreversible tissue damage in TBI patients, it is crucial to normalize their ICP[10].

\subsubsection{Non-invasive assessment of intracranial pressure}

Intracranial pressure assessment is important because it represents the severity of the brain injury[4, 9]. Moreover, continuous or frequent monitoring of the ICP allows clinicians to evaluate the effectiveness of their treatment[9]. Unfortunately, performing ICP measurements within the brain is challenging as the cranium makes it almost inaccessible. Consequently, the ICP can only be measured by drilling a hole in the skull and placing a drain or a pressure sensor in respectively, the ventricles or the brain parenchym $[4,6]$. It goes without saying that such a procedure is highly invasive and comes with a large risk of complications. To prevent this risk of complications and to obtain more information about the hemodynamic status inside the brain, there is an unmet need for new and preferably non-invasive assessment methods.

Although ICP cannot be measured directly without entering the skull, the optic nerve sheath diameter (ONSD) can be used as a non-invasive surrogate marker of ICP[11-14]. The sheath surrounding the optic nerve contains CSF that is connected to the CSF surrounding the brain. As a consequence, the diameter of the optic nerve sheath enlarges when the ICP increases. The ONSD can be estimated on transocular ultrasound B-mode images. Current clinical practice is to manually assess ONSD from these images using ultrasound callipers. By linking these ONSD measurements to invasively measured ICP values, regression models can be created that can be used to estimate the ICP non-invasively. Unfortunately, ONSD cut-off values for the diagnosis of increased ICP are inconsistent between studies[15-17], which hampers the implementation of ONSD as a possible non-invasive bedside monitoring tool for ICP. Likely sources of these discrepancies are: 1) differences in ONSD assessment methodologies between centres, and 2) the manual ONSD 
assessment which might result in considerably inter- and intraobserver variability. Standardization of ONSD assessment methodologies and the development of an automatic ONSD assessment algorithm could overcome both problems and possibly reduce the inconsistency in ONSD cut-off values.

\subsection{Cerebral aneurysms}

\subsubsection{Hemodynamic changes in cerebral aneurysm}

In patients suffering from cerebral aneurysms weak spots in the arterial walls bulge due to forces delivered by the blood flow and associated pressure pulses. As a consequence of this dilation, the aneurysm wall becomes thinner which can cause the wall to become even weaker. When stresses in the vessel wall exceed the strength of the wall, rupture will occur. Though the initial widening of the blood vessel can remain asymptomatic and, hence, go unrecognized by the patient, a ruptured aneurysm puts the patient in an acute and a potentially life-threatening situation. Not only will blood leak out of the vessel thereby jeopardizing the blood supply to the brain, but the presence of blood in the subarachnoid space can also cause inflammation, edema, delayed cerebral ischemia, and stroke. Because preventive treatment of an aneurysm by coiling or stenting is also not without danger, the risk of the intervention has to be assessed and weighed against the risk of rupture[18].

\subsubsection{Non-invasive assessment of aneurysm rupture risk}

To assess whether a patient with an aneurysm has to be treated, the aneurysm rupture risk has to be determined. Unfortunately, this rupture risk cannot be measured directly. Although non-invasive morphological metrics such as the diameter[19-22], have been correlated with aneurysm rupture risk, none of these can accurately stratify aneurysm rupture risk. Therefore, researchers have started focussing on hemodynamic factors, such as wall shear stress (WSS), that affect aneurysm growth and rupture[19, 20, 23-25]. Computational Fluid Dynamics (CFD) models have the ability to simulate the hemodynamics in and around the aneurysm and are therefore often used to calculate hemodynamic metrics related to aneurysm rupture in order to approximate the aneurysm rupture risk. These CFD simulations require knowledge of the following two characteristics: 1 ) the geometry of the aneurysm, and 2) proper boundary conditions (BCs). Though the $3 \mathrm{D}$ geometry of the aneurysm and the surrounding vessels can be obtained using 3DRA or CTA[26], the BCs for the 3D domain can often not be measured in vivo. In this absence of patient-specific boundary conditions the best alternative is the simulation of BCs using 1D pulse wave propagation models (PWPM)[27]. 
However, pressure and flow waveforms not only vary between patients, but also within patients due to for example, daily activities. Boundary conditions for 3D models must resemble these patients' waveforms closely in order to obtain accurate predictions of flow waveforms in and around the aneurysm[28]. The inter- and intrasubject variabilities must therefore be incorporated in the PWPM by setting model parameters to patient-specific values. Unfortunately, measuring these values in patients is not always possible, and if possible, it is time consuming. However, it might not be necessary to make the whole model patient-specific because not all model parameters might be equally important for the estimation of correct BCs. Uncertainty and sensitivity quantification needs to be performed to find out which model parameters should be set to patient-specific values and therefore require patient-specific measurements.

\subsection{Aims and Thesis outline}

Current non-invasive approaches for the monitoring of the two cerebral pathologies described above are promising but cannot yet compete with current gold standards for clinical monitoring and decision-making.

The aim of this thesis is to develop and evaluate new technologies to improve the existing non-invasive monitoring and decision-making approaches.

This thesis is structured in two parts. Part I (Chapters 2 and 3) deals with improving the assessment of optic nerve sheath diameter as non-invasive surrogate for intracranial pressure. Part II (Chapters 4 and 5) evaluates boundary conditions for CFD simulations of cerebral aneurysms that are estimated by a 1D pulse wave propagation model.

\subsubsection{Part I: Non-invasive ICP assessment using the ONSD}

In Chapter 2 a review of the literature on ONSD assessment is performed to obtain insight in the different ONSD assessment methodologies used in clinical centres and research facilities. We investigate if discrepancies in assessment methodologies lead to different ONSD values, and if the methods vary in sensitivity to changes in ICP. In Chapter 3 the obtained insights are used to develop an automatic ONSD algorithm. The performance of the algorithm is quantified by comparing algorithm-derived ONSD values with manually-derived ONSD values. 


\subsubsection{Part II: Estimation of cerebral boundary conditions}

In Chapter 4 we assess the influence of inter-subject variations on wall shear stress indices of cerebral aneurysms. Hereto, we create a set of cerebral BCs by introducing population-based variations in systemic properties of our 1D PWPM. Next, we apply these BCs to 3D-CFD simulations of an aneurysm from which we calculate the corresponding aneurysm rupture indices. We then evaluate the effect of the variations in the input parameters on the BCs using uncertainty quantification. Moreover, we attribute the contribution of each individual parameter and its interactions with other parameters to the total uncertainty using sensitivity analysis. This allows us to identify parameters that should be set to patient-specific values in order to estimate proper boundary conditions for rupture risk assessment models.

The accuracy of our BCs also depends on the validity of the model assumptions of our PWPM, and there is debate on the correct pressure continuity assumption at bifurcations. Some researchers choose to ignore the extra pressure losses caused by the creation of vortices at bifurcations and assume continuity of the total pressure $\left(p+\frac{1}{2} \rho u^{2}\right)[29-31]$. Others, including ourselves, model only continuity of static pressure because they assume that both the change in dynamic pressure $\left(\frac{1}{2} \rho u^{2}\right)$, caused by the deceleration of blood, and the additional pressure loss can be neglected. They support this assumption by addressing the fact that these two terms are much smaller than the static pressure and that they have an opposite effect on the pressure drop $[32,33]$. However, it remains unclear whether these assumptions still hold in arterial networks with a large number of consecutive bifurcations such as in the cerebral vasculature[34]. In Chapter 5, we therefore develop a new bifurcation element that allows extending our 1D model with these two missing pressure terms. Using this model, we assess the influence on pressure and flow waveforms when using continuity of static pressure instead of total pressure.

Finally, the thesis is closed in Chapter $\mathbf{6}$ with a general discussion where the main findings of the different chapters will be discussed and put in a broader perspective. 


\section{References}

1. Tortora, G. \& Derrickson, B. Principles of anatomy and physiology 9, 1689-1699 (Johm Wiley \& Sons, Inc, 2016).

2. Smith, G. E. The Human Brain. Journal of Mental Science 76, 245-249 (1930).

3. Sakka, L., Coll, G. \& Chazal, J. Anatomy and physiology of cerebrospinal fluid. European Annals of Otorhinolaryngology, Head and Neck Diseases 128, 309-316 (2011).

4. Rangel-Castillo, L., Gopinath, S. \& Robertson, C. S. Management of Intracranial Hypertension. Neurologic Clinics 26, 521-541 (2008).

5. Xing, C.-y., Tarumi, T., Liu, J. et al. Distribution of cardiac output to the brain across the adult lifespan. Journal of Cerebral Blood Flow \& Metabolism 37, 2848-2856 (2017).

6. Rodríguez-Boto, G., Rivero-Garvía, M., Gutiérrez-González, R. \& Márquez-Rivas, J. Basic concepts about brain pathophysiology and intracranial pressure monitoring. Neurología (English Edition) 30, 16-22 (2015).

7. Paulson, O. B., Hasselbalch, S. G., Rostrup, E. \& Knudsen, G. M. Cerebral blood flow response to functional activation. Journal of Cerebral Blood Flow \& Metabolism Metabolism 30, 2-14 (2009).

8. Vella, M. A., Crandall, M., Patel, M. B. et al. Acute management of TBI. Surg Clin North Am 97, 1015-1030 (2017).

9. Galgano, M., Toshkezi, G., Qiu, X., Russell, T., Chin, L. \& Zhao, L. R. Traumatic brain injury: Current treatment strategies and future endeavors. Cell Transplantation 26, 1118-1130 (2017).

10. Stein, D. M., Feather, C. B. \& Napolitano, L. M. Traumatic Brain Injury Advances. Critical Care Clinics 33, 1-13 (2017).

11. Hansen, H. C. \& Helmke, K. Validation of the optic nerve sheath response to changing cerebrospinal fluid pressure: Ultrasound findings during intrathecal infusion tests. Journal of Neurosurgery 87 , 34-40 (1997).

12. Geeraerts, T., Merceron, S., Benhamou, D. et al. Non-invasive assessment of intracranial pressure using ocular sonography in neurocritical care patients. eng. Intensive Care Medicine 34, 2062-2067 (2008).

13. Toscano, M., Spadetta, G., Pulitano, P. et al. Optic Nerve Sheath Diameter Ultrasound Evaluation in Intensive Care Unit: Possible Role and Clinical Aspects in Neurological Critical Patients' Daily Monitoring. BioMed Research International 2017, 1-7 (2017).

14. Soldatos, T., Karakitsos, D., Chatzimichail, K., Papathanasiou, M., Gouliamos, A. \& Karabinis, A. Optic nerve sonography in the diagnostic evaluation of adult brain injury. Critical Care 12, 1-7 (2008).

15. Dubourg, J., Javouhey, E., Geeraerts, T., Messerer, M. \& Kassai, B. Ultrasonography of optic nerve sheath diameter for detection of raised intracranial pressure: A systematic review and metaanalysis. eng. Intensive Care Medicine 37, 1059-1068 (2011).

16. Wang, L.-J. J., Yao, Y., Feng, L.-S. S. et al. Noninvasive and quantitative intracranial pressure estimation using ultrasonographic measurement of optic nerve sheath diameter. eng. Scientific Reports 7, 1-7 (2017).

17. Pichamuthu, K. K. \& Prithishkumar, I. J. Appearance of the Optic Nerve Sheath Diameter (ONSD) using Higher Frequency Linear Probes in Detection and Monitoring of Raised Intracranial Pressures-A Cadaveric Study. Journal of Clinical and Diagnostic Research, 10-13 (2019). 
18. Cebral, J. R., Castro, M. a., Appanaboyina, S., Putman, C. M., Millan, D. \& Frangi, A. F. Efficient pipeline for image-based patient-specific analysis of cerebral aneurysm hemodynamics: Technique and sensitivity. IEEE Transactions on Medical Imaging 24, 457-467 (2005).

19. Detmer, F. J., Chung, B. J., Jimenez, C., Hamzei-sichani, F. \& Kallmes, D. Associations of hemodynamics , morphology , and patient characteristics with aneurysm rupture stratified by aneurysm location. Neuroradiology Journal 61, 275-284 (2019).

2o. Jing, L., Fan, J., Wang, Y., Li, H., Wang, S. \& Yang, X. Morphologic and Hemodynamic Analysis in the Patients with Multiple Intracranial Aneurysms : Ruptured versus Unruptured. PLoS ONE, 1-12 (2015).

21. Xiang, J., Natarajan, S. K., Tremmel, M. et al. Hemodynamic-Morphologic Discriminants for Intracranial Aneurysm Rupture. Stroke 42, 144-152 (2011).

22. Kleinloog, R., Mul, N. D., Post, J. A. \& Rinkel, G. J. E. Risk Factors for Intracranial Aneurysm Rupture : A systematic review. Neurosurgery 82, 431-440 (2018).

23. Liang, L., Steinman, D. A., Brina, O., Chnafa, C., Cancelliere, N. M. \& Pereira, V. M. Towards the Clinical utility of CFD for assessment of intracranial aneurysm rupture - a systematic review and novel parameter-ranking tool. Journal of NeuroInterventional Surgery 11, 153-158 (2019).

24. Xiang, J., Natarajan, S. K., Tremmel, M. et al. Hemodynamic-Morphologic Discriminants for Intracranial Aneurysm Rupture. Stroke 42, 144-152 (2011).

25. Miura, Y., Ishida, F., Umeda, Y. et al. Low Wall Shear Stress Is Independently Associated With the Rupture Status of Middle Cerebral Artery Aneurysms. Stroke 44, 519-521 (2013).

26. Geers, A. J., Larrabide, I., Radaelli, A. G. et al. Patient-specific computational hemodynamics of intracranial aneurysms from $3 \mathrm{D}$ rotational angiography and CT angiography: An in vivo reproducibility study. American Journal of Neuroradiology 32, 581-586 (2011).

27. Marzo, A., Singh, P., Larrabide, I. et al. Computational hemodynamics in cerebral aneurysms: The effects of modeled versus measured boundary conditions. Annals of Biomedical Engineering 39, 884-896 (2011).

28. Mulder, G., Marzo, A., Bogaerds, A. C. B. et al. Patient-Specific Modeling of Cerebral Blood Flow: Geometrical Variations in a $1 \mathrm{D}$ Model. Cardiovascular Engineering and Technology 2, 334-348 (2011).

29. Thomas, C., Nithiarasu, P. \& Bevan, R. The locally conservative Galerkin (LCG) method for solving the incompressible Navier Stokes equations. International Journal for Numerical Methods in Fluids 57, 1771-1792 (2008).

3o. Müller, L., Parés, C. \& Eleuterio, F. Well-balanced high-order numerical schemes for one-dimensional blood flow in vessels with varying mechanical properties. Journal of Computational Physics 242, 53-85 (2013)

31. Watanabe, S., Blanco, P. \& Raúl, F. Mathematical model of blood flow in an anatomically detailed arterial network of the arm. ESAIM: Mathematical Modelling and Numerical Analysis 47, 961-985 (2013).

32. Kroon, W., Huberts, W., Bosboom, M. \& van de Vosse, F. A numerical method of reduced complexity for simulating vascular hemodynamics using coupled oD lumped and $\mathrm{1} \mathrm{D}$ wave propagation models. Computational and Mathematical Methods in Medicine 2012, 1-10 (2012).

33. Azer, K. \& Peskin, C. S. A one-dimensional model of blood flow in arteries with friction and convection based on the Womersley velocity profile. Cardiovascular Engineering 7, 51-73 (2007).

34. Mynard, J. \& Valen-sendstad, K. A unified method for estimating pressure losses at vascular junctions. Int. J. Numer. Meth. Biomed. Engng. 1-23 (2015). 

PART

Non-invasive ICP assessment

using the ONSD 



\section{CHAPTER}

\section{The Influence of Discrepancies in Assessment Methodologies on the Optic Nerve Sheath Diameter}

Based on: Stevens, R.R.F., Gommer, E.D., Ariës, M., Ertl, M., Mess, W.H., Huberts, W. \& Delhaas, T. The Influence of Discrepancies in Assessment Methodologies on the Optic Nerve Sheath Diameter (submitted). 


\section{Abstract}

Reported cut-off values of the optic nerve sheath diameter (ONSD) for the diagnosis of elevated intracranial pressure (ICP) are inconsistent. This hampers ONSD as a possible non-invasive bedside monitoring tool for ICP. Since the influence of methodological differences on variations in cut-off values is unknown, we performed a narrative review to identify discrepancies in ONSD assessment methodologies and to investigate their effect on reported ONSD values. In a total of 63 eligible articles we could determine the applied ONSD assessment methodology. Reported ultrasound images either showed the optic nerve and its sheath as a dark region with hyperechoic striped band at its edges or as a single dark region surrounded by lighter retrobulbar fat. Four different ultrasound marker positions were used to delineate the optic nerve sheath, which resulted in different ONSD values and different sensitivities to changes in ICP. Based on our observations we recommend to place ultrasound markers at the outer edges of the hyperechoic striped bands or at the transitions from the single dark region to the hyperechoic retrobulbar fat because these locations yielded the highest sensitivity of ONSD measurements for increased ICP. 


\subsection{Introduction}

$\square_{\text {H }}^{\mathrm{E}}$ subarachnoid space, located between the optic nerve and the optic nerve sheath, contains cerebrospinal fluid (CSF). When the hydrostatic intracranial pressure (ICP) increases the optic nerve sheath can expand. Because this sheath expansion is considered to be a surrogate parameter for non-invasive ICP estimation $[1,2]$ the optic nerve sheath diameter (ONSD) is often measured in clinical practice. Although intra- and interobserver agreement of ONSD estimation is high within individual studies [3, 4], ONSD cut-off values for the diagnosis of elevated ICP (>15 $\mathrm{mmHg})$ vary between studies [5, 6] and thereby limit the application of ONSD assessment as a non-invasive surrogate marker for ICP monitoring. It is hence of utmost importance to identify and resolve sources of these variations in cut-off values. Although differences between patient populations studied might explain part of these variations, the role of discrepancies in ONSD assessment methodologies still needs to be determined. Methodological aspects, such as ultrasound machine settings, transducer placement, and ONSD delineation, probably differ between clinical centers and research groups and might hence contribute to the diversity in ONSD cut-off values.

In this study, we perform a narrative literature review in which we identify and categorize the reported ONSD assessment methodologies and investigate the effect of differences found on the measured ONSD values. Hereby, we focus on 1) the characteristic appearances of reported ultrasound images, 2) how the visible structures are interpreted with respect to the retrobulbar anatomy, 3) how these interpretations guide placement of the ultrasound markers used to calculate the ONSD, and 4) the relationship between ONSD values and assessment methodology.

\subsection{Materials and Methods}

\subsubsection{Eligibility criteria and search strategy}

In December 2019, a PubMed search was performed using the following search strategy: ("optic nerve sheath" AND "diameter" [MeSH Terms]) AND ("elevated icp intracranial pressure" [MeSH Terms]). Titles and abstracts of the search results were evaluated and articles were selected for full text review based on the following criteria: 1) articles were written in English, 2) ONSD was manually assessed from ultrasound images, and 3) articles did not involve fetus, animal or in vitro studies. Consecutive full text review was performed to select articles according to the following criteria: 4) articles did report ultrasound images, 5) reported ultrasound images were of such quality that the borders between darker and lighter regions could be distinguished, 6 ) ultrasound markers were visible within the images, and 
7) there was no ambiguity in reported ultrasound images with respect to patterns of lighter and darker regions. Criterion 5 and 6 were applied because our interest is in the specific characteristics of the ultrasound images, i.e. the lighter and darker regions of the images, the structural interpretation of these regions, and the corresponding ultrasound marker placement. Images of inferior quality would prevent us from extracting this information from the articles. We applied criterion 7 because articles with ambiguous images would prevent us from assigning them to one of the branches in the structured and quantitative approach that was applied to analyze the selected articles and will be described in Section 2.2.3.

\subsubsection{Optic nerve anatomy}

To facilitate referring to different retrobulbar structures in the remainder of the manuscript, we first describe the anatomy of the optic nerve and the optic nerve sheath (see Figure 2.1). The optic nerve runs from the eyeball to the chiasm and can be divided into four parts, i.e. an intra-ocular part ( $1 \mathrm{~mm}$ long), intra-orbital part (30 mm long), intracanalicular part (6-10 mm long), and intracranial part (10-16 mm long) [7]. Nerve fibers originate from the retinal ganglion cells and join at the optic disc, which is commonly referred to as "the blind spot". From here, the bundled unmyelinated optic nerve fibers run approximately $1 \mathrm{~mm}$ through the globe before penetrating the sclera through the lamina cribrosa. Once the optic nerve enters the intra-orbital space, it is surrounded by the optic nerve sheath. Besides the optic nerve and its sheath, the region immediately behind the globe also contains extraocular muscles and fat tissue. The optic nerve itself has a diameter of approximately $3 \mathrm{~mm}$, whereas the optic nerve sheath has a thickness of approximately $1 \mathrm{~mm}$ [8]. From in to out, the sheath consists of the pia mater, the subarachnoid space, the arachnoid mater, and the dura mater[9]. The pia mater and the arachnoid mater have a thickness of $0.09-0.15 \mathrm{~mm}[10,11]$, whereas the subarachnoid space and the dura have a thickness of $0.1-0.29 \mathrm{~mm}$ and $0.3-0.5 \mathrm{~mm}[8,10,11]$, respectively. The subarachnoid space features a complex structure formed by trabeculae, septa, and pillars immersed in the cerebral spinal fluid. The composition of these structures within the subarachnoid space changes along the nerve[12]. The anterior part of the subarachnoid space mainly contains trabeculae, which are 5-7 $\mu \mathrm{m}$ in diameter. The midsection of the sheath displays both septa that divide this space into small communicating chambers, as well as pillars with a diameter of $10-30 \mu \mathrm{m}[12]$. In the posterior part, where the sheath crosses the optic canal, both pillars and trabeculae are present. 


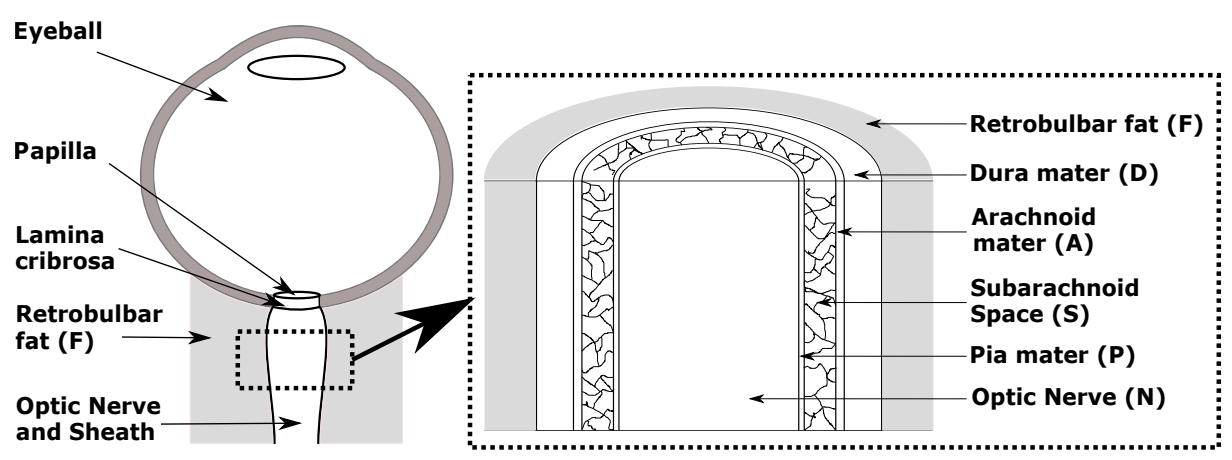

Figure 2.1: Left: Schematic overview of the anatomy of the eyeball and optic nerve. The nerve and its sheath are surrounded by retrobulbar fat tissue. Right: A detailed drawing of the different layers of the optic nerve sheath. The nerve is surrounded by the pia mater, subarachnoid space, arachnoid mater, and the dura mater.

\subsubsection{Structured and quantitative approach}

To investigate the effect of different methodologies on ONSD values we assigned a "code" to the ONSD assessment methodology used in each reviewed article. This classification was based on the criteria discussed below.

\section{Characteristics of retrobulbar ultrasound images}

Two groups of retrobulbar ultrasound images with different patterns of echoic characteristics were found in the reviewed articles. Both groups clearly showed a dark (hypoechoic) circular structure representing the eyeball. In addition, lighter (hyperechoic) structures were present behind the globe representing the retrobulbar fat and ocular muscles. The optic nerve was represented by a linear dark structure perpendicular to the globe. However, whereas one group of images only showed a single dark linear structure surrounded by lighter retrobulbar fat behind the eyeball, the other group displayed two lighter (hyperechoic) striped "bands" within this dark region (Figure 2.2, top panel). In order to denote these echoic appearances of the reported ultrasound images in our quantitative analysis, we labeled images with striped bands "Category A", and images without these dark bands "Category B" (Figure 2.2, mid panel). 


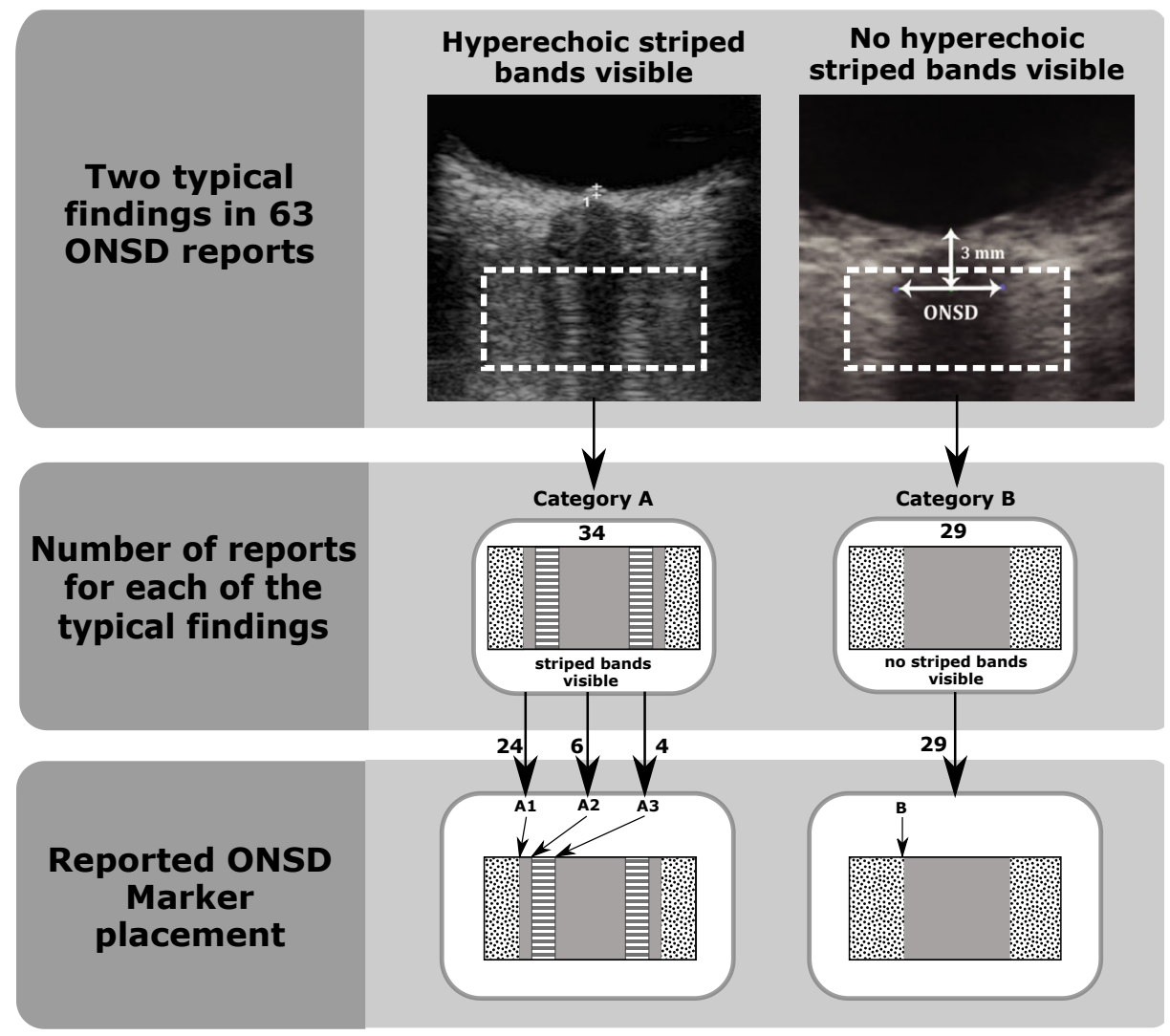

Figure 2.2: Structured and Quantitative approach used to label the optic nerve sheath diameter (ONSD) methodologies based on the ultrasound image characteristics and marker placement. Top: Two types of typical findings in ONSD reports, i.e. ultrasound images show hyperechoic striped bands in between the hyperechoic retrobulbar fat, and ultrasound images show no hyperechoic striped bands. Ultrasound images obtained from $[13,14]$. Middle: Characterization of the typical findings in to Category A and Category B. Schematic representations of the ultrasound images are depicted in the figures given within each category. Bottom: Standardized marker positions based on the transitions from lighter to darker regions. 


\section{Marker placement for sheath assessment}

To quantify placement of ultrasound markers used to delineate the optic nerve sheath, possible locations of ultrasound markers were labeled. In the presence of hyperechoic striped bands, possible marker positions from outside to inside were located at: A1) transition from hyperechoic retrobulbar fat region to hypoechoic line, A2) transition from hypoechoic line to hyperechoic striped band, A3) transition from hyperechoic striped band to hypoechoic region. In images without striped bands, marker placement is only possible at the transition from the hyperechoic region to the hypoechoic region and this location was denoted with letter $\mathrm{B}$.

\section{Marker placement for depth assessment}

Three possible positions for ultrasound depth markers were defined and used to label the depth marker placement within the articles (Figure 2.3): 1) location where imaginary nerve midline intersects contour of the retina, 2) hyperechoic reflection corresponding to the lamina cribrosa, and 3) top of the dark linear structure corresponding to the optic nerve. In case no marker was placed within the reported image, the article was labeled with "marker position not presented". In addition, the terminology used to describe the chosen anatomical landmark was extracted. If no landmark was specified it was denoted with "not reported".

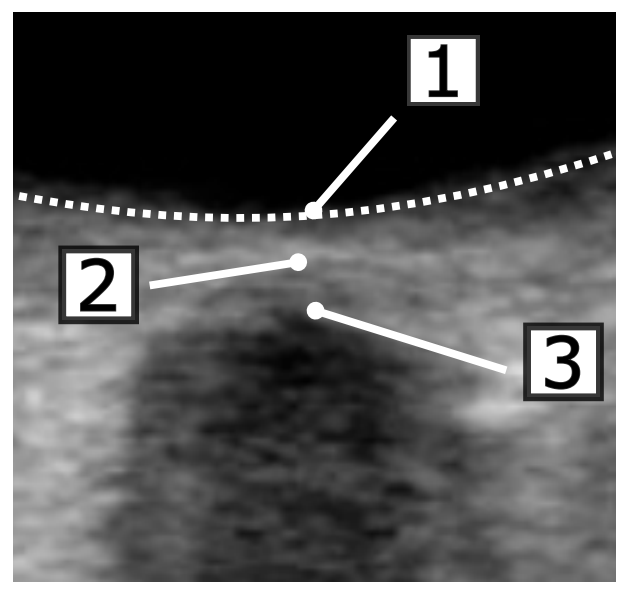

Figure 2.3: Definition of the three depth marker positions found in literature: 1) at the level of the retina, 2) at the level of the lamina cribrosa, and 3) at the top of the hypoechoic (black) optic nerve. The white dotted line denotes the retina. 


\subsubsection{Structural interpretation}

We also classified how echoic properties of the optic nerve and its sheath were described and how structures as seen on the ultrasound images were interpreted. Moreover, we examined if the interpretation of the ultrasound images was similar across studies.

\subsubsection{Ultrasound settings}

Transducer frequency, imaging plane (transverse or sagittal), and mechanical index (MI) were extracted from the articles to investigate whether image acquisition characteristics were related to the ONSD values.

\subsubsection{Influence of methodology on measured ONSD values}

Clinical relevance of an ONSD methodology depends on its ability to detect changes in ICP. Therefore, we assessed for each of the different ONSD measurement methodologies how the corresponding ONSD values changed with increasing ICP. ONSD values for subjects with normal (low) and with pathologically elevated (high) ICPs were extracted from the selected articles. It is to be noted that low ICP subjects were reported in the reviewed articles as healthy persons $(\mathrm{H})$, as age-matched controls $(\mathrm{C})$, or as patients with normal ICP $\left(\mathrm{ICP}_{\text {norm }}\right)$. Extracted ONSD values were separated based on the assessment methodology described in Section 2.2.3, i.e. Al, $\mathrm{A}_{2}, \mathrm{~A}_{3}$, and $\mathrm{B}$ (see Figure 2.2). ONSD values obtained with the different methods were compared within each of the four different subject groups. Subsequently, differences in ONSD values between these subject groups were investigated for each of the four methodologies to assess their sensitivity for ICP elevation. We also investigated the influence of variations in ultrasound depth marker placement on the ONSD values.

\subsection{Results}

\subsubsection{Search results}

We identified 191 citations based on our PubMed search criteria. After screening the abstracts of these studies, 67 studies were excluded because they: involved animal, fetus, or in-vitro studies $(n=8)$, assessed ONSD from CT/MRI images $(n=21)$, were comments on other articles ( $n=23)$, were not written in English $(n=8)$, were not available $(n=3)$, or other $(n=4)$. The remaining 124 articles were selected for full text review. From this set an additional 30 articles were excluded because they: assessed ONSD from MRI $(n=1)$, were comments on other articles $(n=1)$, were not written 
in English $(n=1)$, were not available $(n=1)$, were review articles without an ONSD methodology section $(n=17)$, showed unclear exemplary ultrasound images $(n=3)$, showed no ultrasound markers within the image $(n=2)$, showed ultrasound images with conflicting characteristic appearance $(n=2)$, or other $(n=2)$. After omitting all papers that did not present any ultrasound image at all $(n=31)$, a total of 63 articles remained for analysis in our review $[1,2,5,13-72]$.

\subsubsection{Structured and quantitative approach}

Image characteristics: The number of articles corresponding to each of the typical ultrasound findings are indicated in the middle of Figure 2.2. Approximately half $(n=34)$ of the articles showed images corresponding to Category A, i.e. depicting hyperechoic striped bands within the hypoechoic region between the retrobulbar fat. The other half $(n=29)$ reported images corresponding to Category B displaying no striped bands in the dark region between the retrobulbar fat.

Marker placement for sheath assessment: The marker position for the sheath delineation (A1, A2, $A_{3}$, and $B$ ) was determined in all 63 articles (Figure 2.2). In the majority of articles within Category A, the marker was placed at the most outer transition from hyperechoic to the hypoechoic region, i.e. Location A1. For the articles showing images corresponding to Category B the marker was by definition set at position B.

Marker placement for depth assessment: A total of 60 out of 63 articles reported the depth at which the ONSD was determined. Although all of these 60 articles reported that the ONSD was determined $3 \mathrm{~mm}$ posterior to an anatomical landmark, we encountered three different anatomical landmark positions for which 9 different names were used with no clear distinction between them.

\subsubsection{Structural interpretation}

Detailed perceptions about the echogenic properties of the different retrobulbar structures and the corresponding structural interpretations of the ultrasound images were found in eight articles. Although all eight articles stated that the optic nerve does not reflect ultrasound waves and appears as a dark structure radiating away from the globe, the opinions on the echoic properties of the different layers of the optic nerve sheath differed. Based on the latter differences, the structural interpretations could be divided into two groups (see Figure 2.4).

In the first interpretation, reported in six articles [15, 21, 25, 41, 47, 64], it was assumed that the pia mater and the dura mater do not reflect ultrasound waves and that the subarachnoid space and the retrobulbar fat are hyperechoic. Hence, the pia mater was thought to appear as a dark structure fused with the also dark optic nerve, the subarachnoid space was appointed to the two hyperechoic striped bands, and the 


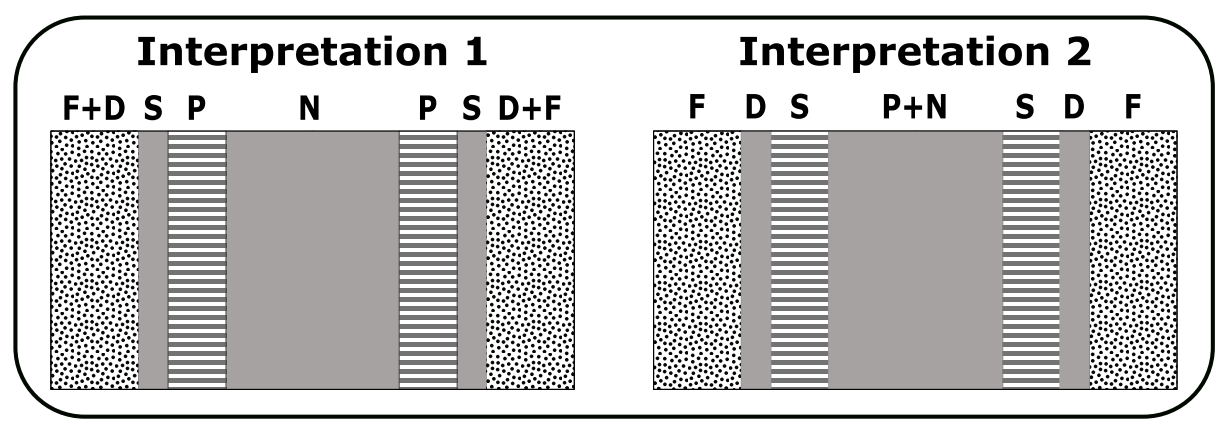

Figure 2.4: The two different structural interpretations of ultrasound images found in literature. The darker and lighter regions within the schematic depictions of the ultrasound images are labeled with optic nerve $(\mathrm{N})$, pia mater $(\mathrm{P})$, subarachnoid space $(\mathrm{S})$, dura mater $(\mathrm{D})$, and retrobulbar fat tissue $(\mathrm{F})$ following the description of the echoic fingerprint of the optic nerve and its sheath as described in literature.

dura mater was thought to appear as a dark line in-between the white retrobulbar fat and the hyperechoic striped bands.

In the second interpretation, described in two articles[1, 67], it was assumed that the subarachnoid space does not reflect ultrasound waves and that the pia mater, the dura mater, and the retrobulbar fat are hyperechoic. Hence, it was stated that the pia mater corresponds with the hyperechoic striped bands present within the dark region and that the subarachnoid space is represented by the thin hypoechoic lines in between the striped bands and the surrounding white tissue. Moreover, the dura mater and retrobulbar fat are thought to cause acoustic reflections and therefore appear as hyperechoic structures which cannot be distinguished from each other.

\subsubsection{Ultrasound settings}

\section{Transducer Frequency}

A total of 60 out of 63 articles did report the transducers used. Although, all transducers were linear, their frequencies ranged from $3 \mathrm{MHz}$ up to $25 \mathrm{MHz}$, with $7 \cdot 5 \mathrm{MHz}$ being the most used frequency $(n=13)$.

\section{Mechanical index}

MI settings were reported in 19 out of 63 articles. Values $<0.3$ were reported in 13 articles, the "As Low As Reasonable Achievable" (ALARA) principle was reported in five articles, whereas one article reported to have used an MI that was "low". 


\section{Imaging plane}

The imaging plane was reported in 49 out of 63 articles. In the majority of cases $(n=40)$ a transverse plane was applied, either solely $(n=37)$ or in combination with the sagittal plane $(n=3)$. Nine studies reported on solely using the sagittal plane.

\section{Relation between ultrasound settings and image characteristics}

Distributions of transducer frequencies and imaging planes were similar for articles appointed to Category A and articles appointed to Category B indicating that the presence of hyperechoic striped bands is not related to transducer frequency or image plane used.

\subsubsection{Influence of methodology on the Optic Nerve Sheath Diameter}

ONSD values for the four subject groups are plotted for each assessment method $\left(\mathrm{A}_{1}, \mathrm{~A}_{2}, \mathrm{~A}_{3}\right.$, and $\left.\mathrm{B}\right)$ in Figure 2.5. In addition, mean ONSD values per assessment method for each of the four subject groups are given in Table 2.1. Assuming that both the healthy group and the age-matched control group have normal ICPs, we pooled the ONSD values of both groups because only a few observations were available for the healthy population. Moreover, Table 2.1. shows mean differences between ONSD values of the various subject groups. From Figure 2.5 and Table 2.1. the following trend can be observed: averaged over the four subject groups, ONSD values determined with Method A1 are o.67 (o.2-1.2) mm larger than ONSD values determined with Method A2. In turn, these A2 derived ONSD values are 1.43 (o.7-1.9) mm larger than the ONSD values obtained with A3. The ONSD values determined with Method B are similar to the values obtained with Method A2. It is evident from Table 2.1 that the difference in ONSD values between patients with $\mathrm{ICP}_{\text {norm }}(n=21)$ and patients with $\mathrm{ICP}_{\text {high }}(n=31)$ is on average at least three times larger for Methods A1, A2, and B in comparison with Method A3, i.e. 0.9, 1.5, 1.5 and $0.3 \mathrm{~mm}$, respectively. We did not find differences in ONSD values due to variations in depth marker positioning.

\subsection{Discussion}

We performed a narrative literature review to identify possible methodological differences in ONSD determination by means of ultrasound and to evaluate whether such differences affect the ONSD values within and between groups of patients with normal and pathologically elevated ICP. Hereto, we used a structured and quantitative approach to label the different ONSD methods and to link the reported ONSD values to these methods. 


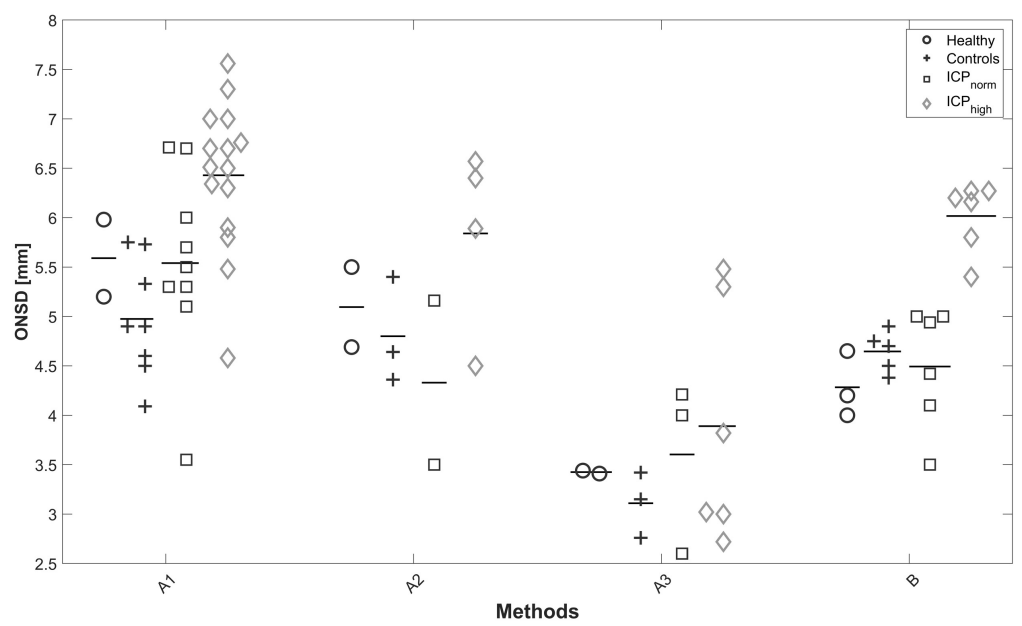

Figure 2.5: ONSD values for healthy people, age-matched controls, patients with $\mathrm{ICP}_{\text {norm }}$, and $\mathrm{ICP}_{\text {high }}$ reported by the reviewed articles. The ONSD values are classified into assessment Methodologies Al, $\mathrm{A}_{2}, \mathrm{~A}_{3}$, or $\mathrm{B}$. The lines denote the mean values for each assessment methodology.

\subsubsection{Major Findings}

We identified four different ultrasound marker positions used to measure the ONSD, which resulted in varying ONSD values. Moreover, not all marker positions were equally sensitive to changes in ICP between subjects with elevated and those with normal ICP. Three of the marker positions were located within images with hyperechoic striped bands (i.e. A1, A2, and $A_{3}$ ), whereas the other position was pinpointed in images without hyperechoic bands (i.e. B). The three marker positions identified within images with striped bands, resulted in different ONSD values. Placing the markers at the transition from the hyperechoic retrobulbar fat to the dark region behind the globe in both image types (respectively, A1 and B), did on average not yield similar ONSD values. However, ONSD values obtained when ultrasound markers were placed at the outer edges of the striped bands (A2) were comparable to the ones derived with marker location $\mathrm{B}$.

Clinically most interesting is the ability of the different methods to distinguish between patients with $\mathrm{ICP}_{\text {norm }}$ and patients with $\mathrm{ICP}_{h i g h}$. Our results showed that placement of the markers at the most inner edges of the hyperechoic striped bands (A3) was far less sensitive to changes in ICP than the other marker positions.

Besides the four different ultrasound marker positions within the two types of ultrasound images, we also encountered poor reporting of ONSD methodologies 
Table 2.1: Upper part: Mean ONSDs categorized in study population (Healthy and controls, Normal ICP and Elevated ICP) and methodologies used to determine the ONSDs ( $\mathrm{A}_{1}, \mathrm{~A}_{2}, \mathrm{~A}_{3}$, and $\mathrm{B}$ ). The number of ONSD values are shown between brackets. Lower part: The difference in ONSDs between study populations for each of the methodologies (bottom).

\begin{tabular}{lllll}
\hline \multirow{2}{*}{ Population } & \multicolumn{4}{c}{ Methodological Categories } \\
\cline { 2 - 5 } & $\begin{array}{l}\text { A1 } \\
(n=10 ; 10 ; 15)\end{array}$ & $\begin{array}{l}\text { A2 } \\
(n=6 ; 2 ; 4)\end{array}$ & $\begin{array}{l}\text { A3 } \\
(n=5 ; 3 ; 6)\end{array}$ & $\begin{array}{l}\text { B } \\
(n=8 ; 6 ; 6)\end{array}$ \\
\hline $\begin{array}{l}\text { Healthy and controls } \\
(\mathrm{H}+\mathrm{C})\end{array}$ & 5.1 & 4.9 & 3.2 & 4.5 \\
$\begin{array}{l}\text { Normal ICP } \\
\left(\mathrm{ICP} \mathrm{n}_{\text {norm }}\right)\end{array}$ & 5.5 & 4.3 & 3.6 & 4.5 \\
$\begin{array}{l}\text { Elevated ICP } \\
\left(\mathrm{ICP} \mathrm{P}_{\text {high }}\right)\end{array}$ & 6.4 & 5.8 & 3.9 & 6.0 \\
\hline $\mathrm{ICP}_{\text {norm }}-(\mathrm{H}+\mathrm{C}) \mid$ & 0.4 & 0.6 & 0.4 & 0 \\
$\left|\mathrm{ICP}_{\text {high }}-(\mathrm{H}+\mathrm{C})\right|$ & 1.3 & 0.9 & 0.7 & 1.5 \\
$\mid \mathrm{ICP}_{\text {high }}-\mathrm{ICP}$ norm & 0.9 & 1.5 & 0.3 & 1.5 \\
\hline
\end{tabular}

within a large portion of the analyzed articles as well as discrepancies in ONSD assessment methodologies (vide infra). Moreover, a detailed interpretation of the echoic signature of the optic nerve sheath was only reported in 8 out of the 63 articles. Within these eight articles two different, very conflicting interpretations of the echoic signature were found.

Anatomical landmarks used to calculate the ONSD assessment depth also varied between studies, resulting in different depth marker positions. Moreover, the terminology used to describe these landmarks was inconsistent and identical anatomical terms were sometimes used to describe different landmarks. Ultrasound transducer frequencies were reported in almost every article and their range varied widely. Reported imaging planes also varied between studies, with studies using only a transverse plane or both the transverse and sagittal plane, whereas one third of the articles did not report the imaging plane at all.

\subsubsection{Considerations}

The lack of agreement on the echoic signature of the optic nerve sheath is most likely the cause for the variation in ultrasound marker placement. Although there is no ambiguity that the optic nerve is represented by the hypoechoic longitudinal structure, the appearance of the pia mater, the subarachnoid space, and the dura mater seems to be much less agreed upon. Studies of Topcuoglu et al. [67] and Geeraerts et al. [1] dedicate the hyperechoic striped bands to the pia mater. Pichamuthu et al. [6], on the other hand, interpret the striped bands as being both the pia and 
dura mater, with the subarachnoid space only appearing in the middle of these bands in patients with elevated ICP. Others assume that these bands represent the subarachnoid space $[15,19,25,64]$ which reflects ultrasound waves because of the complex structure of trabeculae and pillars[73]. A study of Steinborn et al. [64] even describes the formation of a cyst like structure within the subarachnoid space in patients with elevated ICP due to the stretching of the trabeculae. Another study reports that these striped bands are only visible in patients with elevated intracranial pressure[74], indicating that these bands represent the subarachnoid space and that these bands only become apparent on ultrasound images when the subarachnoid space is enlarged.

Besides the different interpretations of the echoic fingerprint of the optic nerve sheath, the duality in echoic characteristic patterns of the ultrasound images (Category A and Category B) needs to be considered. Although our data did not show an association between appearance of hyperechoic striped bands and ultrasound frequency or imaging plane, a recent study from Pichamuthu et al. [6] points out that ultrasound images might be dependent on the transducer frequency used because high frequency transducers allow imaging of smaller structures in comparison with lower frequency probes. Moreover, the usage of higher frequency probes makes the ONSD assessment possibly less susceptible to edge artefacts because the true outlines of the optic nerve sheath are more clearly visible[6]. It cannot be excluded that patient characteristics such as gender or age, also play a role whether hyperechoic striped bands appear on retrobulbar ultrasound images.

The marker placement used to determine the assessment depth of $3 \mathrm{~mm}$ also appears to be an enigma as becomes apparent by the variation in terms used to annotate a specific anatomical landmark and the use of the same term for different anatomical landmarks. However, these discrepancies did not affect ONSD values, most likely, because the distance between the different anatomical landmarks used is less than $1 \mathrm{~mm}$ resulting in comparable ONSD values. Nevertheless, the usage of a singular landmark and corresponding terminology could avoid confusion.

For all groups, i.e. healthy subjects and age-matched controls as well as patients

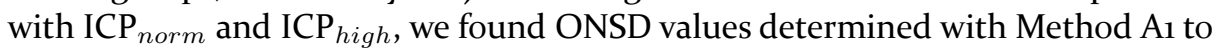
be larger than values determined with $\mathrm{A} 2$, which in turn were larger than values obtained with Methodology A3. This finding is trivial because the markers for Position 1, 2, and 3 are located from outside to inside on the ultrasound image. However, the difference in ONSD values between Methods A2 and A3 was twice as large as the difference between ONSD values assessed with Methods A1 and A2. The difference in ONSD values between healthy/controls and patients with $\mathrm{ICP}_{\text {norm }}$ and $\mathrm{ICP}_{\text {high }}$ showed that Method $\mathrm{A}_{3}$ was the only method that did not allow a clear distinction between the high and low ICP groups, making Method A3 clinically not useful. Moreover, the small difference in ONSD values between $\mathrm{ICP}_{\text {norm }}$ and $\mathrm{ICP}_{\text {high }}$ for Method $\mathrm{A}_{3}$ is a clear indication that this method does not 
measure the subarachnoid space since the subarachnoid space is the only structure that expands when ICP increases. In addition, ONSD values determined with $\mathrm{A}_{3}$ were mostly in the order of $3 \mathrm{~mm}$ which is approximately the diameter of the optic nerve itself. Therefore, marker position A3 corresponds with the outline of the optic nerve. This, in combination with the fact that ONSD values assessed with position A2 do become significantly larger in patient with $\mathrm{ICP}_{h i g h}$, is a clear indication that marker position Az incorporates the subarachnoid space. This makes it very likely that marker position A2 corresponds to the outer edge of the subarachnoid space which appears as the hyperechoic striped bands. Consequently, marker placements as described in Method A1 and B both correspond to the outer edges of the dura mater.

Placing the markers at the positions of Method A2 and B yields similar ONSD values, and both methods are found to be equally sensitive to changes in ICP. In clinical practice, it does therefore not matter whether either ultrasound images with hyperechoic bands in combination with Method A2, or ultrasound images without hyperechoic bands in combination with Method B, are used for ONSD assessment.

\subsubsection{Limitations}

One limitation of our study is that we did not consider the methodological descriptions of ultrasound marker placement, but only focused on the exemplary ultrasound images. The primary reason for this constraint was that researchers often provide a description of the ultrasound marker placement that is inconclusive or cite (multiple) other studies in which the methodological approach is inconclusive, making it impossible to categorize the methodology that was actually used based on their description. This observation further highlights our conclusions regarding the urgent need for ONSD standardization and improved documentation.

Another limitation is that, although articles were categorized based on their reported exemplary ultrasound image, ONSD assessment might have been performed differently. Moreover, in some cases it was hard to differentiate whether ultrasound marker placement was at position A1 or A2 because the dark lines between hyperechoic striped bands and hyperechoic retrobulbar fat were often very narrow. Because only 63 articles could be included for our analysis, we had limited number of ONSD values for each of the four groups. 


\subsubsection{Recommendations}

Our results showed not only a large variety in ONSD assessment methodology, but also that ONSD values depend on the methodologies used. This variety in measurement approaches could be an explanation for the discrepancies in the threshold values for elevated ICP reported in literature. Based on our findings we provide the following crucial instructions for reliable and apparent ONSD measurements:

- 1) Place ultrasound markers at the outer edges of the hyperechoic striped bands or at the transitions from the single dark region to the hyperechoic retrobulbar fat.

-2) Use the papilla as a reference point for the $3 \mathrm{~mm}$ depth assessment.

- 3) Mechanical Index (MI) has to be kept at values smaller than or equal to 0.3 to ensure patient safety. The TI index, on the contrary is not relevant for this examination.

- 4) Although adequate ONSD measurements can be performed with almost any linear transducer available, we suggest the usage of linear transducers with the highest possible frequency to optimize resolution.

\subsection{Conclusion}

In this study, we performed a literature review on ONSD methodologies and examined their effect on ONSD values. We found discrepancies in ultrasound machine settings, characteristic appearances of ultrasound images, structural interpretation of these images, and ultrasound marker placement. More importantly, our results showed that ONSD values depend on the methodologies used. Based on our findings we propose a set of recommendations for ultrasound imaging of the optic nerve sheath, choice of anatomical landmark used to determine ONSD assessment depth, as well as choice of marker positions that annotate the optic nerve sheath. These recommendations will decrease the variation in ONSD values due to assessment discrepancies and possibly improve the accuracy of non-invasive bedside ICP monitoring. 


\section{References}

1. Geeraerts, T., Merceron, S., Benhamou, D. et al. Non-invasive assessment of intracranial pressure using ocular sonography in neurocritical care patients. eng. Intensive Care Medicine 34, 2062-2067 (2008).

2. Toscano, M., Spadetta, G., Pulitano, P. et al. Optic Nerve Sheath Diameter Ultrasound Evaluation in Intensive Care Unit: Possible Role and Clinical Aspects in Neurological Critical Patients' Daily Monitoring. BioMed Research International 2017, 1-7 (2017).

3. Bäuerle, J., Lochner, P., Kaps, M. \& Nedelmann, M. Intra- and Interobsever Reliability of Sonographic Assessment of the Optic Nerve Sheath Diameter in Healthy Adults. Journal of Neuroimaging 22, 42-45 (2012).

4. Lochner, P., Coppo, L., Cantello, R. et al. Intra- and interobserver reliability of transorbital sonographic assessment of the optic nerve sheath diameter and optic nerve diameter in healthy adults. Journal of Ultrasound 19, 41-45 (2016).

5. Wang, L.-J. J., Yao, Y., Feng, L.-S. S. et al. Noninvasive and quantitative intracranial pressure estimation using ultrasonographic measurement of optic nerve sheath diameter. eng. Scientific Reports 7, 1-7 (2017).

6. Pichamuthu, K. K. \& Prithishkumar, I. J. Appearance of the Optic Nerve Sheath Diameter (ONSD) using Higher Frequency Linear Probes in Detection and Monitoring of Raised Intracranial Pressures-A Cadaveric Study. Journal of Clinical and Diagnostic Research, 10-13 (2019).

7. Remington, L. A. \& Goodwin, D. Clinical Anatomy of the Visual System 2nd Edition 2nd ed. (Butterworth-Heinemann, 2004).

8. Liu, D. \& Kahn, M. Measurement and Relationship of Subarachnoid Pressure of the Optic Nerve to Intracranial Pressures in Fresh Cadavers. American Journal of Ophthalmology 116, 548-556 (1993).

9. Helmke, K. \& Hansen, H. C. Fundamentals of transorbital sonographic evaluation of optic nerve sheath expansion under intracranial hypertension. II. Patient study. eng. Pediatric Radiology 26, 706-710 (1996).

10. Pache, M. \& Meyer, P. Morphological changes of the retrobulbar optic nerve and its meningeal sheaths in glaucoma. Ophthalmologica 220, 393-396 (2006).

11. Lang, J. Clinical Anatomy of the Head 1st (eds Wilson, R. \& Winstanley, D.) (Springer-Verlag, Würzburg, 1983).

12. Killer, H. E., Laeng, H. R., Flammer, J. \& Groscurth, P. Architecture of arachnoid trabeculae, pillars, and septa in the subarachnoid space of the human optic nerve: anatomy and clinical considerations. Br J Ophthalmol 87, 777-781 (2003).

13. Lochner, P., Brigo, F., Zedde, M. L. et al. Feasibility and usefulness of ultrasonography in idiopathic intracranial hypertension or secondary intracranial hypertension. eng. BMC neurology 16, 85 (2016).

14. Dinsmore, M., Han, J. S., Fisher, J. A., Chan, V. W. S. \& Venkatraghavan, L. Effects of acute controlled changes in end-tidal carbon dioxide on the diameter of the optic nerve sheath: a transorbital ultrasonographic study in healthy volunteers. eng. Anaesthesia 72, 618-623 (2017).

15. Bäuerle, J., Nedelmann, M., Bauerle, J., Nedelmann, M., Bäuerle, J. \& Nedelmann, M. Sonographic assessment of the optic nerve sheath in idiopathic intracranial hypertension. eng. Journal of Neurology 258, 2014-2019 (2011). 
16. Betcher, J., Becker, T. K., Stoyanoff, P., Cranford, J. \& Theyyunni, N. Military trainees can accurately measure optic nerve sheath diameter after a brief training session. eng. Military Medical Research 5, 42 (2018).

17. Bergmann, K. R., Milner, D. M., Voulgaropoulos, C., Cutler, G. J. \& Kharbanda, A. B. Optic Nerve Sheath Diameter Measurement During Diabetic Ketoacidosis: A Pilot Study. eng. The western journal of emergency medicine 17, 531-41 (2016).

18. Bolesch, S., von Wegner, F., Senft, C. \& Lorenz, M. W. Transcranial ultrasound to detect elevated intracranial pressure: Comparison of septum pellucidum undulations and optic nerve sheath diameter. eng. Ultrasound in Medicine and Biology 41, 1233-1240 (2015).

19. Chen, Q., Chen, W., Wang, M. et al. High-resolution transbulbar ultrasonography helping differentiate intracranial hypertension in bilateral optic disc oedema patients. eng. Acta ophthalmologica 95, e481-e485 (2017).

2o. Choi, S.-H. H., Min, K.-T. T., Park, E.-K. K., Kim, M.-S. S., Jung, J.-H. H. \& Kim, H. Ultrasonography of the optic nerve sheath to assess intracranial pressure changes after ventriculo-peritoneal shunt surgery in children with hydrocephalus: A prospective observational study. eng. Anaesthesia 7o, 1268-1273 (2015).

21. Del Saz-Saucedo, P., Redondo-Gonzalez, O., Mateu-Mateu, A., Huertas-Arroyo, R., Garcia-Ruiz, R. \& Botia-Paniagua, E. Sonographic assessment of the optic nerve sheath diameter in the diagnosis of idiopathic intracranial hypertension. eng. Journal of the Neurological Sciences 361, 122-127 (2016).

22. Ballantyne, J., Hollman, A. S., Hamilton, R. et al. Transorbital optic nerve sheath ultrasonography in normal children. eng. Clinical Radiology 54, 740-742 (1999).

23. Driessen, C., Bannink, N., Lequin, M. et al. Are ultrasonography measurements of optic nerve sheath diameter an alternative to funduscopy in children with syndromic craniosynostosis? Clinical article. eng. Journal of Neurosurgery: Pediatrics 8, 329-334 (2011).

24. Driessen, C., Van Veelen, M.-L. L. C. L. C., Lequin, M., Joosten, K. F. M. \& Mathijssen, I. M. J. Nocturnal ultrasound measurements of optic nerve sheath diameter correlate with intracranial pressure in children with craniosynostosis. eng. Plastic and Reconstructive Surgery 130, 448-451 (2012).

25. Ertl, M., Barinka, F., Torka, E. et al. Ocular Color-Coded Sonography - A Promising Tool for Neurologists and Intensive Care Physicians Die okuläre Farbduplexsonografie - Ungenutztes Potential für Neurologen und Intensivmediziner. Ultraschall in Med 35, 422-431 (2014).

26. Fagenholz, P. J., Gutman, J. A., Murray, A. F., Noble, V. E., Camargo, C. A. J. \& Harris, N. S. Evidence for increased intracranial pressure in high altitude pulmonary edema. eng. High altitude medicine E biology 8, 331-336 (2007).

27. Gao, Y., Li, Q., Wu, C., Liu, S. \& Zhang, M. Diagnostic and prognostic value of the optic nerve sheath diameter with respect to the intracranial pressure and neurological outcome of patients following hemicraniectomy. BMC Neurology 18, 1-7 (2018).

28. Geeraerts, T., Launey, Y., Martin, L. et al. Ultrasonography of the optic nerve sheath may be useful for detecting raised intracranial pressure after severe brain injury. eng. Intensive Care Medicine 33, 1704-1711 (2007).

29. Goel, R. S., Goyal, N. K., Dharap, S. B., Kumar, M. \& Gore, M. A. Utility of optic nerve ultrasonography in head injury. eng. Injury 39, 519-524 (2008).

3o. Hall, M. K., Spiro, D. M., Sabbaj, A., Moore, C. L., Hopkins, K. L. \& Meckler, G. D. Bedside optic nerve sheath diameter ultrasound for the evaluation of suspected pediatric ventriculoperitoneal shunt failure in the emergency department. eng. Child's nervous system : ChNS : official journal of the International Society for Pediatric Neurosurgery 29, 2275-2280 (2013). 
31. Hansen, G., Sellers, E. A., Beer, D. L., Vallance, J. K. \& Clark, I. Optic Nerve Sheath Diameter Ultrasonography in Pediatric Patients with Diabetic Ketoacidosis. Canadian Journal of Diabetes 40, 126-130 (2016).

32. Hassen, G. W., Bruck, I., Donahue, J. et al. Accuracy of optic nerve sheath diameter measurement by emergency physicians using bedside ultrasound. eng. Journal of Emergency Medicine 48, 450457 (2015).

33. Hassen, G. W., Nazeer, O., Manizate, F., Patel, N. \& Kalantari, H. The role of bedside ultrasound in pretherapeutic and posttherapeutic lumbar puncture in patient with idiopathic intracranial hypertension. eng. American Journal of Emergency Medicine 32, 1298.e3-1298.e4 (2014).

34. Helmke, K. \& Hansen, H. C. Fundamentals of transorbital sonographic evaluation of optic nerve sheath expansion under intracranial hypertension. I. Experimental study. eng. Pediatric Radiology 26, 706-710 (1996).

35. Jeon, J. P., Lee, S. U., Kim, S.-E. E. et al. Correlation of optic nerve sheath diameter with directly measured intracranial pressure in Korean adults using bedside ultrasonography. eng. PloS one 12, eo183170 (2017).

36. Jun, I.-J. J., Kim, M., Lee, J. et al. Effect of mannitol on ultrasonographically measured optic nerve sheath diameter as a surrogate for intracranial pressure during robot-assisted laparoscopic prostatectomy with pneumoperitoneum and the trendelenburg position. eng. Journal of Endourology 32, 608-613 (2018).

37. Kazdal, H., Kanat, A., Findik, H. et al. Transorbital Ultrasonographic Measurement of Optic Nerve Sheath Diameter for Intracranial Midline Shift in Patients with Head Trauma. eng. World Neurosurgery 85, 292-297 (2016).

38. Keyes, L. E., Paterson, R., Boatright, D., Browne, V., Leadbetter, G. \& Hackett, P. Optic Nerve Sheath Diameter and Acute Mountain Sickness. eng. Wilderness E Environmental Medicine 24, 105-111 (2013).

39. Kim, M. S., Bai, S. J., Lee, J. R., Choi, Y. D., Kim, Y. J. \& Choi, S. H. Increase in intracranial pressure during carbon dioxide pneumoperitoneum with steep trendelenburg positioning proven by ultrasonographic measurement of optic nerve sheath diameter. Journal of Endourology $\mathbf{2 8 ,}$ 801-806 (2014).

40. Kim, M.-S. S., Yoon, K. B., Yoon, D. M. \& Kim, D.-H. H. Effect of cervical sympathetic block on optic nerve sheath diameter measured by ultrasonography. eng. Ultrasound in Medicine and Biology 41, 1599-1604 (2015).

41. Kishk, N. A., Ebraheim, A. M., Ashour, A. S., Badr, N. M. \& Eshra, M. A. Optic nerve sonographic examination to predict raised intracranial pressure in idiopathic intracranial hypertension: The cut-off points. eng. The neuroradiology journal 31, 490-495 (2018).

42. Kitano, T., Nezu, T., Mukai, T., Uemura, J., Wada, Y. \& Yagita, Y. A Case of Hypertensive Encephalopathy with Enlarged Optic Nerve Sheath Measured by Transorbital Sonography. eng. Journal of Stroke and Cerebrovascular Diseases 26, e20-e21 (2017).

43. Komut, E., Kozaci, N., Sonmez, B. M. et al. Bedside sonographic measurement of optic nerve sheath diameter as a predictor of intracranial pressure in ED. eng. American Journal of Emergency Medicine 34, 963-967 (2016).

44. Beare, N. A. V., Kampondeni, S., Glover, S. J. et al. Detection of raised intracranial pressure by ultrasound measurement of optic nerve sheath diameter in African children. eng. Tropical Medicine E International Health 13, 1400-1404 (2008). 
45. Le, A., Hoehn, M. E., Smith, M. E., Spentzas, T., Schlappy, D. \& Pershad, J. Bedside Sonographic Measurement of Optic Nerve Sheath Diameter as a Predictor of Increased Intracranial Pressure in Children. eng. Annals of Emergency Medicine 53, 785-791 (2009).

46. Lee, S. U., Jeon, J. P., Lee, H. et al. Optic nerve sheath diameter threshold by ocular ultrasonography for detection of increased intracranial pressure in Korean adult patients with brain lesions. eng. Medicine 95, e5061 (2016).

47. Lochner, P., Falla, M., Brigo, F., Pohl, M. \& Strapazzon, G. Ultrasonography of the Optic Nerve Sheath Diameter for Diagnosis and Monitoring of Acute Mountain Sickness: A Systematic Review. eng. High altitude medicine $\mathcal{E}$ biology 16, 195-203 (2015).

48. Lochner, P., Fassbender, K., Lesmeister, M. et al. Ocular ultrasound for monitoring pseudotumor cerebri syndrome. eng. Journal of neurology 265, 356-361 (2018).

49. Lochner, P., Nardone, R., Tezzon, F., Coppo, L. \& Brigo, F. Optic nerve sonography to monitor treatment efficacy in idiopathic intracranial hypertension: a case report. eng. Journal of neuroimaging : official journal of the American Society of Neuroimaging 23, 533-534 (2013).

5o. Maissan, I. M., Ketelaars, R., Vlottes, B., Hoeks, S. E., den Hartog, D. \& Stolker, R. J. Increase in intracranial pressure by application of a rigid cervical collar: a pilot study in healthy volunteers. eng. European journal of emergency medicine : official journal of the European Society for Emergency Medicine 25, e24-e28 (2018)

51. Min, J. Y., Lee, J.-R., Oh, J.-T., Kim, M.-S., Jun, E.-K. \& An, J. Ultrasonographic assessment of optic nerve sheath diameter during pediatric laparoscopy. eng. Ultrasound in medicine $\mathcal{E}$ biology 41, 1241-1246 (2015).

52. Moretti, R. \& Pizzi, B. Optic Nerve Ultrasound for Detection of Intracranial Hypertension in Intracranial Hemorrhage Patients. eng. Journal of Neurosurgical Anesthesiology 21, 16-20 (2009).

53. Moretti, R., Pizzi, B., Cassini, F. \& Vivaldi, N. Reliability of optic nerve ultrasound for the evaluation of patients with spontaneous intracranial hemorrhage. eng. Neurocritical Care 11, 406-410 (2009).

54. Rajajee, V., Fletcher, J. J., Rochlen, L. R. \& Jacobs, T. L. Comparison of accuracy of optic nerve ultrasound for the detection of intracranial hypertension in the setting of acutely fluctuating vs stable intracranial pressure: post-hoc analysis of data from a prospective, blinded single center study. eng. Critical care (London, England) 16, R79 (2012).

55. Rajajee, V., Vanaman, M., Fletcher, J. J. \& Jacobs, T. L. Optic nerve ultrasound for the detection of raised intracranial pressure. eng. Neurocritical care 15, 506-515 (2011).

56. Rajajee, V., Williamson, C. A., Fontana, R. J., Courey, A. J. \& Patil, P. G. Noninvasive Intracranial Pressure Assessment in Acute Liver Failure. eng. Neurocritical care 29, 280-290 (2018).

57. Rehman Siddiqui, N. U., Haque, A., Abbas, Q., Jurair, H., Salam, B. \& Sayani, R. Ultrasonographic optic nerve sheath diameter Measurement for raised intracranial pressure in a Tertiary care centre of a developing country. eng. Journal of Ayub Medical College, Abbottabad: JAMC 30, 495-500 (2018).

58. Robba, C., Bacigaluppi, S., Cardim, D. et al. Intraoperative non invasive intracranial pressure monitoring during pneumoperitoneum: a case report and a review of the published cases and case report series. eng. Journal of Clinical Monitoring and Computing 30, 527-538 (2016).

59. Roque, P. J., Wu, T. S., Barth, L. et al. Optic nerve ultrasound for the detection of elevated intracranial pressure in the hypertensive patient. eng. The American Journal of Emergency Medicine 30, 1357-1363 (2012).

6o. Salahuddin, N., Mohamed, A., Alharbi, N. et al. The incidence of increased ICP in ICU patients with non-traumatic coma as diagnosed by ONSD and CT: a prospective cohort study. eng. BMC Anesthesiology 16, 106 (2016). 
61. Singleton, J., Dagan, A., Edlow, J. A. \& Hoffmann, B. Real-time optic nerve sheath diameter reduction measured with bedside ultrasound after therapeutic lumbar puncture in a patient with idiopathic intracranial hypertension. eng. American Journal of Emergency Medicine 33, 860.e5860.e7 (2015).

62. Školoudík, D., Herzig, R., Fadrná, T. et al. Distal enlargement of the optic nerve sheath in the hyperacute stage of intracerebral haemorrhage. eng. British Journal of Ophthalmology 95, 217-221 (2011).

63. Soldatos, T., Chatzimichail, K., Papathanasiou, M. \& Gouliamos, A. Optic nerve sonography: a new window for the non-invasive evaluation of intracranial pressure in brain injury. eng. Emergency medicine journal : EMJ 26, 630-634 (2009).

64. Steinborn, M., Friedmann, M., Makowski, C., Hahn, H., Hapfelmeier, A. \& Juenger, H. High resolution transbulbar sonography in children with suspicion of increased intracranial pressure. eng. Child's nervous system : ChNS : official journal of the International Society for Pediatric Neurosurgery 32, 655-66o (2016).

65. Tarzamni, M. K., Derakhshan, B., Meshkini, A. et al. The diagnostic performance of ultrasonographic optic nerve sheath diameter and color Doppler indices of the ophthalmic arteries in detecting elevated intracranial pressure. eng. Clinical Neurology and Neurosurgery 141, 82-88 (2016).

66. Tayal, V. S., Neulander, M., Norton, H. J., Foster, T., Saunders, T. \& Blaivas, M. Emergency department sonographic measurement of optic nerve sheath diameter to detect findings of increased intracranial pressure in adult head injury patients. eng. Annals of emergency medicine 49, 508-514 (2007).

67. Topcuoglu, M. A., Arsava, E. M., Bas, D. F. \& Kozak, H. H. Transorbital Ultrasonographic Measurement of Optic Nerve Sheath Diameter in Brain Death. eng. Journal of neuroimaging : official journal of the American Society of Neuroimaging 25, 906-9 (2015).

68. Blaivas, M., Theodoro, D. \& Sierzenski, P. R. Elevated intracranial pressure detected by bedside emergency ultrasonography of the optic nerve sheath. eng. Academic emergency medicine : official journal of the Society for Academic Emergency Medicine 10, 376-381 (2003).

69. Wang, L.-J. J., Chen, L.-M. M., Chen, Y. et al. Ultrasonography assessments of optic nerve sheath diameter as a noninvasive and dynamic method of detecting changes in intracranial pressure. eng. JAMA Ophthalmology 136, 250-256 (2018).

7o. Wang, L., Feng, L., Yao, Y. et al. Optimal optic nerve sheath diameter threshold for the identification of elevated opening pressure on lumbar puncture in a chinese population. eng. PLoS ONE 1o, 1-10 (2015).

71. Whiteley, J. R., Taylor, J., Henry, M., Epperson, T. I. \& Hand, W. R. Detection of elevated intracranial pressure in robot-assisted laparoscopic radical prostatectomy using ultrasonography of optic nerve sheath diameter. eng. Journal of neurosurgical anesthesiology 27, 155-159 (2015).

72. Yu, J., Park, J.-Y., Kim, D.-H. et al. Dexmedetomidine attenuates the increase of ultrasonographic optic nerve sheath diameter as a surrogate for intracranial pressure in patients undergoing robotassisted laparoscopic prostatectomy. eng. Medicine 98, e16772 (2019).

73. Hansen, H. C. \& Helmke, K. The subarachnoid space surrounding the optic nerves. An ultrasound study of the optic nerve sheath. Surgical and radiologic anatomy : SRA 18, 323-8 (1996).

74. Liu, D., Li, Z., Zhang, X. et al. Assessment of intracranial pressure with ultrasonographic retrobulbar optic nerve sheath diameter measurement. eng. BMC neurology 17, 188 (2017). 



\section{Development of an Automatic ONSD Algorithm}

Based on: Stevens, R.R.F., Gommer, E.D., Ariës, M., Ertl, M., Mess, W.H., Huberts, W. \& Delhaas, T. An Automated Algorithm for the Optic Nerve Sheath Diameter Assessment from B-mode Ultrasound Images (submitted). 


\section{Abstract}

The optic nerve sheath diameter (ONSD) is a promising surrogate marker for the detection of raised intracranial pressure (ICP). However, inconsistencies in manual ONSD assessment, such as ultrasound marker placement, are thought to affect ONSD and the corresponding ONSD cut-off values for the diagnosis of eleveated ICP, hereby hampering the full potential of ONSD in clinical practice. In this study, we developed an algorithm capable of estimating the ONSD automatically from Bmode ultrasound images at multiple depths. The algorithm uses intensity invariant asymmetry features to make an initial estimate of the borders of the sheath and subsequently applies an active contour to optimize the delineation of the sheath borders.

To evaluate the performance of the algorithm the outcomes were validated against manual ONSD measurements of two human experts. Each expert analyzed the images twice ( $\mathrm{M}_{1}$ and $\mathrm{M}_{2}$ ) in unknown order. The algorithm proved capable of segmenting the ONSD in 39/42 images, hereby showing mean differences of -0.08 $\pm 0.45 \mathrm{~mm}$ and $-0.05 \pm 0.41 \mathrm{~mm}$ compared to the averaged ONSD values $\left(\frac{M 1+M 2}{2}\right)$ of respectively, Operator 1 and Operator 2, whereas the mean difference between the two experts was $0.03 \pm 0.26 \mathrm{~mm}$.

Moreover, differences between algorithm-derived and expert-derived ONSD values were found to be much smaller than the $1 \mathrm{~mm}$ difference that is expected between patients with normal and elevated ICP $[1-3]$. It is therefore likely that our algorithm can distinguish between patients with normal and elevated ICP. The presented algorithm eliminates both the inter- and intraobserver variability associated with manual ONSD assessment because the algorithm has no intrinsic assessment variability. Therefore, our automated ONSD assessment has the potential to improve the accuracy of ONSD as a surrogate marker for elevated ICP. However, future research should be carried out to validate if the algorithm-derived ONSD values do indeed correlate better with ICP and result in more accurate non-invasive ICP predictions. 


\subsection{Introduction}

H E optic nerve sheath diameter (ONSD) is a promising surrogate marker for non-invasive estimation of intracranial pressure (ICP) [4-7] and is therefore often measured in clinical practice. The ONSD is manually assessed from ultrasound B-mode images, mostly at a depth of $3 \mathrm{~mm}$ from the papilla because that region is assumed to be most sensitive to changes in ICP[8]. Although the intraand interobserver variability in ONSD estimation can be low within individual studies[9, 10], ONSD cut-off values for the diagnosis of elevated ICP (>15 $\mathrm{mmHg}$ ) vary between studies[11-13]. A possible explanation could be the discrepancies in ONSD assessment methodologies between different centers[13, 14] and the lack of agreement on the echoic behavior of the different layers of the optic nerve sheath (see Chapter 2). Although ONSD assessment might be improved by standardization according to respective guidelines, the ONSD values would still be dependent on the compliance of the operator. Differences in manual ONSD measurements might be overcome by constructing an automated framework for ONSD assessment.

Automated approaches on ONSD segmentation from ultrasound images have already been reported in literature[15-17]. In contrast to Gerber et al. [15] and Soroushmehr et al. [16], Meiburger et al. [17] developed an algorithm that was able to segment the outlines of both the optic nerve and the optic nerve sheath instead of determining only a single ONSD value at a depth of $3 \mathrm{~mm}$. Derivation of the ONSD at a wide range of depths allows investigation of the sensitivity of ONSD to changes in ICP at multiple depths, which might lead to new insights. The intraobserver variability of the algorithm was zero while the interobserver variability between the algorithm and manual expert measurements was comparable to the interobserver variability between experts reported in literature.

Although the approach by Meiburger et al. [17] is promising, we observed that our ocular ultrasound images displayed a less clear distinction between the hyperechoic bands and the retrobulbar fat in 37/42 images. Hence, detecting the outline of the hyperechoic bands using intensity thresholds as proposed by Meiburger et al. [17] would be difficult in our images.

In this study we therefore developed and evaluated an automatic ONSD algorithm that is hypothesized to be less sensitive to such differences in intensities.

\subsection{Materials and Methods}

\subsubsection{Image acquisition and patient data}

A total of 42 B-mode transocular ultrasound images were acquired from 26 comatose patients suffering from traumatic brain injury or subarachnoid hemorrhage 
who were admitted to the intensive care unit of the Maastricht University Medical Center (Maastricht, the Netherlands). The study was approved by the local ethical committee (METC 16-04-243) and written informed consent was obtained for all participants. Images were acquired with a Philips iU22 ultrasound device (Eindhoven, The Netherlands) and a 17-5 MHz linear transducer by two investigators who had practised on at least 20 subjects. Measurements were performed on both eyes (if possible) by placing the transducer horizontally on the closed eyelid with the patient in supine position and the head tilted at an angle of $20-30^{\circ}$. The transducer angle was adjusted to find the best possible image of the optic nerve, i.e. the optic nerve in a vertical orientation with the hyperechoic bands visible on both sides of the nerve. To prevent tissue damage the mechanical index (MI) was always kept lower than o.3. Finally, the images were exported from the ultrasound machine to a computer for offline assessment.

\subsubsection{Automated algorithm}

The algorithm was developed in Matlab(R2018a, The MathWorks, Inc., Natick, Massachusetts, United States) and consists of six consecutive steps:

- Step 1: Automatic cropping

- Step 2: Image enhancement

- Step 3: Feature extraction

- Step 4: Initial fat and sheath detection

- Step 5: Segmentation of the outline using an active contour

- Step 6: Optic nerve sheath diameter calculation

\section{Step 1: Automatic cropping}

To facilitate automatic seedpoint selection later on, the region containing the optic nerve $(\mathrm{ON})$ and its sheath was localized and extracted from the original B-mode image. Hereto, the image was made black and white using a threshold of 0.26 [-] (Figure 3.1A-B). Next, the distal part of the globe and the corresponding retrobulbar structures were extracted by searching for the boundaries of the largest object within this image. This was done by locating the first and last white pixel for each row and column within the image (Figure 3.1B). Next, an estimation of the ON location was made by searching for the vertical B-mode intensity line with the most black pixels. Thereafter, the nerve midpoint, i.e. the intersect between this intensity line and the retina, was determined. Subsequently, the image was cropped, 


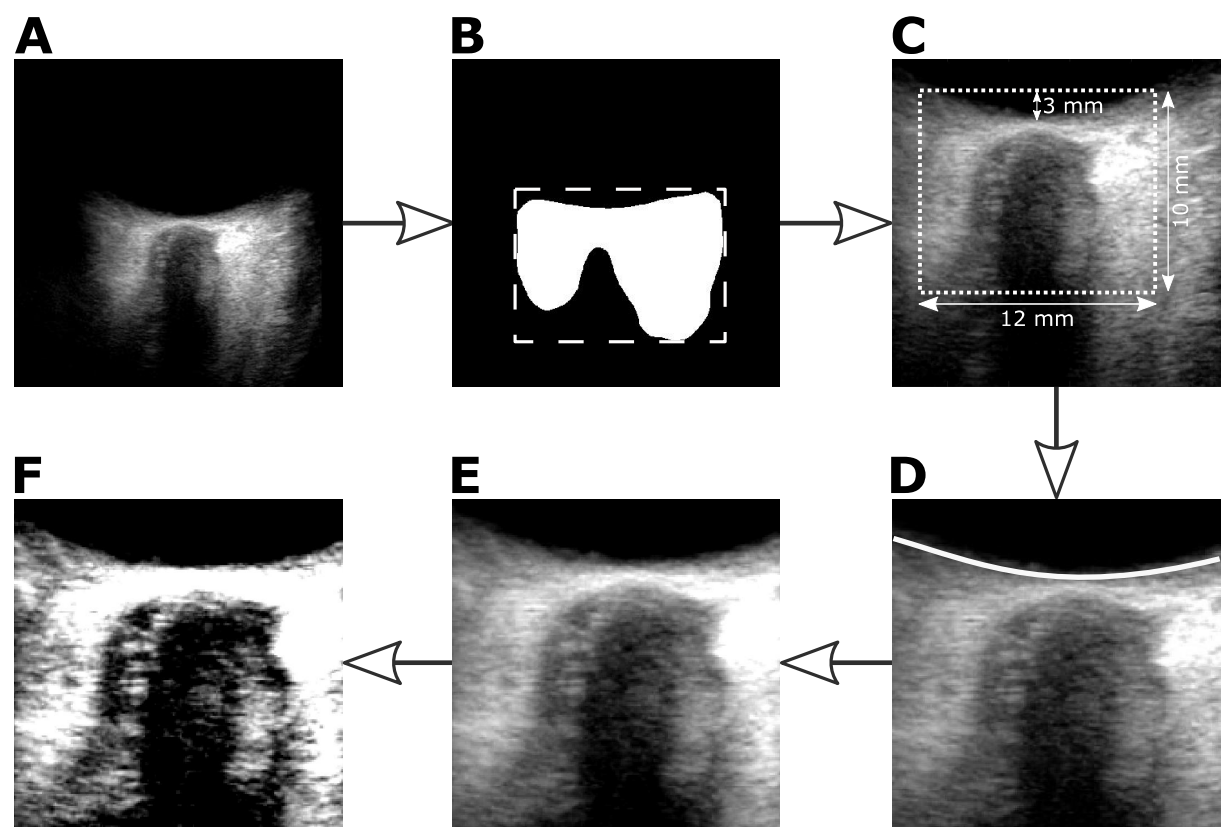

Figure 3.1: The several steps taken to automatically select and enhance the region that contains the optic nerve and its sheath from the B-mode ultrasound image: A) Original B-mode ultrasound image, B) Detection of the outline of the retrobulbar structures used for the initial image crop, C) Detection of the optic nerve midpoint, i.e. crossing of the nerve midline and the retina (white arrow). Next the image is cropped using a width of $12 \mathrm{~mm}$ and a depth of $10 \mathrm{~mm}$ around the nerve midpoint, D) Full retina detection and fitting of a second order polynomial (white line), E) Bandpass filtering of the cropped image, F) image enhancement of the bandpass filtered image.

first in the $x$-direction using an arbitrary chosen width of $12 \mathrm{~mm}$ around the ON location, and then in the $y$-direction using a height corresponding to a depth of $10 \mathrm{~mm}$ from the retina (Figure 3.1C). Next, the pixels corresponding to the retina were detected by a search for the first white pixel within each of the vertical B-mode intensity lines followed by the fitting of a second degree polynomial curve through these points (Figure 3.1D).

\section{Step 2: Image enhancement}

In high quality images the hyperechoic bands are separated from the hyperechoic retrobulbar fat by a thin dark line. In practice this dark line is not always clearly visible causing the hyperechoic bands and retrobulbar fat to blend into each other which hampers accurate detection of the outer border of the hyperechoic striped 
bands. Therefore, we first enhanced the image to increase the contrast between these two structures. Hereto, we applied a 2D Butterworth bandpass filter with cut-off frequencies of 5 and $10 \mathrm{~Hz}$, respectively (Figure 3.1E). Subsequently, the image was sharpened by the subtraction of a Gaussian blurred image ( $\mu=0$ and $\sigma=20$ pixels) from the bandpass filtered image (Figure $3.1 \mathrm{~F}$ ).

\section{Step 3: Feature extraction}

Despite image enhancement, segmentation of structures in ultrasound images can still be difficult due to, for example, differences in intensities or the presence of speckle or artefacts[18]. To overcome this intensity dependency we chose to detect the borders of the structures of interest by using asymmetry features calculated from the monogenic signal of the image (see Appendix 3.A). The monogenic signal is a transformation of the original image into three new images that can be used to calculate the local phase and amplitude and which, on their turn, can be used to derive several useful features, e.g. asymmetry or local phase congruency. Especially, the asymmetry features are useful for edge detection. To specify the scale (wavelengths $\lambda$ in pixels) of the detected asymmetry features, we incorporated a bandpass filter in the calculation of the monogenic signal. This can be very useful to distinguish between clinically relevant image structures and image structures caused by artefacts[18, 19]. As bandpass filter we chose the (even) log-Gabor filter[18-20]:

$$
G_{e}(\boldsymbol{\omega})=\exp \left(-\frac{\left(\log \left(\frac{|\boldsymbol{\omega}|}{\omega_{0}}\right)\right)^{2}}{2\left(\log \left(\sigma_{0}\right)\right)^{2}}\right),
$$

where $|\cdot|$ denotes the $L^{2}$-norm and $\boldsymbol{\omega}=\left(\omega_{x}, \omega_{y}\right)^{T}$ are the frequencies in the $2 \mathrm{D}$ frequency domain of the image in respectively, the image directions $x$ and $y$. Moreover, $\omega_{0}$ is the center frequency of the bandpass filter and $\sigma_{0}$ is the shape parameter that controls the width of the filter. The derived monogenic signal $\mathbf{f}_{m}(\mathbf{x})$ can be written as a combination of one even image and two odd images:

$$
\mathbf{f}_{m}(\mathbf{x})=\left[\begin{array}{c}
f_{e}(\mathbf{x}) \\
f_{o_{x}}(\mathbf{x}) \\
f_{o_{y}}(\mathbf{x})
\end{array}\right],
$$

with $\mathbf{x}=(x, y)^{T}$ the pixel coordinates of the image. The two different odd parts $\left(f_{o_{x}}(\mathbf{x})\right.$ and $\left.f_{o_{y}}(\mathbf{x})\right)$ show changes within the image that have wavelengths close to $\lambda_{0}=\frac{2 \pi}{\omega_{0}}$ in respectively, the $x$ - and $y$-direction of the image.

The direction of asymmetry around an edge, i.e. whether the image feature changes 
from low to high or from high to low, allows us to distinguish between the borders of the fat and the borders of the hyperechoic bands (Figure 3.2). Therefore, we used the Kovesi's signed feature asymmetry which takes into account the direction of asymmetry. Kovesi's signed feature asymmetry $\hat{R}(\mathbf{x})$, has values between -1 and +1 . Values close to -1 or 1 correspond to "changes" that are respectively, troughs or peaks. The asymmetry feature can be calculated as follows[20]:

$$
\hat{R}_{j}(\mathbf{x})=\sum_{i} \frac{\left\lfloor\left|f_{o, \lambda_{i}}(\mathbf{x})\right|-\left|f_{e, \lambda_{i}}(\mathbf{x})\right|-T\right\rfloor}{A_{\lambda_{i}}(\mathbf{x})+\epsilon} \frac{f_{o_{j}, \lambda_{i}}(\mathbf{x})}{\left|f_{o, \lambda_{i}}(\mathbf{x})\right|} .
$$

Here, $j$ denotes either the first or second odd image in respectively, the $x$ - or $y$ direction, $\lambda_{i}$ is the set of wavelengths used to calculate the monogenic signal, and $T$ is a threshold that determines the sensitivity which was set to 0.18 [20]. In addition, $\lfloor\cdot\rfloor$ is a shorthand indicating that values smaller than zero are set to zero, and $\epsilon$ is a positive small number that prevents division by zero. Moreover, the term $f_{o}(\mathbf{x})$ is the combined odd component given by:

$$
f_{o, \lambda_{i}}(\mathbf{x})=\sqrt{f_{o_{x}, \lambda_{i}}(\mathbf{x})^{2}+f_{o_{y}, \lambda_{i}}(\mathbf{x})^{2}}
$$

and, $A_{\lambda_{i}}(\mathbf{x})$ is the amplitude of the combined odd component and the even component:

$$
A_{\lambda_{i}}(\mathbf{x})=\sqrt{f_{e, \lambda_{i}}(\mathbf{x})^{2}+f_{o, \lambda_{i}}(\mathbf{x})^{2}} .
$$

\section{Step 4: Initial fat and sheath detection}

The next step was the detection of the external outlines of the hyperechoic striped bands and the internal outline of the hyperechoic retrobulbar fat. First of all, the signed asymmetry features in both the $x$ - and $y$-direction were derived from the monogenic signal calculated from the enhanced B-mode images using $\lambda_{i}=$ $12^{i}, i=0,1, \ldots, 6 \mathrm{~mm}$ and $\sigma_{0}=0.35$. An example of the $x$-directed signed asymmetry features for one of our images can be seen in Figure 3.2A.

As can be seen in Figure 3.2A, the left border of the hyperechoic band is a peak ( white $=$ positive) whereas the left border of the fat is a trough (black = negative). Secondly, initial points corresponding to the outline of the retrobulbar fat and sheath were located. Hereto, we performed a row-wise search starting from the middle of the image, i.e. the vertical midline of the optic nerve, towards the left side of the image and annotated the first peak after a trough as sheath and the second trough in each row as fat (Figure 3.2B and C). Subsequently, we removed possible outliers by detecting and removing points that deviated more than three standard deviations from the median. The right side edges of the hyperechoic band and fat were detected in the same way but taking into account the change of sign. 


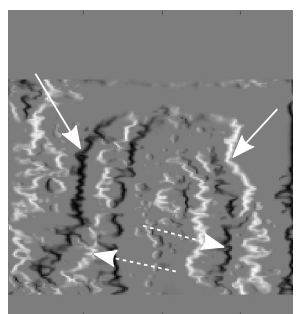

A

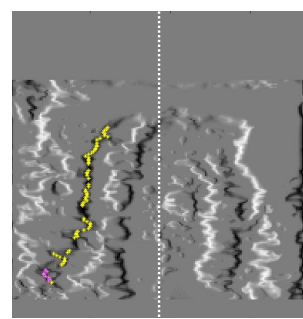

B

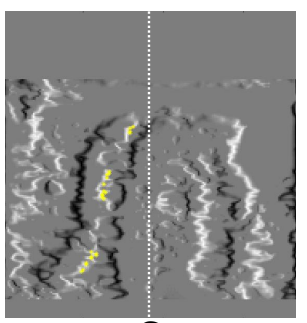

C

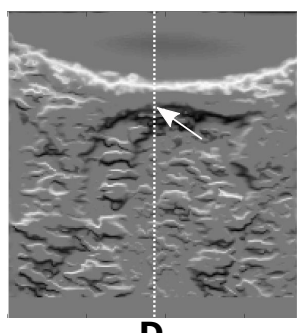

D

Figure 3.2: Panel A: Signed asymmetry features of the enhanced cropped image. The borders corresponding to the retrobulbar fat are denoted with white solid arrows. The borders corresponding to the hyperechoic bands are indicated by the dotted arrows. Note the differences in sign (white = positive and dark = negative) of the left and right edges. Panel B and C : Detection of the edges corresponding to the border of respectively, the retrobulbar fat and the hyperechoic bands. Yellow points denote the found edges, whereas pink points are points that are discarded because they are deemed to be outliers. Panel D: Detection of the edge corresponding to the "dome" of the nerve (white arrow). The white dotted line denotes the vertical midline of the optic nerve.

To obtain a better estimation of the sheath outline at the top of the nerve we also wanted to segment the "dome of the nerve" in the next stage. Hereto, we determined a point located at the edge of the dome by finding the crossing of the nerve midline with the first trough found from the retina down on the $y$-directed signed asymmetry image (see Figure 3.2D).

\section{Step 5: Segmentation}

To obtain a better estimate of the edges and to allow detection of the borders on locations where no initial outline was found, we evolved a contour $C$ along the edges of interest. We used the localized active contour as described by Lankton et al. [21] and implemented in MATLAB by Pang [22], hereby using the annotated points from the previous step as initialization for the contour. Optimization of the active contour was done by minimizing the "energy" of the active contour. In its general form the "energy" of the active contour is given by:

$$
E_{\text {contour }}(\mathbf{C})=\int_{\Omega_{x}} F_{\text {image }}(\mathbf{x}) d x+\int_{0}^{1} \alpha F_{\text {penalty }}(\mathbf{x}(\vec{s})) d s .
$$

Here $C$ is a closed contour defined as the zero-level set of the signed distance function $\phi$, i.e. $C=\{\mathbf{x} \mid \phi(\mathbf{x})=0\}$ with $\mathbf{x}=(\vec{x}, \vec{y})^{T}$ the set of all pixel coordinates of the image $I$ given by the domain $\Omega_{x}$, and $\vec{s}$ are the parametrized points on the contour. Moreover, $F_{\text {image }}(\mathbf{x})$ is the "force" of the image which "pulls" the contour towards the edges, and $F_{\text {penalty }}(\mathbf{x}(\vec{s}))$ is a penalty function that governs the 
smoothness of the contour with its scaling factor $\alpha$. As image force the Chan-Vese energy [23] is used, which is given by:

$$
F_{\text {image }}(\mathbf{x})=\int_{\Omega_{i}}\left(I(\mathbf{x})-\mu_{\text {in }}\right)^{2} d x+\int_{\Omega_{o}}\left(I(\mathbf{x})-\mu_{\text {out }}\right)^{2} d x .
$$

Here $\Omega_{i}$ and $\Omega_{o}$ are all the pixels that lie respectively, in- and outside of the contour, i.e. the local interior and exterior. The parameters $\mu_{i n}$ and $\mu_{\text {out }}$ are the expected (mean) values of the pixels on the domains $\Omega_{i}$ and $\Omega_{o}$ (see Figure 3.3). Following Lankton et al. [21], the arc length was used as a penalty function. Finally, the image force was localized using a mask $B(\vec{x}, \mathbf{y})$ so the evolution of the contour is only influenced by local information:

$$
E_{\text {contour }}(C)=\int_{\Omega_{x}} \int_{\Omega_{y}} B(\vec{x}, \mathbf{y}) F_{\text {image }}(\mathbf{y}) d y d x+\alpha \int_{0}^{1}\|\nabla \mathbf{x}(\vec{s})\| d s,
$$

where $B(\vec{x}, \mathbf{y})$ is a circle with radius $r$ around point $\vec{x}$, and $\mathbf{y}$ is a second set of pixel coordinates that lie within the domain $\Omega_{y}$ of the mask.

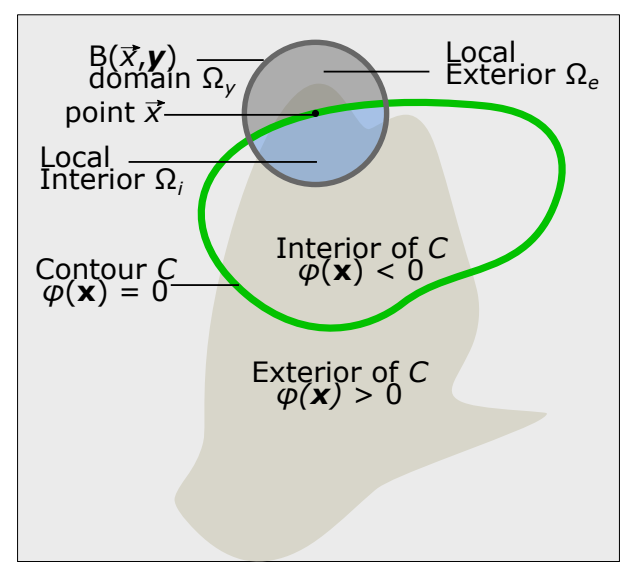

Figure 3.3: Schematic drawing of the different components used to derive the forces that determine the movement of the contour. Image adopted from[21]. 

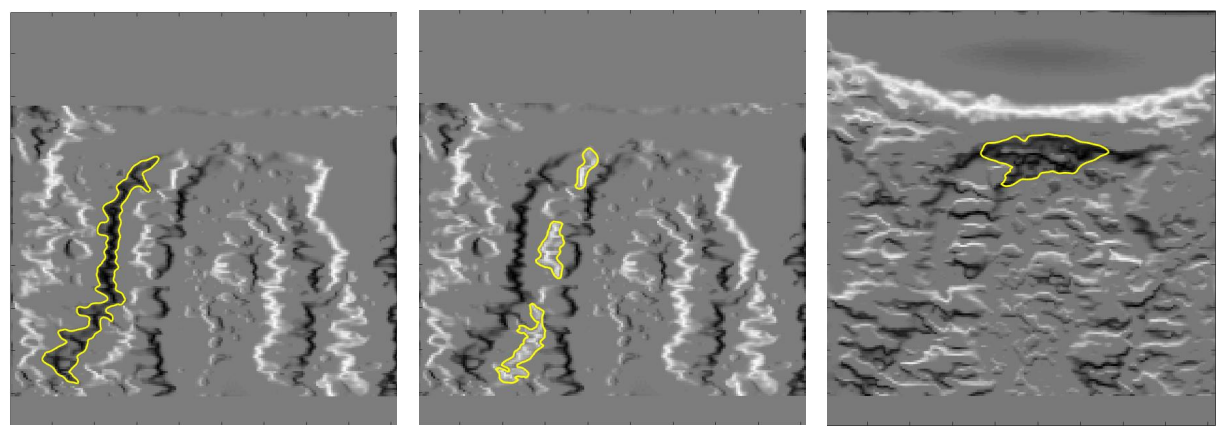

Figure 3.4: An example showing the snake evolution segmenting the border corresponding to the retrobulbar fat (left), the sheath (middle), and the "dome" of the nerve (right).

Using the energy function described above, the sheath, fat, and dome were segmented using the following parameters: the penalty constant $\alpha$ was set to 0.05 for the sheath and fat, and to 0.3 for the dome segmentation. The radius of the mask was set to 20 pixels for all three segmentations. To control the computational time of the gradient-descent method used to minimize the energy of the contour the maximum number of iterations were set to 100,400 , and 150 for respectively, sheath, fat, and dome. An example of the segmentations is shown in Figure 3.4.

\section{Step 6: ONSD calculation}

After the five contours, i.e. left and right fat, left and right sheath, and the dome, were evolved, an initial outline of the optic nerve and the sheath was determined. Hereto, the inner edges of the left and right fat segmentation and the outer edges of the left and right sheath segmentation were localized at multiple depths perpendicular to the nerve midline. When no sheath outline was found at a given depth, the fat outline at that depth was used instead. In addition, the superior edge of the dome contour was detected by a search in the vertical direction. Finally, all outline points were combined into one curve and smoothed with a smoothing spline (smoothn function in Matlab with a high smoothing parameter). Finally, the ONSD was calculated at multiple depths by taking the distance between the left and right outline (see Figure 3.5). 

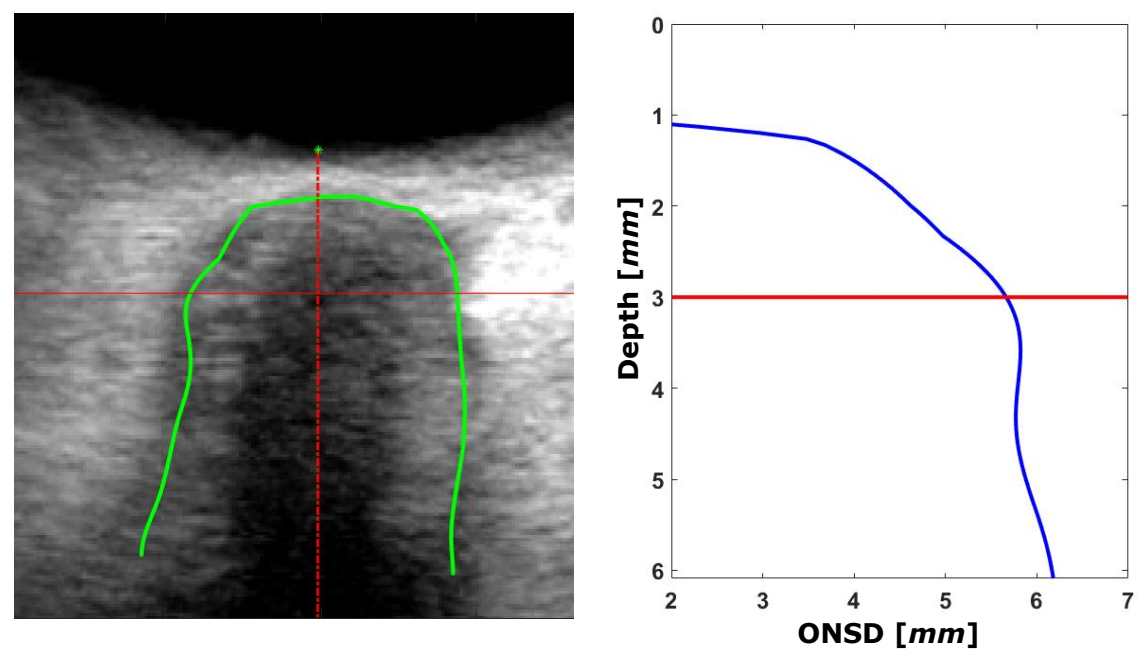

Figure 3.5: Final delineation of the optic nerve and its sheath (left). The corresponding ONSD as function of the depth (right).

\subsubsection{Assessment of algorithm performance}

To assess the quality of the automated ONSD algorithm, we compared the automatic algorithm-derived ONSD values with ONSD values from manual assessments performed by two human experts (Operator 1 and Operator 2). Manual assessments by the experts were performed in Matlab using an in-house developed framework. This framework was built such that it resembled ONSD segmentation on a real ultrasound scanner as closely as possible. First, an ultrasound marker had to be placed at the retina in line with the optic nerve. Subsequently, a second marker appeared which needed to be dragged down in order to determine the depth of $3 \mathrm{~mm}$. Next, two new markers appeared which had to be dragged towards the anatomical landmarks that were assumed to correspond with the outline of the optic nerve sheath. Upon confirmation, the Euclidean distance between the two markers was saved as the corresponding ONSD value.

Both experts analyzed the 42 ultrasound images twice in unknown order while blinded to any patient information.

First of all, we assessed the intraobserver variability, i.e. the agreement between ONSD values obtained when the same image is analyzed twice by the same rater, for each expert and for the automated algorithm. Hereto, we calculated the coefficient of determination $\left(R^{2}\right)$ for the linear regression model $y=x$ where $x$ and $y$ represent respectively, the first and second measurement (M1 and M2). In addition, we 
determined the mean difference and limits of agreement using Bland-Altman plots. Logically, the intraobserver variability of the algorithm is zero as the algorithm yields the same ONSD value every time the same image is analyzed.

Secondly, we examined the interobserver variability, i.e. the agreement between ONSD values obtained when the same image is analyzed by two different raters, between the experts and the algorithm, and between the experts themselves. However, two different analyses were carried out. In the first analysis we calculated the mean of the two repeated manual ONSD assessments for each expert (mOpi and mOp2) and compared these values between the two experts ( $\mathrm{mOp} 1-\mathrm{mOp} 2)$ and with the ONSD values derived by the algorithm (mOp1 - A and mOp2 - A). In the second analysis we only considered the first measurement of the operators (Op1 and Op2) to evaluate the effect of repeated measurements and to enable comparison with reported results in literature that were based on single measurements. Similarly as for the intraobserver variability, linear regression models $y=x$ were made for each analysis, i.e. for the comparisons between Operator 1 and the algorithm, Operator 2 and the algorithm, and Operator 1 and Operator 2 . Subsequently, the corresponding coefficients of determination were calculated. Moreover, the corresponding mean difference and limits of agreement were derived from Bland-Altman plots. Finally, we investigated if the mean ONSD values of the Algorithm, Operator 1 , and Operator 2 were statistically different, hereby using the Wilcoxon signed rank test because the data was not normally distributed.

\subsection{Results}

Accurate segmentation of the B-mode ultrasound images was possible in 39 out of the 42 images, resulting in a success-rate of almost $93 \%$. In two images the segmentation clearly failed because of a dark image artefact just below the retina that masked the true outline of the hyperechoic band and retrobulbar fat on one side, whereas in another image the physical orientation of the optic nerve deviated largely from the vertical midline assumed in our algorithm resulting in a very large ONSD value. These three images were excluded from further analysis.

The means and standard deviations of the ONSD calculated over all the images are given in Table 3.1. Although the mean ONSD value of the algorithm is slightly higher than the ONSD values derived by the experts, these differences were found to be non-significant.

The Bland-Altman plots used to analyze the interobserver variabilities between the averaged repeated ONSD measurements of Operator 1 (mOp1), Operator 2 (mOp2), and the ONSD values obtained with the algorithm (A) are given in Figure 3.7 and in Table 3.2. The ONSD values determined by the automatic algorithm were on average slightly higher than the manually-derived values, i.e. mean differences of 
Table 3.1: The mean and standard deviation of the ONSD values [mm] calculated over all the images.

\begin{tabular}{llll}
\hline ONSD & Algorithm & Operator 1 & Operator 2 \\
\hline First Measurements & $5.42 \pm 0.54$ & $5.30 \pm 0.4$ & $5.40 \pm 0.46$ \\
Second Measurements & & $5.38 \pm 0.54$ & $5.34 \pm 0.5$ \\
Averaged Measurements & & $5.34 \pm 0.47$ & $5.37 \pm 0.45$ \\
\hline
\end{tabular}

No significant differences ( $\mathrm{p}>0.05$ ) were found between the algorithm and the experts using the Wilcoxon signed rank test.

$-0.08 \pm 0.45 \mathrm{~mm}$ and $-0.05 \pm 0.41 \mathrm{~mm}$ when compared with the averaged manual expert assessments of respectively, Operator 1 and Operator 2. In comparison, Operator 1 showed a mean difference of $-0.03 \pm 0.26 \mathrm{~mm}$ with respect to Operator 2. In addition, the corresponding $R^{2}$-values are $0.37,0.44$, and 0.71 for respectively, Operator 1 vs Algorithm, Operator 2 vs Algorithm, and Operator 1 vs Operator 2. The interobserver variabilities of the comparison between the first measurement of Operator $1(\mathrm{Op} 1)$, Operator $2(\mathrm{Op} 2)$, and the algorithm $(\mathrm{A})$ are also given in Table 3.2. Comparing only the first manual measurements slightly increased the interobserver variabilities between the observers and the algorithm to -0.12 \pm 0.47 $\mathrm{mm}$ and $-0.02 \pm 0.41 \mathrm{~mm}$ for respectively, Operator 1 and Operator 2, whereas the difference between the experts increased to $-0.1 \pm 0.30 \mathrm{~mm}$.

The intraobserver variabilities of Operator 1 and Operator 2 are shown in Figure 3.6. The first measurements of Operator 1 showed a mean difference of -0.08 \pm 0.33 $\mathrm{mm}$ compared to the second measurements. In addition, the first measurements of Operator 2 showed a mean difference of $0.06 \pm 0.30 \mathrm{~mm}$ compared to the second measurements. The corresponding $R^{2}$-values were 0.63 for Operator 1, and 0.65 for Operator 2. Obviously, the algorithm has no intraobserver variability because the algorithm yields exactly the same ONSD value when the same image is analyzed twice.

\subsection{Discussion}

\subsubsection{Major Findings}

In this study we developed an algorithm that allows for automatic optic nerve sheath diameter (ONSD) assessment from B-mode ultrasound images. Using asymmetry features we were able to segment the ONSD at a range of depths in $93 \%$ of the images in which the sheath outline was often poorly visible.

To validate the ONSD values obtained with the algorithm, these values were compared to manual ONSD assessements performed by two experts. The intraobserver variability of both our operators $(0.33 \mathrm{~mm}$ and $0.30 \mathrm{~mm})$ was only a bit larger than 
the ones reported by Bäuerle et al. [9] (0.22 mm) and the $R^{2}$ of both operators was comparable to the values reported by Bäuerle et al. [9, 24], indicating representative expert assessments.

Mean ONSD derived by the algorithm was slightly higher than the mean ONSD values derived by the experts, however, these differences were not statistically significant, indicating that the algorithm-derived ONSD values were physiological.

The interobserver variabilities obtained in our study and the ones found in literature are given in Table 3.2. Overall, the interobserver variabilities between the two experts found in this study are comparable with values reported in literature. The interobserver variabilities between the experts and the algorithm are a bit larger than our interobserver variabilities between the two experts, however, similarly as Meiburger et al. [17], the difference between expert-derived ONSD values and algorithm-derived ONSD values is much smaller than the $1 \mathrm{~mm}$ differences in ONSD values between patients with normal and elevated ICP reported in literature[1-3]. This makes it likely that our algorithm can stratify between patients with elevated ICP and patients with normal ICP. Therefore, our algorithm can play an important role in improving the ONSD cut-off values for the detection of elevated ICP.

Compared to the algorithm presented by Meiburger et al. [17] we found a slightly smaller interobserver variability between the ONSD values derived with the algorithm and the expert ONSD values, whereas the $R^{2} \mathrm{~s}$ of the linear regression between our algorithm and experts were comparable to the values reported by

Operator 1

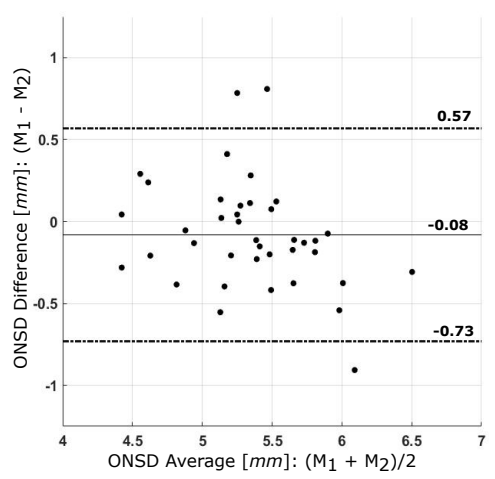

Operator 2

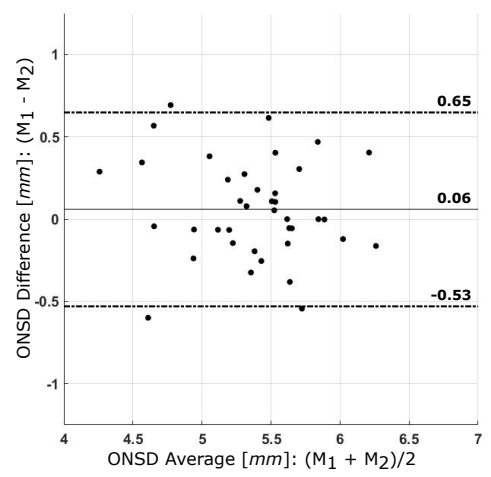

Figure 3.6: Bland-Altman plots comparing the two measurements of the optic nerve sheath diameter (M1 and $\mathrm{M}_{2}$ ) for Operator 1 (left) and Operator 2 (right). Solid lines depict the mean of the differences, dashed lines denote the limit of agreement. 
Meiburger et al. [17]. Similarly as Meiburger et al. [17] our approach allows segmentation of the outline of the optic nerve sheath when the hyperechoic bands are non-continuous, i.e. not visible on every depth. In contrast to Meiburger et al. [17], our intensity invariant features enable us to also segment the outline of the optic nerve sheath when the thin dark line separating the retrobulbar fat and the hyperechoic bands is poorly visible, i.e. the retrobulbar fat and hyperechoic bands blend into each other.
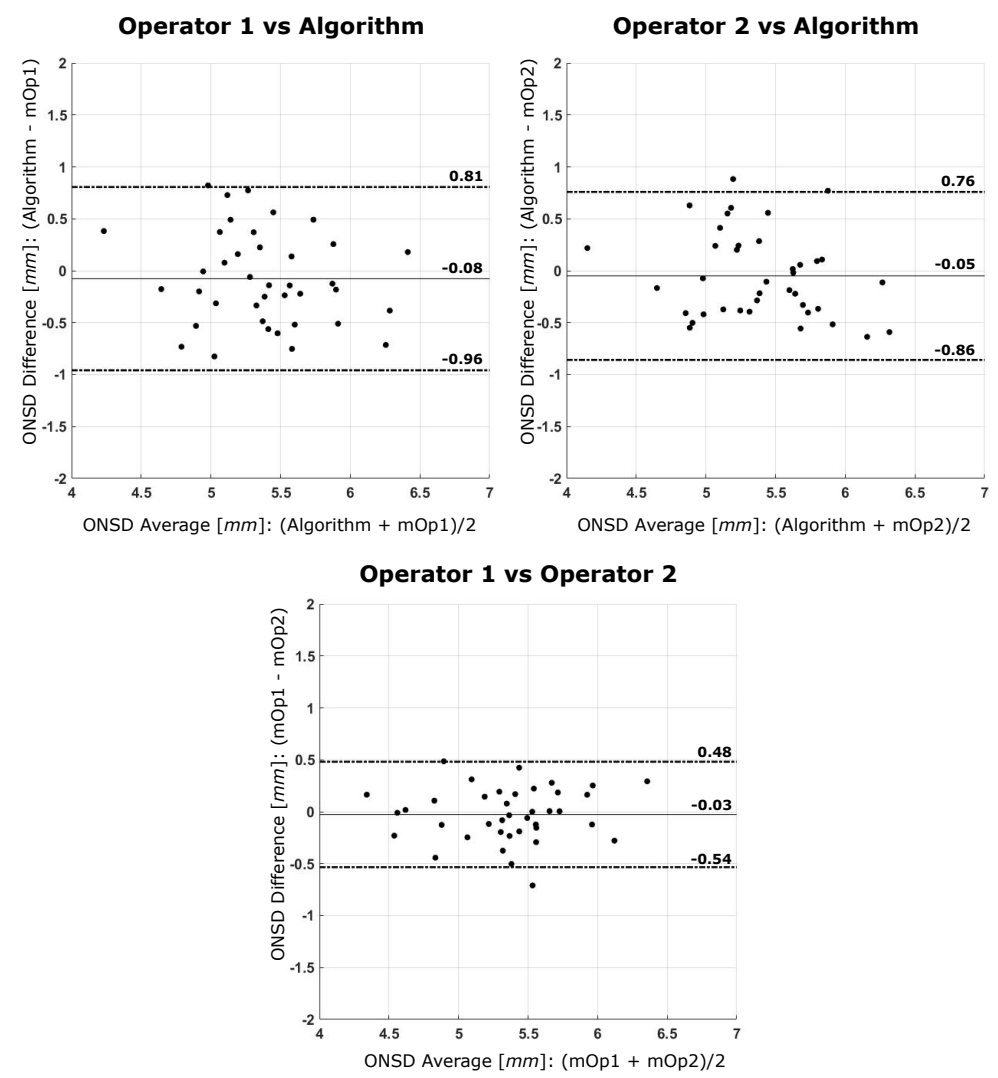

Figure 3.7: Top row: Bland-Altman plots comparing the mean of the two manual assessments by Operator 1 with the automatic measurement (top left), the mean of the two manual assessments by Operator 2 with the automatic measurement (top right), and the mean of the two manual assessments by Operator 1 with the mean of the two manual assessments by Operator 2 (bottom). Solid lines depict the mean of the differences, dashed lines denote the limits of agreement. 
Table 3.2: Overview of the interobserver variability found in literature and the values obtained in the current study.

\begin{tabular}{lllllll}
\hline & & \multicolumn{5}{c}{ Interobserver variability } \\
\cline { 2 - 7 } Author & Comparison & Bias & SD & \multicolumn{2}{c}{ LOA } & $R^{2}$ \\
\hline Lochner et al. [10] & Op1 - Op2 (L) & 0.08 & 0.28 & 0.48 & -0.64 & - \\
& Op1 - Op2 (R) & 0.09 & 0.40 & 0.71 & -0.90 & - \\
Bäuerle et al. [24] & Op1 - Op2 & 0.25 & 0.51 & 1.25 & -0.75 & 0.67 \\
Bäuerle et al. [9] & Op1 - Op2 (L) & 0.10 & 0.38 & 0.84 & -0.64 & 0.66 \\
& Op1 - Op2 (R) & 0.04 & 0.37 & 0.77 & -0.69 & 0.71 \\
Meiburger et al. [17] & Op1 - Op2 & $0.44^{*}$ & 0.52 & 1.46 & -0.58 & 0.42 \\
& Op1 - A & 0.06 & 0.52 & 1.07 & -0.95 & 0.41 \\
& Op2 - A & -0.38 & 0.55 & 0.69 & -1.44 & 0.37 \\
Our work & mOp1 - mOp2 & -0.03 & 0.26 & 0.48 & -0.54 & 0.71 \\
& mOp1 - A & -0.08 & 0.45 & 0.81 & -0.96 & 0.37 \\
& mOp2 - A & -0.05 & 0.41 & 0.76 & -0.86 & 0.44 \\
& Op1 - Op2 & -0.10 & 0.30 & 0.48 & -0.67 & 0.63 \\
& Op1 - A & -0.12 & 0.47 & 0.81 & -1.04 & 0.31 \\
& Op2 - A & -0.02 & 0.41 & 0.78 & -0.82 & 0.46 \\
\hline
\end{tabular}

$\mathrm{L}$ and $\mathrm{R}$ denote a comparison for the ONSD values of respectively, the L and R eye only, and LOA are the limits of agreement. In addition, mOp denotes that the repeated ONSD measurements performed on the images by the Operator are averaged whereas Op denotes that only the first manual ONSD measurement of the operator is considered. Furthermore, the letter A stands for Algorithm.

* The large bias is most likely caused because Op1 was experienced whereas Op2 was less experienced.

The comparison between single and repeated ONSD measurements shows that ONSD measurements performed by two observers deviate more from each other when these ONSD values are obtained using non-repeated measurements. In line with this observation, most studies calculate the mean of multiple ONSD measurements which increases the time it takes to perform manual ONSD measurements[17]. Similarly as the algorithm of Meiburger et al. [17], our algorithm has no intrinsic variability and therefore one singular measurement is sufficient to obtain an accurate ONSD value. This does not only reduce the time it takes to perform ONSD assessments but also overcomes the variation within the ONSD values due to intra- and interobserver variabilites associated with manual ONSD assessment. 


\subsubsection{Limitations and Future work}

Our study has a few limitations. Most important is the fact that we did not investigate if our algorithm indeed leads to a more accurate detection of elevated ICP. Although our algorithm eliminates the intra- and interobserver variabilities present within the manually-derived ONSD values, automated ONSD assessment might not be enough to obtain accurate ONSD cut-off values for the detection of elevated ICP because the inconsistencies in manual delineation of the optic nerve sheath only represent part of the methodological differences reported in literature[13, 14, 25, 26]. Also differences in image acquisition, such as the ultrasound machine, the ultrasound transducer, and the experience of the operator can have an impact on the ONSD values. In order to obtain more accurate ONSD values it is therefore necessary to not only utilize automatic segmentation algorithms, but also to standardize the transocular ultrasound acquisition[14, 17].

It would also be of interest to evaluate our automated algorithm in multiple clinical centres because images from different centres might contain image characteristics for which the algorithm is not developed and this could hamper the algorithm's performance.

Moreover, for now we assumed the nerve midline to be vertically oriented. Though the optic nerve was orientated approximately vertical in most of our ultrasound images, it would be more accurate to calculate the ONSD using a correctly angled optic nerve midline. This would also allow us to obtain correct ONSD values from images with a very tilted optic nerve, such as the image we now excluded. This will probably further improve the agreement with the expert ONSD values.

In contrast to Meiburger et al. [17] we did not segment the diameter of the optic nerve (OND) itself. Although the OND can be useful for the diagnosis of neurological disorders, we focused on the segmentation of the ONSD. Nevertheless, assessment of the OND can easily be incorporated in our algorithm by finding the first negative peak from the midline in our asymmetry images.

Another limitation is that the automatic segmentation failed in two images because a dark image artifact below the retina obscured the outline of the hyperechoic band and the retrobulbar fat. Where, in such cases, the human brain is able to approximate the edges of the hyperechoic bands by extrapolating information obtained in regions where this distinction is visible into regions where this distinction is not clear, the algorithm is not able to correctly estimate these edges. However, these manual approximations are also subject to inter- and intraobserver variability. Although our algorithm should be improved to allow more accurate extrapolation in such images, mimicking the extrapolation capabilities of the human brain is not easy. To circumvent the difficult task of identifying and implementing the "rules" for proper extrapolation, one could use machine-learning. Machine-learning has great potential for the segmentation of ONSD from ultrasound images, however, 
the performance of such algorithms is highly dependent on the quality and number of images used to train the machine-learning algorithm[27].

Besides that our algorithm removes the intra- and interobserver variability present in ONSD values due to manual assessment, it might also be used to improve the sensitivity of ONSD to changes in ICP. Because our algorithm yields ONSD values not only at the conventionally used $3 \mathrm{~mm}$ but also at a wide range of other depths, it allows us to investigate the sensitivity of ONSD to changes in ICP at multiple depths. This might give answer to the question if ONSD assessment at $3 \mathrm{~mm}$ is the best practice in all patients[28, 29]. Moreover, the automatic analysis can be used to extend the ICP-ONSD relation by investigating new metrics related to the optic nerve sheath or by combining it with other methods, such as the assessment of the optic nerve sheath pulsatility[30] determined by tracking the optic nerve sheath over multiple frames of a B-mode image.

\subsection{Conclusion}

In this study, we developed an automated optic nerve sheath diameter algorithm to estimate the ONSD from B-mode ultrasound images. Using intensity invariant asymmetry features followed by an active contour segmentation we were able to segment the optic nerve sheath diameter successfully in 39 of the 42 images. The ONSD derived with the algorithm showed good agreement with manual expert assessments. Moreover, the differences between the automatically- and manuallyderived ONSD values were within the ranges of interobserver variabilities reported in literature. Because the algorithm has no intrinsic variability it eliminates the intra- and interobserver variabilities in ONSD values associated with manual ONSD assessment. Therefore, we believe that our algorithm can be used to derive more accurate ONSD cut-off values for the detection of elevated ICP. 


\section{Appendix}

\section{A Monogenic signal}

The local phase and corresponding asymmetry features can be derived from the monogenic signal, which is the extension of the analytic signal to the $2 \mathrm{D}$ domain. To get a conceptual idea of the steps involved in the calculation of the monogenic signal we will first explain the analytic signal. For this, we based ourselves on the elaboration as presented by Bridge [20] because it offers a clear conceptually understanding of the analytic and monogenic signal without going too deep into the mathematics involved.

\section{Analytic signal}

Let us assume we have a real-valued time signal $f(t)$ and let $F(\omega)$ be its amplitude density spectrum in the frequency domain obtained by the forward Fourier transformation. Now we introduce the analytical signal $F_{a}$ by combining the Fourier spectrum $F(\omega)$ with its Hilbert transform $F_{h}(\omega)$ :

$$
\begin{aligned}
F_{a}(\omega) & =F(\omega)+i F_{h}(\omega) \\
& =F(\omega)+i(-i \operatorname{sgn}(\omega) F(\omega)) \\
& =(1+\operatorname{sgn}(\omega)) F(\omega),
\end{aligned}
$$

with

$$
\operatorname{sgn}(\omega)= \begin{cases}1, & \omega>0 \\ -1, & \omega<0 \\ 0, & \omega=0\end{cases}
$$



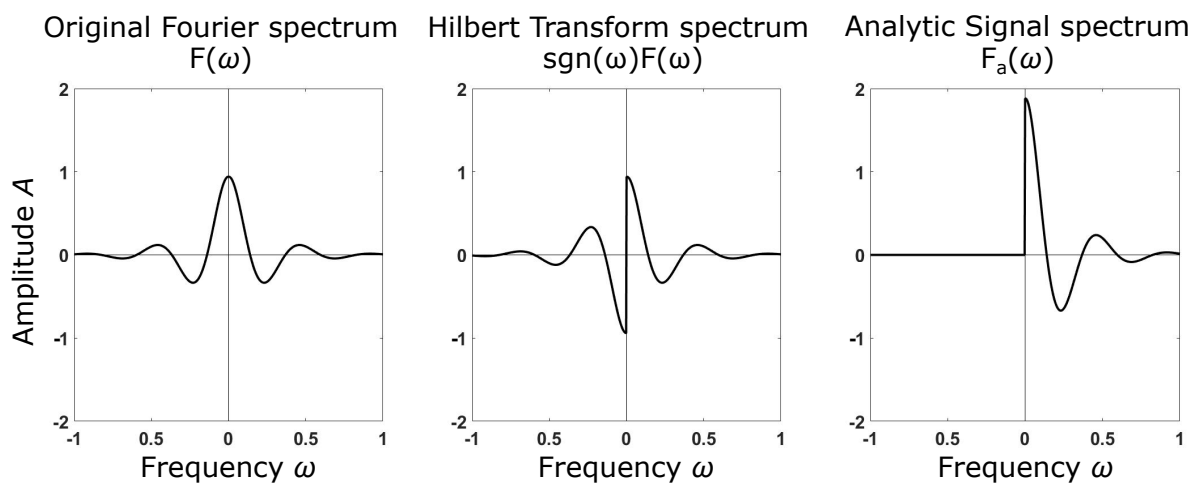

Figure 3.8: A schematic drawing of the construction of the $1 \mathrm{D}$ analytic signal. Left: The original Fourier spectrum. Middle: The Hilbert transform of the Fourier spectrum. Right: The analytic signal obtained by summing the original and the Hilbert spectrum.

This construction of the analytic signal can be seen as removing the negative frequencies of the Fourier amplitude density spectrum (see Figure 3.8). Although we constructed the analytic signal in the frequency domain, it is also possible to express the analytic signal in the time-domain: $f_{a}(t)=f(t)+i f_{h}(t)$ with $f_{h}(t)=\mathcal{H}(f(t))$, where $\mathcal{H}$ is the Hilbert transform. Moreover, we can switch between $F_{a}(\omega)$ and $f_{a}(t)$ using the inverse Fourier transform or the Fourier transform.

To control the frequencies that are included in the calculation of the monogenic signal, we add a bandpass filter operation to Equation 3.9:

$$
F_{a}(\omega)=(1+\operatorname{sgn}(\omega)) G(\omega) F(\omega)
$$

A popular bandpass filter is the log-Gabor function (see Figure 3.9) because it can be constructed with arbitrary width while still maintaining a zero-gain at $\omega=0$ $\mathrm{rad} / \mathrm{s}$ (the DC component)[18-20]. The log-Gabor filter is given by:

$$
G(\omega)=\exp \left(-\frac{\left(\log \left(\frac{|\omega|}{\omega_{0}}\right)\right)^{2}}{2\left(\log \left(\sigma_{0}\right)\right)^{2}}\right) .
$$

Here, $|\cdot|$ is the $L^{2}$-norm, $\omega_{0}$ is the center frequency that effects the scale of the features that are detected within the signal, and $\sigma_{0}$ the shape parameter that controls the bandwidth of the filter.

From Equation 3.11 it becomes clear that filtering our image with the log-Gabor filter $(G(\omega) F(\omega))$ and then calculating the analytic signal of this part (multiplication with $(1+\operatorname{sgn}(\omega)))$ is mathematically equivalent to first deriving the analytic response 

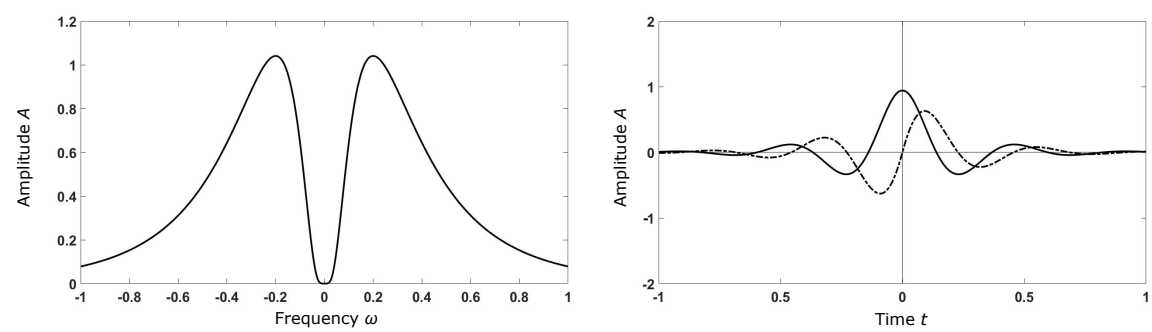

Figure 3.9: Left: an example of a Log-Gabor filter in the frequency domain. Right: Schematic representation of the analtic filter response of a log-Gabor filter in the time domain. The solid line denotes the (even) symmetric response $(f(t))$, whereas the dashed lines denotes the (odd) symmetric response $\left(f_{h}(t)\right)$. Note the shift of $\pi / 2$ to the right of the odd filter with respect to the even filter.

of the filter $((1+\operatorname{sgn}(\omega)) G(\omega))$ and then applying this "analytic filter" to our original image (multiplication with $F(\omega)$ ). We apply the second method as it allows for a more intuitive explanation of the filter responses.

Transforming the "analytic filter" that is defined in the frequency domain back to the time-domain, gives a complex-valued signal that consists of two parts (see Figure 3.9). These two parts of the "analytic filter" have some interesting properties. As we convolute these filters over a signal that contains a peak or trough, the symmetric filter (real part) will give a response that is positive on the left side of the peak, maximum exactly on the peak, and positive on the right side of the peak, resulting in a net response that is positive. The asymmetric filter, on the other hand, has a response that is negative on the left side of the peak, zero on the peak, and positive on the right side of the peak, resulting in a net value close to zero. The relation between these two filter responses can be captured in the local amplitude and local phase of the signal:

$$
\begin{aligned}
& A(t)=\sqrt{f(t)^{2}+f_{h}(t)^{2}}, \\
& \phi(t)=\arctan \left(\frac{f_{h}(t)}{f(t)}\right),
\end{aligned}
$$

Especially the local phase can be useful in our application because it incorporates the two different responses of the "analytic filter" and can therefore be used to detect edge-like structures in an image. 


\section{Monogenic signal}

Now lets extend the analytic signal to 2D and apply it to images. Hereto, we use the monogenic signal, a generalisation of the analytic signal derived by Felsberg et al. [31]. Lets assume we have an image $\mathrm{f}(\mathbf{x})$ with $\mathbf{x}=(x, y)^{T}$ the coordinates of the pixels and $F(\boldsymbol{\omega})$ being its representation in the frequency-domain, where $\boldsymbol{\omega}=\left(\omega_{x}, \omega_{y}\right)^{T}$ are the frequencies in the two image directions. The first step is the calculation of the analytical filter. Hereto, we need to calculate the odd part of the filter. However, because we our now in $2 \mathrm{D}$ instead of $1 \mathrm{D}$, we need to calculate two odd parts, i.e. one for each image direction. Moreover, we have to use a generalisation of the Hilbert transform known as the Riesz transform. Similar as in the 1D example we choose the (even) log-Gabor filter:

$$
G_{e}(\boldsymbol{\omega})=\exp \left(-\frac{\left(\log \left(\frac{|\boldsymbol{\omega}|}{\omega_{0}}\right)\right)^{2}}{2\left(\log \left(\sigma_{0}\right)\right)^{2}}\right),
$$

where $\omega_{0}$ and $\sigma_{0}$ are still the center frequency and the shape parameter that controls the width of the filter, respectively. The two odd parts of the "analytic filter" are generated by:

$$
\begin{aligned}
& G_{o_{x}}(\boldsymbol{\omega})= \begin{cases}i \frac{\omega_{x}}{|\boldsymbol{\omega}|} G(\boldsymbol{\omega}), & \boldsymbol{\omega} \neq 0, \\
0, & \boldsymbol{\omega}=0,\end{cases} \\
& G_{o_{y}}(\boldsymbol{\omega})= \begin{cases}i \frac{\omega_{y}}{|\boldsymbol{\omega}|} G(\boldsymbol{\omega}), & \boldsymbol{\omega} \neq 0, \\
0, & \boldsymbol{\omega}=0 .\end{cases}
\end{aligned}
$$

Next, we filter the image $f(\mathbf{x})$ with our three 'analytic filters' and transform the result back to the time-domain, i.e. spatial-domain $\mathbf{x}$, to obtain the monogenic signal $\mathbf{f}(\mathbf{x})$ of the image:

$$
\mathbf{f}_{m}(\mathbf{x})=\left[\begin{array}{c}
f_{e}(\mathbf{x}) \\
f_{o_{x}}(\mathbf{x}) \\
f_{o_{y}}(\mathbf{x})
\end{array}\right]
$$

The two different odd parts of the monogenic signal contain image changes that have wavelengths close to $\lambda_{0}=\frac{2 \pi}{\omega_{0}}$ each in a different image direction.

Similar as in the $1 \mathrm{D}$ case we can use the local phase to detect symmetric or asymmetric edges (peaks and troughs) in an image. However, edges in an image might not be purely symmetrical or asymmetric. It might therefore be useful to quantify to what extend the region surrounding a pixel is symmetric/asymmetric. In addition, it can be useful to calculate the monogenic signal and the corresponding features over multiple wavelengths $\lambda$ instead of just one because, in contrast to noise, structures 
of interest often manifest themselves within multiple wavelengths. Therefore, we use Kovesi's feature asymmetry over multiple wavelengths:

$$
R(\mathbf{x})=\sum_{i} \frac{\left\lfloor\left|f_{o, \lambda_{i}}(\mathbf{x})\right|-\left|f_{e, \lambda_{i}}(\mathbf{x})\right|-T\right\rfloor}{A_{\lambda_{i}}(\mathbf{x})+\epsilon} .
$$

Here, $\lambda_{i}$ is the set of wavelengths used to calculate the monogenic signal, $T$ is a threshold that determines the sensitivity which was set to 0.18 [20]. In addition, $\lfloor\cdot\rfloor$ is a shorthand indicating that values smaller than zero are set to zero, and $\epsilon$ is a small number that prevents division by zero. Finally, the term $f_{o, \lambda_{i}}(\mathbf{x})$ is the combined odd component given by:

$$
f_{o, \lambda_{i}}(\mathbf{x})=\sqrt{f_{o_{x}, \lambda_{i}}(\mathbf{x})^{2}+f_{o_{y}, \lambda_{i}}(\mathbf{x})^{2}}
$$

and, $A_{\lambda_{i}}(\mathbf{x})$ is the amplitude of the combined odd component and the even function:

$$
A_{\lambda_{i}}(\mathbf{x})=\sqrt{f_{e, \lambda_{i}}(\mathbf{x})^{2}+f_{o, \lambda_{i}}(\mathbf{x})^{2}} .
$$

The feature asymmetry $R(\mathbf{x})$ has values between o and 1 where 1 indicates that the surrounding region is very asymmetric and o indicates that the region is very symmetric. It is useful to know the direction of asymmetry around an edge, e.g. does the intensity change from low to high, or from high to low. Therefore, we choose to retain the sign of the edge in our asymmetry measure. This is known as the signed feature asymmetry and can be calculated as follows[20]:

$$
\hat{R}_{j}(\mathbf{x})=\sum_{i} \frac{\left\lfloor\left|f_{o, \lambda_{i}}(\mathbf{x})\right|-\left|f_{e, \lambda_{i}}(\mathbf{x})\right|-T\right\rfloor}{A_{\lambda_{i}}(\mathbf{x})+\epsilon} \frac{f_{o_{j}, \lambda_{i}}(\mathbf{x})}{\left|f_{o, \lambda_{i}}(\mathbf{x})\right|} .
$$

Here, $j$ denotes either the first or second odd component in respectively, the $x$ and $y$-direction. The values $\hat{R}_{j}(\mathbf{x})$ lie between -1 and +1 where values close to -1 or 1 correspond to edges that are respectively, troughs or peaks. 


\section{References}

1. Lochner, P., Fassbender, K., Lesmeister, M. et al. Ocular ultrasound for monitoring pseudotumor cerebri syndrome. eng. Journal of neurology 265, 356-361 (2018).

2. Moretti, R., Pizzi, B., Cassini, F. \& Vivaldi, N. Reliability of optic nerve ultrasound for the evaluation of patients with spontaneous intracranial hemorrhage. eng. Neurocritical Care 11, 406-410 (2009).

3. Lochner, P., Cantello, R., Fassbender, K. et al. Longitudinal Assessment of Transorbital Sonography , Visual Acuity , and Biomarkers for Inflammation and Axonal Injury in Optic Neuritis. 2o17, 4-9 (2017).

4. Hansen, H. C. \& Helmke, K. Validation of the optic nerve sheath response to changing cerebrospinal fluid pressure: Ultrasound findings during intrathecal infusion tests. Journal of Neurosurgery $\mathbf{8 7}$, 34-40 (1997).

5. Geeraerts, T., Merceron, S., Benhamou, D. et al. Non-invasive assessment of intracranial pressure using ocular sonography in neurocritical care patients. eng. Intensive Care Medicine 34, 2062-2067 (2008).

6. Toscano, M., Spadetta, G., Pulitano, P. et al. Optic Nerve Sheath Diameter Ultrasound Evaluation in Intensive Care Unit: Possible Role and Clinical Aspects in Neurological Critical Patients' Daily Monitoring. BioMed Research International 2017, 1-7 (2017).

7. Soldatos, T., Karakitsos, D., Chatzimichail, K., Papathanasiou, M., Gouliamos, A. \& Karabinis, A. Optic nerve sonography in the diagnostic evaluation of adult brain injury. Critical Care 12, 1-7 (2008).

8. Hansen, H. C. \& Helmke, K. The subarachnoid space surrounding the optic nerves. An ultrasound study of the optic nerve sheath. Surgical and radiologic anatomy : SRA 18, 323-8 (1996).

9. Bäuerle, J., Lochner, P., Kaps, M. \& Nedelmann, M. Intra- and Interobsever Reliability of Sonographic Assessment of the Optic Nerve Sheath Diameter in Healthy Adults. Journal of Neuroimaging 22, 42-45 (2012).

10. Lochner, P., Coppo, L., Cantello, R. et al. Intra- and interobserver reliability of transorbital sonographic assessment of the optic nerve sheath diameter and optic nerve diameter in healthy adults. Journal of Ultrasound 19, 41-45 (2016).

11. Dubourg, J., Javouhey, E., Geeraerts, T., Messerer, M. \& Kassai, B. Ultrasonography of optic nerve sheath diameter for detection of raised intracranial pressure: A systematic review and metaanalysis. eng. Intensive Care Medicine 37, 1059-1068 (2011).

12. Wang, L.-J. J., Yao, Y., Feng, L.-S. S. et al. Noninvasive and quantitative intracranial pressure estimation using ultrasonographic measurement of optic nerve sheath diameter. eng. Scientific Reports 7, 1-7 (2017).

13. Pichamuthu, K. K. \& Prithishkumar, I. J. Appearance of the Optic Nerve Sheath Diameter (ONSD) using Higher Frequency Linear Probes in Detection and Monitoring of Raised Intracranial Pressures-A Cadaveric Study. Journal of Clinical and Diagnostic Research, 10-13 (2019).

14. Bloria, S., Bloria, P. \& Luthra, A. Is it the time to standardize the procedure of ultrasound guided optic nerve sheath diameter measurement? Saudi Journal of Anaesthesia 13, 255 (2019).

15. Gerber, S., Jallais, M., Greer, H. et al. Imaging for Patient-Customized Simulations and Systems for Point-of-Care Ultrasound. 10549 (2017).

16. Soroushmehr, R., Rajajee, K., Williamson, C. et al. Automated Optic Nerve Sheath Diameter Measurement Using Super-pixel Analysis. 2019 41st Annual International Conference of the IEEE Engineering in Medicine and Biology Society (EMBC), 2793-2796 (2019). 
17. Meiburger, K. M., Naldi, A., Michielli, N. et al. Automatic Optic Nerve Measurement: A New Tool to Standardize Optic Nerve Assessment in Ultrasound B-Mode Images. Ultrasound in Medicine and Biology 46, 1533-1544 (2020).

18. Rackham, T. M., Rueda, S., Knight, C. L. \& Noble, J. A. Ultrasound image segmentation using feature asymmetry and shape guided live wire. Medical Imaging 2013: Image Processing 8669, 86690 P (2013).

19. Mulet-Parada, M. \& Noble, J. A. 2D+T acoustic boundary detection in echocardiography. Lecture Notes in Computer Science (including subseries Lecture Notes in Artificial Intelligence and Lecture Notes in Bioinformatics) 1496, 807-813 (1998).

20. Bridge, C. P. Introduction To The Monogenic Signal, 1-21 (2017).

21. Lankton, S. \& Tannenbaum, A. R. Localizing Region-Based Active Contours. \{IEEE\} Trans. Image Processing 17, 2029-2039 (2008).

22. Pang, J. Lozalized Active Contour 2020.

23. Chan, T. F. \& Vese, L. A. Active contours without edges. IEEE Transactions on Image Processing 10, 266-277 (2001).

24. Bäuerle, J., Schuchardt, F., Schroeder, L. et al. Reproducibility and accuracy of optic nerve sheath diameter assessment using ultrasound compared to magnetic resonance imaging. BMC neurology 13, 187 (2013).

25. Ballantyne, S. A., O’Neill, G., Hamilton, R. \& Hollman, A. S. Observer variation in the sonographic measurement of optic nerve sheath diameter in normal adults. European Journal of Ultrasound 15, 145-149 (2002).

26. Copetti, R. \& Cattarossi, L. Optic nerve ultrasound: Artifacts and real images. Intensive Care Medicine 35, 1488-1489 (2009).

27. Cantisani, V., Grani, G., Tovoli, F., Piscaglia, F. \& Catalano, C. Artificial Intelligence: What is it and How can it expand the ultrasoun. Ultraschall in der Medizin (Stuttgart, Germany : 1980) 41, 356-360 (2020).

28. Vaiman, M., Sigal, T., Kimiagar, I. \& Bekerman, I. Intracranial pressure assessment in traumatic head injury with hemorrhage via optic nerve sheath diameter. Journal of Neurotrauma 33, 2147-2153 (2016).

29. Vaiman, M., Gottlieb, P. \& Bekerman, I. Quantitative relations between the eyeball, the optic nerve, and the optic canal important for intracranial pressure monitoring. Head and Face Medicine 10, 1-6 (2014).

30. Padayachy, L., Brekken, R., Fieggen, G. \& Selbekk, T. Pulsatile dynamics of the optic nerve sheath and intracranial pressure: An exploratory in vivo investigation. Neurosurgery 79, 100-107 (2016).

31. Felsberg, M. \& Sommer, G. The monogenic signal. IEEE Transactions on Signal Processing 49, 3136-3144 (2001). 

Part |

Estimation of cerebral boundary conditions 



\section{The Role of Inter-Subject Variations on Wall Shear Stress Indices of Intracranial Aneurysms}

Based on: Stevens, R.R.F., Donders, W., Quicken, S., van de Vosse, F.N., Mess, W.H., Delhaas, T. \& Huberts, W. The Role of One-Dimensional Model-Generated Inter-Subject Variations in Systemic Properties on Wall Shear Stress Indices of Intracranial Aneurysms IEEE Trans Biomed Eng. 2020 Apr;67(4):1030-1039 . 


\begin{abstract}
Variations in systemic properties of the arterial tree, such as ageing-induced vessel stiffness, can alter the shape of pressure and flow waveforms. As a consequence, the hemodynamics around a cerebral aneurysm change, and therefore also the corresponding in- and outlet boundary conditions (BCs) used for 3D calculations of hemodynamic indices. In this study, we investigated the effects of variations in systemic properties on wall shear stress (WSS) indices of a cerebral aneurysm. We created a virtual patient database by varying systemic properties within physiological ranges. BCs for 3D-CFD simulations were derived using a pulse wave propagation model for each realisation of the virtual database. WSS indices were derived from the 3D simulations and their variabilities quantified. Variations in BCs, caused by changes in systemic properties, yielded variabilities in the WSS indices that were of the same order of magnitude as differences in these WSS indices between ruptured and unruptured aneurysms. Sensitivity analysis showed that the systemic properties impacted both in- and outlet BCs simultaneously and altered the WSS indices. We conclude that the influence of variations in patient-specific systemic properties on WSS indices should be evaluated when using WSS indices in multidisciplinary rupture risk prediction models.
\end{abstract}




\subsection{Introduction}

W

E I GH ING the impact of cerebral aneurysm rupture against the risk of treatment is a dilemma for clinicians. Therefore, various studies have attempted to find indicators of aneurysm rupture risk. Although, patient factors[1], morphological parameters [2-5] and hemodynamic indices derived from computational fluid dynamics (CFD) simulations [2, 3, 6-8], showed to be correlated with aneurysm rupture risk, none of these risk indicators can accurately stratify aneurysm rupture risk independently. As advised by guidelines[9], researchers therefore started to combine patient- and aneurysm features with the hemodynamic metrics[10-12] in, for example, regression models. Such multidisciplinary models have shown promising results in predicting rupture risk[13, 14]. However, the accuracy of the rupture prediction might be compromised by uncertainties present within the risk indicators used. This is especially true for the hemodynamic indices that result from CFD simulations because they are intrinsically compromised by uncertainties due to amongst others, aneurysm segmentation and inaccurate inand outlet boundary conditions (BCs). The effect of inlet $\mathrm{BCs}$ on rupture risk prediction has already been shown by, for example, Sarrami-Foroushani et al. [15]. In their study, they found that variations in inlet BCs changed the rupture outcome predictions of their logistic regression model (using both morphological and hemodynamic indices as regressors) in approximately $20 \%$ of the cases. Though BCs are largely patient-specific and dependent on the physiological envelope of the patient (e.g. daily activity), the assessment of patient-specific in- and outlet BCs is rarely performed in clinical practice and even impossible in a significant number of cases. Consequently, most researchers resort to typical (patient-generic) inlet conditions and scale, for example, the resulting hemodynamic indices to the wall shear stress at the inlet to minimize patient differences in BCs when comparing results. At the outlet often non-physiological BCs are chosen that are uncorrelated with the inlet BCs and do not take into account the differences in waveforms that might result from differences in the peripheral and systemic vasculature of the patient [16]. Marzo et al. [16] have shown that patient-generic 1D pulse wave propagation models (PWPM) that simulate the pressure and flow at the in- and outlets[17], are the best alternative in the absence of patient-specific BCs. PWPMs have been validated against clinical measurements[18], and have been used to investigate the necessity of patient-specific modeling $[16,17,19,20]$. Using a PWPM to assess the BCs automatically results in correlated in- and outlet BCs while simultaneously considering the systemic and peripheral vasculature even when the aneurysm domain contains more than one outlet[21]. An additional advantage of using a PWPM is the possibility to examine the effect of intra- and inter-subject variations in systemic vascular properties like aortic inflow and ageing-induced vessel stiffness, on the in- 
and outlet $\mathrm{BCs}[22]$. In this study, we aim to investigate the role of such variations in systemic properties on four commonly used Wall Shear Stress (WSS) indices calculated with 3D-CFD. Specifically, we examine the influence of mean arterial pressure, mean aortic inflow, total arterial compliance, mean cerebral blood flow, and age-related vessel stiffness and radius changes. Hereto, we created a virtual database of sets of systemic properties. BCs for 3D-CFD simulations were derived for each virtual patient using a PWPM and WSS indices were calculated from the CFD results. Global variance-based sensitivity analysis[23] was performed to assess, how variation in each systemic property impacts the variation within the WSS indices. Our approach might help to identify systemic properties that can improve the aneurysm rupture risk predictions done by multidisciplinary models.

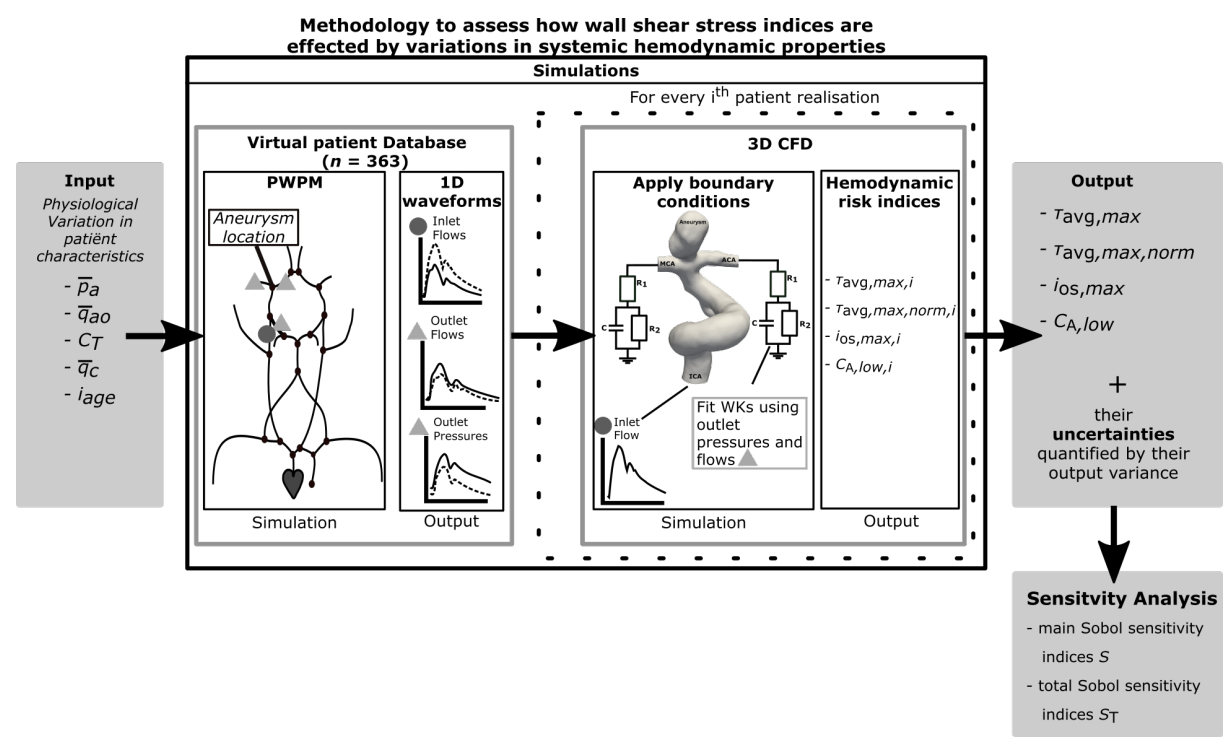

Figure 4.1: Overview of the methodology used to quantify the effect of changes in systemic properties on wall shear stress rupture risk indices of an aneurysm. A virtual database is created by varying five systemic properties within their physiological ranges. 1D pulse wave propagation model simulations are performed for each realisation of the virtual database, and pressure and flow waveforms are extracted at locations corresponding to the in- and outlets of the 3D aneurysm domain and used to fit boundary conditions for the 3D-CFD simulations. Four commonly used wall shear stress indices are then computed from the CFD simulations. Global variance-based sensitivity analysis is performed to quantify the contribution of each of the systemic properties to the variance present within the wall shear stress indices. 
Table 4.1: Baseline values and ranges of the systemic properties used to generate the virtual database.

\begin{tabular}{lllll}
\hline Description & Symbol & Unit & Baseline & Range \\
\hline Mean arterial pressure & $\bar{p}_{\mathrm{a}}$ & $10^{3} \mathrm{~Pa}$ & 11.7 & $9 \cdot 33^{-14}[26-28]$ \\
Mean aortic inflow & $\bar{q}_{\mathrm{ao}}$ & $10^{-5} \mathrm{~m}^{3} \mathrm{~s}^{-1}$ & 8.33 & $6.67-10.0[22,29,30]$ \\
Total arterial compliance & $C_{\mathrm{t}}$ & $10^{-9} \mathrm{~m}^{3} \mathrm{~Pa}^{-1}$ & 11.2 & $7 \cdot 5^{-15}[31]$ \\
Mean cerebral blood flow & $\bar{q}_{\mathrm{c}}$ & $10^{-5} \mathrm{~m}^{3} \mathrm{~s}^{-1}$ & 1.17 & $1-1.33[32]$ \\
Age index & $i_{\text {age }}$ & - & 0 & $0-1$ \\
\hline
\end{tabular}

\subsection{Materials and Methods}

In this section we describe our approach following the outline as depicted in Figure 4.1. First, we elaborate on the systemic properties which were varied to create the virtual database. Next, we describe the PWPM and the way the systemic properties are incorporated in the model. Subsequently, we present details of the 3D-CFD simulations and introduce the WSS indices. Finally, we discuss the sensitivity analysis.

\subsubsection{Virtual patient database}

A virtual patient database was created that consisted of multiple realisations of different values for mean (time-averaged) arterial pressure $\bar{p}_{a}(\mathrm{~Pa})$, mean aortic inflow $\bar{q}_{a o}\left(\mathrm{~m}^{3} \mathrm{~s}^{-1}\right)$, total arterial compliance $C_{T}\left(\mathrm{~m}^{3} \mathrm{~Pa}^{-1}\right)$, mean cerebral blood flow $\bar{q}_{c}\left(\mathrm{~m}^{3} \mathrm{~s}^{-1}\right)$, and vascular age index $i_{\text {age }}(-)$. We refer the reader to Method section B for implementation details. The systemic properties $\bar{p}_{a}, \bar{q}_{a o}, \bar{q}_{c}$ and $C_{T}$ were used to assess the outflow BCs for the $1 \mathrm{D}$ model. In addition, $\bar{q}_{a o}$ was used to scale the inflow waveform prescribed at the inlet of the $1 \mathrm{D}$ domain. Moreover, the vascular age index $i_{\text {age }}$ was used to mimic the effect of increasing arterial wall radii and stiffnesses with age from approximately 25 to 70 years[24]. The systemic properties were uniformly sampled within physiological ranges using the low discrepancy Sobol sequence[25] via the built-in Matlab function sobolset. Based on an a priori estimation (see Section 4.2.5) a database of 363 samples was created. Each sample from the virtual database represents one virtual patient. Baseline values and corresponding ranges of the systemic properties are listed in Table 4.1 and Table 4.2. Details about the implementation of these parameters into the model will be elaborated in the next section. 
Table 4.2: Changes of the vessel radii and stiffness (Young's Modulus) corresponding to changes in age index

\begin{tabular}{lll}
\hline Description & Symbol & Change in \% \\
\hline Radius (proximal vessels)* & $\mathrm{r}_{\text {prox }}$ & $0-30[26,33]$ \\
Radius (distal vessels)** & $\mathrm{r}_{\text {dist }}$ & $0-20[27,34]$ \\
Youngs Modulus (proximal vessels) & $\mathrm{E}_{\text {prox }}$ & $0-625[24]$ \\
Youngs Modulus (distal vessels) & $\mathrm{E}_{\text {dist }}$ & $0-250[24]$ \\
\hline${ }^{*}$ The aortic vessels are considered proximal & \\
${ }^{* *}$ All vessels except the aortic vessels are considered distal & \\
\end{tabular}

\subsubsection{Pulse Wave Propagation Model}

The arterial domain of the 1D PWPM consisted of the aorta, the main arteries of the arms, and the brain vasculature with a complete Circle of Willis. Details about the arterial domain such as vessel radii and Young's moduli, can be found in Mulder et al. [17]. The arterial domain was divided in elements. Each element represented the local relation between pressure, flow, and vessel cross-sectional area[35]. The $1 \mathrm{D}$ wave propagation equations of mass and momentum conservation were obtained by integrating the incompressible Navier-Stokes equations over the cross-sectional area of the blood vessel, hereby assuming only flow in axial direction. The thin-walled tube formulation was used to describe the pressure-area relation, hereby assuming elastic behavior of the wall. Truncated vessels were closed using 0D three-element windkessel models that consisted of two resistances $R_{1}$ and $R_{2}$, and a compliance $C$, which mimic the effect of the peripheral impedance distal to the truncated vessels. The total terminal resistance of each truncated vessel $t$, was calculated according to[17]:

$$
R_{\mathrm{p}, t}=\frac{R_{\mathrm{T}}}{q_{\text {frac }}} \frac{\sum_{i=1}^{N_{\mathrm{t}}} a_{0, i}^{3}}{a_{0, t}^{3}},
$$

with $N_{\mathrm{t}}$ the total number of vessels within the corresponding body compartment and $a_{0, t}$ the vessel radius of the $t^{\text {th }}$ vessel[17]. Total peripheral resistance of the PWPM, $R_{\mathrm{T}}$, was calculated using input parameters $\bar{p}_{\mathrm{a}}$ and $\bar{q}_{\mathrm{ao}}$, according to $R_{\mathrm{T}}=\bar{p}_{\mathrm{a}} / \bar{q}_{\mathrm{ao}}$. The parameter $q_{\text {frac }}$ denotes the flow fraction of the total cardiac output towards the body part. The cerebral flow fraction was calculated using mean cerebral blood flow $\bar{q}_{\mathrm{c}}$, i.e. $q_{\mathrm{frac}, b}=\bar{q}_{\mathrm{c}} / \bar{q}_{\mathrm{ao}}$, whereas flow fractions towards each arm were set to $5 \%$ of the cardiac output $[18,36,37]$. The remaining flow was assigned to the lower body, i.e. descending aorta.

The windkessel resistances $R_{1, t}$ and $R_{2, t}$, for each truncated vessel were calculated using $R_{\mathrm{p}, t}=R_{1, t}+R_{2, t}$. To minimise non-physiological reflections for high fre- 
quencies, the resistance $R_{1, t}$ was chosen as the characteristic impedance of the corresponding branch $t$. The compliance of the peripheral beds, $C_{0 \mathrm{D}}$, was calculated as the difference between the total compliance of the human body and the compliance of the $1 \mathrm{D}$ domain. This compliance $C_{0 \mathrm{D}}$, was distributed according to $C_{\mathrm{p}, t}=C_{0 \mathrm{D}} * R_{\mathrm{T}} / R_{\mathrm{p}, t}[17]$. Lastly, a generic aortic flow waveform with a cycle time of is was prescribed at the aorta[38]. This waveform was scaled using the corresponding $\bar{q}_{\mathrm{ao}}$.

\subsubsection{D Simulation}

The $3 \mathrm{D}$ computational domain of the aneurysm (Figure 4.2) was taken from Mulder et al. [17]. The 3D domain consisted of a section of the right internal carotid artery (ICA) that splits into the right middle cerebral artery (MCA) and right anterior cerebral artery (ACA). In addition, the right posterior communicating artery (PCoA) branched off from the right ICA before the bifurcation. The aneurysm was located at the tip of the bifurcation of the ICA, MCA, and ACA.

We used an in-house finite element method (TFEM[39]) to solve the Navier-Stokes equations for an incompressible Newtonian fluid. Time discretization was achieved with a second order backward difference scheme. Blood density and viscosity were set to $\rho=1050 \mathrm{~kg} \mathrm{~m}^{-3}$ and $\eta=3 \cdot 5 \cdot 10^{-3} \mathrm{~Pa}$, respectively. The computational domain was meshed using quadratic tetrahedral Taylor-Hood elements. In addition, we used the streamline upwind technique[40], to stabilize our simulations. This technique, made it possible to use larger elements and timesteps, which reduced our computational time tremendously. The mesh density was approximately 56 elements per $\mathrm{mm}^{3}$. A mesh convergence study showed stable and mesh-independent results for the chosen mesh density. Vessel walls were assumed to be rigid and a no-slip boundary condition was imposed. ICA flow waveforms calculated with the PWPM were applied at the inlet of the $3 \mathrm{D}$ mesh. Local output impedances were calculated using 1D outlet pressure and flow waveforms obtained from the PWPM. These impedances were used to fit windkessels for each $3 \mathrm{D}$ outlet. Hereto, $R_{1}$, $R_{2}$, and $C$ were chosen such that the windkessels' impedance spectra amplitudes resembled the load impedances as close as possible. The obtained boundary conditions were used to perform 3D-CFD simulations for every single realisation of the virtual database.

\subsubsection{Wall shear stress-based rupture risk indices}

Four commonly used WSS indices were computed from the CFD simulations using MATLAB R2015a (The MathWorks, Inc., Natick, Massachusetts, United States). First, we calculated the maximum value of the time-averaged WSS magnitude 


\section{Inlet Outlet}

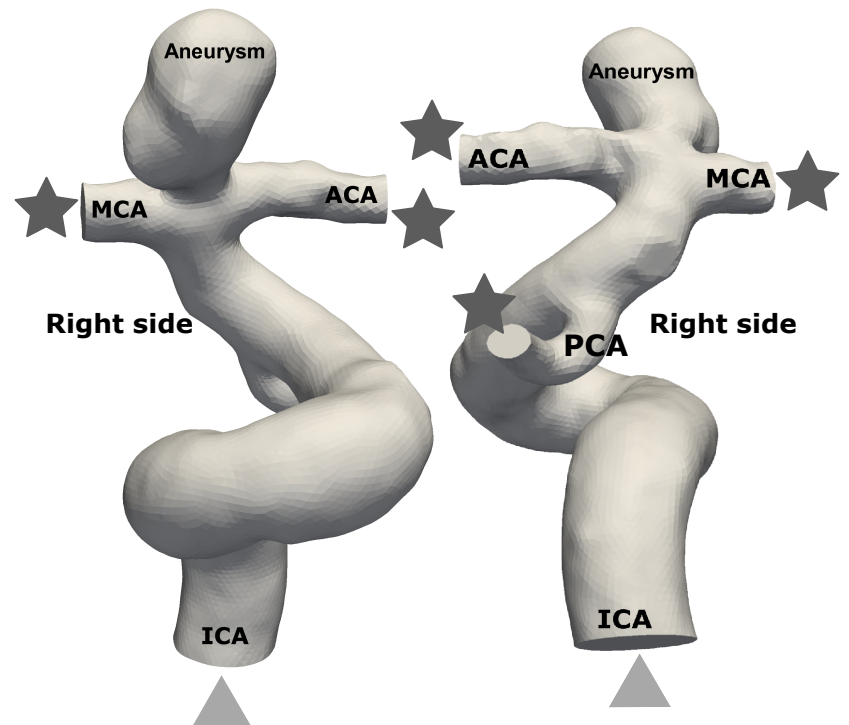

Figure 4.2: Anterior (left) and posterior view (right) of the surface mesh. The cerebral aneurysm is located at the bifurcation of the right internal carotid artery (ICA) into the right anterior cerebral artery (ACA) and right middle cerebral artery (MCA). In the posterior view (right) the right posterior communicating artery (PCoA) is visible[17].

$\tau_{\mathrm{avg}, \max }$, within the complete boundary of the aneurysm domain $\Omega$ :

$$
\tau_{\mathrm{avg}, \max }=\max \left(\tau_{\mathrm{avg}}(\vec{\Omega})\right)
$$

with:

$$
\tau_{\text {avg }}(\vec{\Omega})=\frac{1}{T} \int_{t=0}^{t=T}\|\vec{\tau}(\vec{\Omega}, t)\| d t,
$$

the time-averaged WSS magnitude, $\|.$.$\| the l^{2}$ norm, cycle time $T(\mathrm{~s})$, and $\vec{\Omega}$ the vector containing the spatial coordinates of the aneurysm wall.

Normalised maximum time-averaged WSS magnitude $\tau_{\text {avg, max }}^{*}$, was computed by normalising $\tau_{\text {avg, } \max }$ with the spatially averaged WSS magnitude $\bar{\tau}_{a v g}$ :

$$
\tau_{\mathrm{avg}, \max }^{*}=\frac{\max \left(\tau_{\mathrm{avg}}(\vec{\Omega})\right)}{\bar{\tau}_{\text {avg }}} .
$$


Furthermore, the maximum value of the oscillatory shear index (OSI) was calculated. The maximum OSI, $i_{\mathrm{os}, \max }$, is given by:

$$
i_{\mathrm{os}, \max }=\max \left(i_{\mathrm{os}}(\vec{\Omega})\right),
$$

with:

$$
i_{\mathrm{os}}(\vec{\Omega})=\frac{1}{2}\left(1-\frac{\left\|\int_{t=0}^{t=T} \vec{\tau}(\vec{\Omega}, t) d t\right\|}{\int_{t=0}^{t=T}\|\vec{\tau}(\vec{\Omega}, t)\| d t}\right),
$$

Finally, the percentage aneurysm area with a low $(<0.5 \mathrm{~Pa})$ time-averaged WSS, $c_{\mathrm{A}, \text { low }}$, was calculated. The $95^{\text {th }}$ percentile intervals of the WSS indices were used to calculate the coefficient of variation, i.e. $C_{\mathrm{v}}=\frac{\sigma}{\mu}$, with $\sigma$ an $\mu$ the $2.5 \%-97.5 \%$ percentile range and $50 \%$ percentile, respectively. This coefficient was used to quantify the effect of variations in systemic properties on the WSS indices and to allow comparison with literature.

Lambert azimuthal equal-area projections were used to visualise the spatial distributions of $\tau_{\mathrm{avg}, \max }$ and $i_{\mathrm{os}, \max }$ on the aneurysm dome. This projection method maps the entire aneurysm dome to a tangent $2 \mathrm{D}$ plane so that all areas on the dome are accurately represented in size on the 2D projection (Figure 4.3). We plotted the spatial distributions of the median values of both $\tau_{\text {avg, } \max }$ and $O S I$. These distributions were determined by calculating the median value over all simulations for every point on the aneurysm dome. We also visualised local variations of $\tau_{\text {avg, } \max }$ and $i_{\mathrm{os}, \max }$. Variations were determined by taking $\tau_{\mathrm{avg}, \max }$ and $i_{\mathrm{os}, \max }$ values of all simulations and calculating the ranges of the $95^{\text {th }}$ percentile interval for every point on the aneurysm surface.

\subsubsection{Uncertainty quantification and sensitivity analysis}

A global variance-based sensitivity analysis[41] was performed to apportion each fraction of the total variance present within the WSS indices to the individual contribution of each single systemic properties (main effect) or their contributions in combination with other systemic properties (interaction effects) [42]. The direct contribution of each parameter $X_{\mathrm{i}}$ to the total output variance is quantified by the main Sobol sensitivity index $S_{\mathrm{i}}$, which represents the expected reduction in output variance that results if parameter $X_{\mathrm{i}}$ would have been known exactly. The contribution of all fractions of the total variance involving parameter $X_{\mathrm{i}}$, is quantified by the total Sobol sensitivity index $S_{\mathrm{T}}$. The total index resembles the expected output variance that remains in case all parameters except $X_{\mathrm{i}}$ would have been known exactly. To estimate the sensitivity indices, the adaptive generalised 


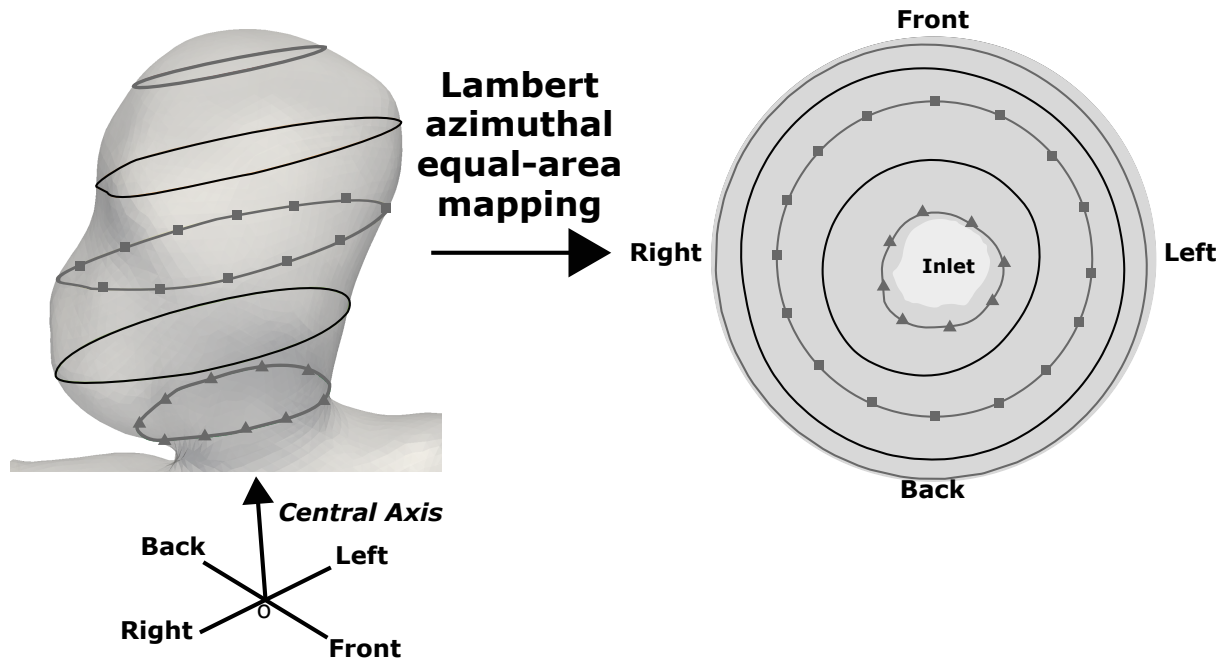

Figure 4.3: Left: detail of the intracranial aneurysm. At the bottom the coordinate system for the equal-area mapping is shown. The $\mathrm{O}$ denotes the origin of the system of axis and is placed at the inlet. The grey rings are equally spaced along the normal direction of the central axis The light grey ring with triangles corresponds with the aneurysm neck. Right: Lambert azimuthal equal-area plot of the aneurysm surface. The right plot is obtained by looking into the aneurysm from the inlet along the central axis, and unfolding the aneurysm in a circular manner.

polynomial chaos expansion (agPCE) method (described by Quicken et al. [23]) was used. The agPCE method was used to expand the output $Y$ into a set of orthogonal polynomials that depends on the stochastic input:

$$
Y_{\mathrm{i}}=f(\vec{X}) \approx \sum_{e=0}^{N_{\mathrm{p}}} c_{\mathrm{e}} \Phi_{\mathrm{e}}(\vec{X}) .
$$

Here, $\Phi_{\mathrm{e}}$ are the orthogonal multivariate Legendre polynomials and $c_{\mathrm{e}}$ the expansion coefficients which were estimated using least squares regression. The parameter $Y_{\mathrm{i}}$ denotes one of the four WSS indices (model outputs), and $\vec{X}$ denotes the five systemic properties (model input parameters). Furthermore, $N_{\mathrm{p}}$ indicates the total number of included polynomials. The polynomial expansion was constructed by adaptively adding or removing polynomials. Polynomials that significantly increased the proportion of the output variance that could be explained by the metamodel $R^{2}>\varepsilon_{1}$ were included, whereas polynomials that did significantly decrease this proportion $R^{2}<\varepsilon_{2}$ were removed. After each adaptive step the leave-one-out cross-validation coefficient $\left(Q^{2}\right)$ was calculated to evaluate the model's predictive power (Quicken et al. [23]). Once the meta-model was of sufficient quality (high 
$\left.R^{2} / Q^{2}\right)$, the main and total Sobol sensitivity indices could be derived analytically from the constructed meta-model.

The agPCE framework was executed multiple times with different settings for $\varepsilon_{1}, \varepsilon_{2}, Q_{\text {trgt }}^{2}, z_{\max }$, and $j_{\max }$ in order to find the meta-model with the highest possible $Q^{2}$. The thresholds $\varepsilon_{1}$ and $\varepsilon_{2}$ were varied as follows: $\varepsilon_{1}=[0.001 ; 0.05] *(1-$ $\left.Q_{\text {trgt }}^{2}\right)$ and $\varepsilon_{2}=[0.05 ; 2] * \varepsilon_{1}$, in which $Q_{\text {trgt }}^{2}$ was set to either 0.99 or 0.999 . In addition, the "low rank" truncation scheme was applied. This scheme only allowed polynomials with a maximum polynomial order, $z_{\max }$ and a maximum interaction order $j_{\max }$, to be included in the meta-model[43]. Based on $z_{\max }$ and $j_{\max }$ of 6 and 2 , respectively, we estimated that 363 simulations would be needed to fit the metamodels' coefficients. However, because the agPCE method is capable of removing non-significant polynomials, it is possible that higher order polynomials and/or interaction terms can be included instead. Therefore, we decided to vary $z_{\max }$ and $j_{\text {max }}$ between 1 and 6 , and 1 and 5 , respectively. Construction of a meta-model was terminated when either $Q_{\text {trgt }}^{2}$ was reached or when expansion coefficients could not be calculated due to an insufficient number of model evaluations. Using the above-mentioned settings, a large set of meta-models was constructed and the meta-model with the highest $Q^{2}$ was selected as final model. Meta-models were constructed for each WSS indices on the dataset of 363 CFD simulations that were performed prior to the sensitivity analysis (Figure 4.1).

\subsection{Results}

\subsubsection{Virtual patient database}

PWPM simulations for each realisation of the virtual database resulted in different pressure and flow waveforms within the $1 \mathrm{D}$ vascular domain. The $95^{\text {th }}$ percentile intervals of the waveforms obtained at locations corresponding with the aneurysm in- and outlets are depicted in Figure 4.4 and Figure 4.5, respectively. As can be seen from these figures, the virtual database allowed us to create a large variety of different waveforms with varying mean, peak and diastolic values. Compared to characteristic points in ICA waveforms defined by Ford et al. [44], our M0, H0, M2, and P3 peaks show similar timings and normalized flow (see Table 4.3). Although our normalized flow of $\mathrm{P} 1$ is correct, the timing of $\mathrm{P} 1$ occurs almost twice as late. In addition, our ICA waveforms do neither show an M1 nor a P2 characteristic peak. Finally, our mean ICA flow curve has a mean and peak value of 4.62 and $7.22 \mathrm{ml} \mathrm{s}^{-1}$. 


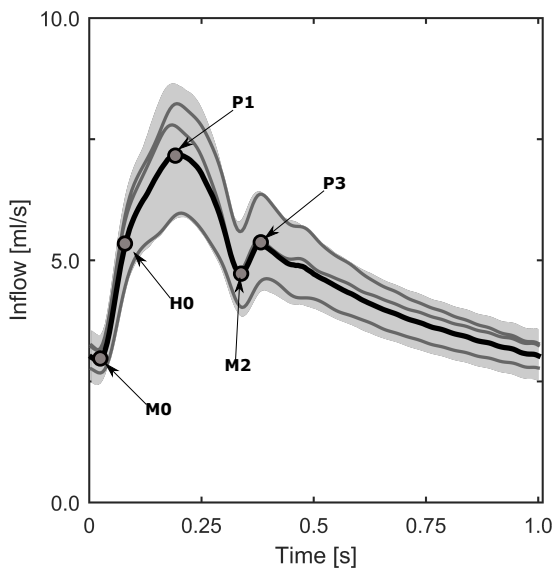

Figure 4.4: The different inflow signals obtained with the pulse wave propagation model for all realizations of the virtual database. The light gray area indicates the $95^{\text {th }}$ percentile interval of all realizations while the median waveform is presented in black. Three different realizations are shown in gray.

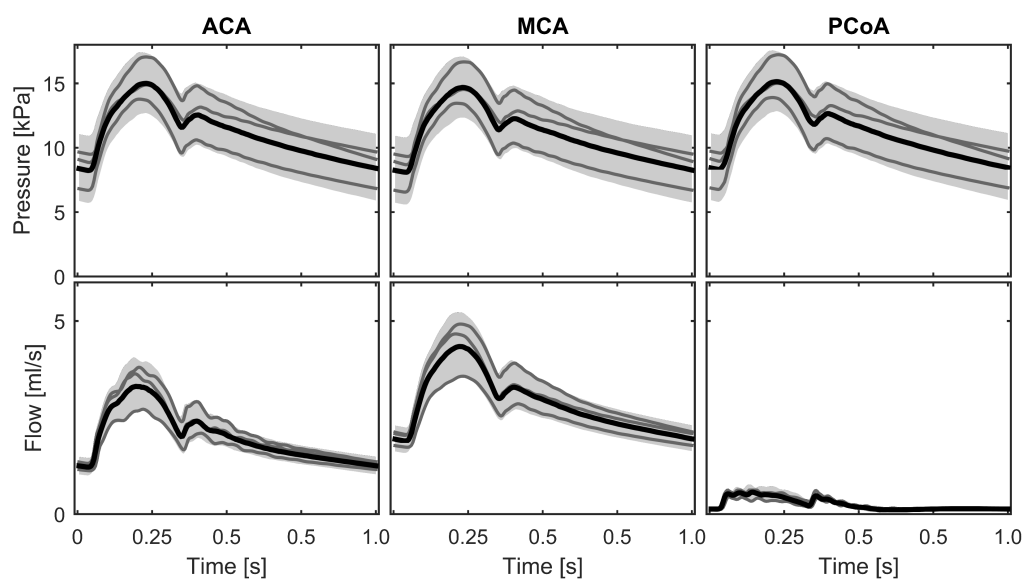

Figure 4.5: Pressure and flow waveforms of the aneurysm outlets obtained with the pulse wave propagation model for all realizations of the virtual database. The light gray area indicates the $95^{\text {th }}$ percentile interval of all realizations while the median waveform is presented in black. Three different realizations are shown in gray. ACA: anterior cerebral artery, MCA: middle cerebral artery, PCoA: posterior communicating artery. The mean flows of the median waveforms are $2.01,2.87$, and $0.26 \mathrm{ml} \mathrm{s}^{-1}$, for the right ACA, MCA, and PCoA, respectively. 
Table 4.3: Characteristic points of the ICA flow curves

\begin{tabular}{|c|c|c|c|c|}
\hline & \multicolumn{2}{|c|}{ Timing $[\mathrm{ms}]^{*}$} & \multicolumn{2}{|c|}{ Normalized Flow [-] } \\
\hline & \multicolumn{2}{|c|}{ Mean (SD) } & \multicolumn{2}{|c|}{ Mean (SD) } \\
\hline & PWPM & Ford et al. [44] & PWPM & Ford et al. [44] \\
\hline M0 & $-51(4.1)$ & $-61(7)$ & $0.64(0.05)$ & $0.68(0.05)$ \\
\hline $\mathrm{HO}$ & o (o) & $o(0)$ & $1.1(0.06)$ & $1.18(0.07)$ \\
\hline P1 & 116 (12) & $45(4)$ & 1.56 (o.09) & 1.66 (o.16) \\
\hline M1 & $\mathrm{X}$ & $141(20)$ & $\mathrm{X}$ & $1.20(0.09)$ \\
\hline P2 & $\mathrm{X}$ & $187(25)$ & $\mathrm{X}$ & $1.22(0.10)$ \\
\hline M2 & $249(37)$ & $280(21)$ & $1.03(0.14)$ & $0.94(0.08)$ \\
\hline P3 & $298(43)$ & $350(8)$ & $1.18(0.12)$ & $1.09(0.04)$ \\
\hline
\end{tabular}

* Timings are with respect to $\mathrm{H} 0$.

\subsubsection{D-CFD simulations}

The $95^{\text {th }}$ percentile intervals of the WSS indices are shown in Table 4.4. The corresponding coefficients of variation are $17 \%, 18 \%$, and $11 \%$ for $\tau_{\mathrm{avg}, \max }, \tau_{\mathrm{avg}, \max }^{*}$, and $i_{\mathrm{os}, \max }$, respectively. The $14 \%$ variability found in $c_{\mathrm{A}, \text { low }}$ is of the same order of magnitude.

Spatial distributions of the median value and the local variation (quantified by the $95^{\text {th }}$ percentile interval) of $\tau_{\text {avg, } \max }$ are shown in Figure $4.6 \mathrm{~A}$ and Figure $4.6 \mathrm{~B}$, respectively. Large median $\tau_{\mathrm{avg}, \max }$ is present close to the ostium at the right side of the aneurysm. Local variations in $\tau_{\mathrm{avg}, \max }$ are visible along the right side of the aneurysm, from the ostium up to the top of the aneurysm. Locally $\tau_{\mathrm{avg}, \max }$ can vary as much as $3.4 \mathrm{~Pa}$. Similarly, the distributions of median values and the local variation of the OSI are shown in Figure 4.6C and Figure 4.6D, respectively. Figure 4.6C shows an OSI pattern with large values located at the front and to some extent the right midsection of the aneurysm. These regions with high OSI values are located around the regions containing high $\tau_{\text {avg, } \max }$. In contrast, low values of OSI are found in regions with high $\tau_{\mathrm{avg}, \max }$. Local variations in OSI are present mostly at the front side of the aneurysm but some variation is also visible at the right and left midsection of the aneurysm (Figure 4.6D). Despite the fact that most of the variations are small, variations can be as high as 0.37 [-].

\subsubsection{Sensitivity analysis}

The results of the sensitivity analysis are summarized in Table 4.5. Meta-models with $Q^{2}>0.98$ could be constructed for all WSS indices except $i_{\mathrm{os}, \max }$. For this WSS index the meta-model had a $Q^{2}$ of 0.85 . Convergence checks of the sensitivity 
Table 4.4: Percentiles of the wall shear stress indices for all realizations of the virtual database

\begin{tabular}{lllll}
\hline & & \multicolumn{3}{c}{ Percentiles } \\
\cline { 3 - 5 } Output of interest & Unit & $2.50 \%$ & $\mathbf{5 0} \%$ & $97.5 \%$ \\
\hline$\tau_{\text {avg }, \text { max }}^{*}$ & $\mathrm{~Pa}$ & 7.07 & $\mathbf{8 . 5}$ & 10.1 \\
$\tau_{\text {avg }, \text { max }}^{*}$ & - & 15 & $\mathbf{1 6 . 8}$ & 19.2 \\
$i_{\text {os }, \text { max }}$ & - & 0.39 & $\mathbf{0 . 4 4}$ & 0.49 \\
$C_{\mathrm{A}, \text { low }}$ & $\%$ & 73 & $\mathbf{8 1}$ & 87 \\
\hline
\end{tabular}

indices showed convergence for all WSS indices except $i_{\text {os, } \max }$. In addition, all constructed meta-models had an $R^{2}$ larger than o.89. Table 4.5 also shows the main and total sensitivity indices for all WSS indices. Main sensitivity indices $(S)$ for mean aortic inflow $\bar{q}_{\text {ao }}$ were larger than 0.89 for all WSS indices except $i_{\text {os, } \max }(S=0.69)$. The age index $i_{\text {age }}$, shows a direct contribution of $5 \%(S=$ $0.05)$ to the variance in $\tau_{\text {avg, } \text { max }}^{*}$. In addition, $i_{\text {age }}$ attributed $7 \%$ to the total output variance present within $i_{\mathrm{os}, \max }(S=0.07)$. The main sensitivity indices for the other systemic properties were all smaller than $5 \%$.

The differences between total and main sensitivity indices showed that all systemic properties were involved in interactions with other parameters $\left(S_{\mathrm{T}}-S>\right.$ 0 ). If a parameter is involved in interaction, it means that its effect on the WSS indices is depending on the value of another parameter. When these interactions were also considered the relevance of some systemic properties changed quite a bit. For example, $\bar{q}_{\mathrm{ao}}$ and $i_{\text {age }}$ were involved in interactions that contributed to $82 \%\left(S_{\mathrm{T}}=0.82\right)$ and $14 \%\left(S_{\mathrm{T}}=0.14\right)$ of the output variance present within $i_{\mathrm{os}, \max }$, respectively, whereas on their own they only contributed to $69 \%$ and $7 \%$, respectively. Nevertheless, even when interactions were taken into account, the mean aortic inflow $\bar{q}_{\text {ao }}$ remained by far the parameter with the largest contribution to the variance present within the WSS indices.

\subsection{Discussion}

In this study, we aimed to quantify the effect of variations in systemic properties on wall shear stress indices derived from 3D-CFD simulations of a cerebral aneurysm. Moreover, we assessed which of the systemic properties had the largest effect on the variability of the calculated wall shear stress-based risk indices. 
Time-averaged Wall Shear Stress
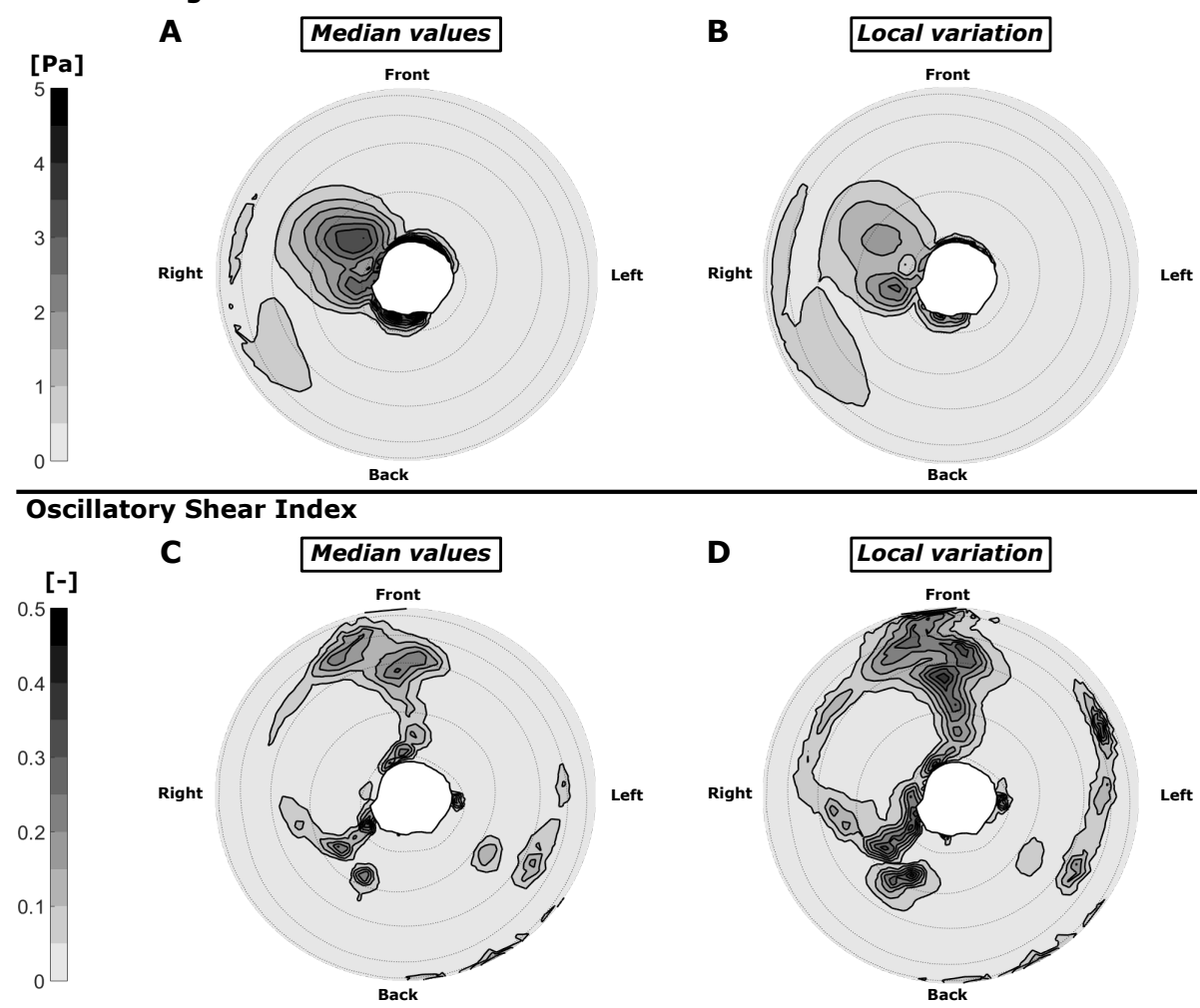

Figure 4.6: Lambert azimuthal equal area-plots of spatial distributions. Panel A and $C$ show the spatial distributions for the median values of respectively, the maximum time-averaged wall shear stress magnitude $\tau_{\mathrm{avg}, \max }$ and the maximum oscillatory shear index $i_{\mathrm{os}, \max }$. Local variations of both parameters are shown in Panel B and D. These variations are quantified by the range of the $95^{\text {th }}$ percentile interval.

\subsubsection{Major Findings}

Variations within the physiological ranges of mean arterial pressure, mean aortic inflow, total arterial compliance, mean cerebral blood flow,, and ageing-induced increase in radii and Young's moduli were applied to the PWPM. These variations resulted in variabilities in the WSS indices ranging between $11 \%$ and $18 \%$ (coefficients of variations). The $14 \%$ variability in percentage area with low time-averaged wall shear stress magnitude, $c_{\mathrm{A}, l o w}$, found in our study is of the same order of magnitude as the difference between ruptured and unruptured aneurysms as found 
Table 4.5: The Main and Total Sobol sensitivity indices $S$ and $S_{\mathrm{T}}$ for all wall shear stress indices (rows) for each of the five systemic properties (columns). In addition, the $R^{2}$ and $Q^{2}$ of the meta-models are shown on the right side of the table.

\begin{tabular}{|c|c|c|c|c|c|c|c|c|}
\hline & & \multicolumn{5}{|c|}{$\begin{array}{l}\text { Systemic properties } \\
\text { (input parameters) }\end{array}$} & \multicolumn{2}{|c|}{$\begin{array}{l}\text { Quality of the } \\
\text { regression model }\end{array}$} \\
\hline & & $\bar{p}_{\mathrm{a}}$ & $\bar{q}_{\mathrm{ao}}$ & $C_{\mathrm{T}}$ & $\bar{q}_{\mathrm{c}}$ & $i_{\text {age }}$ & $R^{2}$ & $Q^{2}$ \\
\hline \multirow[b]{2}{*}{$\tau_{\text {avg }, \max }$} & $S$ & 0.0086 & 0.9515 & 0.0004 & 0.0161 & 0.0208 & 0.998 & 0.997 \\
\hline & $S_{\mathrm{T}}$ & 0.0096 & 0.9535 & 0.0009 & 0.0164 & 0.0228 & & \\
\hline \multirow{2}{*}{$\tau_{\mathrm{avg}, \max }^{*}$} & $S$ & 0.0067 & 0.8929 & 0.015 & 0.0153 & 0.0513 & 0.985 & 0.981 \\
\hline & $S_{\mathrm{T}}$ & 0.0106 & 0.9086 & 0.0193 & 0.0202 & 0.0626 & & \\
\hline \multirow[b]{2}{*}{$i_{\mathrm{os}, \max }$} & $S$ & 0.0355 & 0.6931 & 0.0117 & 0.0251 & 0.0753 & 0.897 & 0.849 \\
\hline & $S_{\mathrm{T}}$ & 0.0876 & 0.8232 & 0.0697 & 0.0747 & 0.1401 & & \\
\hline \multirow{2}{*}{$C_{\mathrm{A}, l o w}$} & $S$ & 0.0055 & 0.9414 & 0.0031 & 0.0139 & 0.0197 & 0.987 & 0.983 \\
\hline & $S_{\mathrm{T}}$ & 0.0104 & 0.9552 & 0.0115 & 0.0171 & 0.0257 & & \\
\hline
\end{tabular}

Sensitivity indices larger than $5 \%$ are shown in bold.

by Jou et al. [45] and Zhang et al. [46], $16 \%$ and $13.6 \%$, respectively. However, the difference of $\mathbf{2 8} \%$, as found by, Xiang et al. [12], is much larger. Moreover, a study of Qiu et al. [47] found a difference of $4.9 \%$ and $25 \%$ for narrow-neck and wide-neck aneurysms, respectively. The variability in $c_{\mathrm{A}, l o w}$ due to variations in systemic properties found in this study is at least equal to a large portion of the variability between ruptured and unruptured aneurysms. Therefore, it might be possible that the uncertainties within this WSS index can lead to erroneous rupture predictions when this WSS index is used in multidisciplinary rupture prediction models. The effect of variations in systemic properties on this WSS index should therefore be taken into account.

Compared to other studies[15, 48-50], our variabilities in WSS indices are mostly within the same order of magnitude, but our experimental design was significantly different. First of all, previous studies only changed inflow BCs, whereas we induced changes in both in- and outflow BCs. We induced these changes by varying physiological systemic properties instead of changing the waveforms directly. This allowed us not only to assess the effect of variations in waveforms on the variability in the WSS indices, but also to identify physiological systemic properties responsible for this effect. Moreover, we did apply a global sensitivity analysis and not a local one. This made it possible to assess not only the direct effects, but also the interaction effects between multiple systemic properties. The interaction effects for the systemic properties indicate that the effects of variations in these parameters on the WSS indices, are depending on the values of other systemic properties. So, in order to assess the "full" influence of certain parameters on the WSS indices, it is 
necessary to take into account the variability of other input parameters at the same time. In order to do so, global sensitivity analysis is indispensable.

Although at first thought one might expect the amount of flow towards the aneurysm to be the most important factor for aneurysm rupture, our sensitivity analysis showed that mean aortic inflow $\bar{q}_{\text {ao }}$ and not mean cerebral flow $\bar{q}_{\mathrm{c}}$ was the systemic property most accountable for the variability in our WSS indices. However, it is important to note that an increase in $\bar{q}_{\text {ao }}$ does not simply increase blood flow throughout the whole arterial 1D domain. To elucidate this, we will further elaborate on the way we implemented mean aortic inflow $\bar{q}_{\text {ao }}$ and mean cerebral flow $\bar{q}_{\mathrm{c}}$ in the PWPM. To assess $R_{\mathrm{p}}$, mean arterial pressure $\bar{p}_{\text {ao }}$ was used. When $\bar{p}_{\text {ao }}$ is not changed, an increase in $\bar{q}_{\text {ao }}$ will result in a decrease of total peripheral resistance. Given $\bar{p}_{\text {ao }}$ and the pre-set amount of cerebral blood $\bar{q}_{\mathrm{c}}$, the peripheral resistance of the brain compartment does not change as $R_{\mathrm{p}, \text { brain }}=\bar{p}_{\mathrm{ao}} / \bar{q}_{\mathrm{c}}$. As a consequence, the extra inflow will be directed towards the body. Besides the increased body inflow, the altered body windkessel resistances result in different reflections which transform pressure and flow waveforms. These altered waveforms travel towards the brain and result in different pressure and flow waveforms in the aneurysm in- and outlets. Because these waveforms are used to fit the windkessel parameters, different BCs are obtained, which in turn lead to different values for the WSS indices.

In contrast, increasing $\bar{q}_{\mathrm{c}}$ does lead to a reduced cerebral peripheral resistance, once again, assuming constant mean blood pressure. As a consequence, the adjusted cerebral resistances yield different pressure and flow reflections. Because of the increased inflow and the altered cerebral windkessel resistances, different pressure and flow waveforms arise in the aneurysm in- and outlet vessels leading to different WSS indices. Despite the fact that changing $\bar{q}_{\mathrm{c}}$ and $\bar{q}_{\text {ao }}$ both influence pressure and flow waveforms, our sensitivity analysis showed that the WSS indices are much more affected by variations in waveforms caused by changes in systemic body properties $\left(\bar{q}_{\text {ao }}\right)$ than by variations caused by changes in systemic brain properties $\left(\bar{q}_{\mathrm{c}}\right)$.

Our CFD simulation-derived WSS and OSI patterns are comparable to previous studies[16, 51, 52]. Our spatial distributions showed high median WSS close to the ostium on the right side of the aneurysm. This WSS distribution is caused by a flow jet entering the aneurysm. Because of the orientation of the aneurysm with respect to the ICA, this jet hits the aneurysm wall on the right side. This region of high WSS corresponds roughly with the bleb visible on the right side of the aneurysm (Figure 4.3). Previous studies also showed that regions exposing these blebs were related to regions of elevated WSS[51-53]. Sometimes the development of these blebs was even initiated by the flow jet $[51,52]$. The local variations in WSS, visible in the region of the jet's impact location, indicate that the impact location of the jet differs slightly between simulations. 
High OSI values were found around regions with high time-averaged WSS (around the impact location of the jet) and can be explained by dissipation of the jet. The high OSI along the front side of the aneurysm arises because blood flow leaves the aneurysm along the front wall of the aneurysm. Large local OSI variations indicate that the way the jet dissipates, and the way blood leaves the aneurysm, is highly influenced by variations in in- and outlet waveforms caused by variations in systemic properties.

\subsubsection{Limitations and Future work}

Assessing proper boundary conditions for 3D aneurysm models by using a pulse wave propagation model has been applied in several studies[16, 17]. We believe that combining a well-established PWPM with physiological parameter ranges gives a good indication of the possible variation in WSS indices

In analogy with Mulder et al. [54], we compared our ICA flows with ICA flow characteristics defined by Ford et al. [44]. In general, our ICA characteristics show great similarities with the ones presented by Ford et al. [44] All characteristics, except the timing of the first systolic peak P1, show similar values. Our P1 peak appears almost twice as late. In addition, similarly as Mulder et al. [54] we observed no P2 and M1 peak. These similar findings with Mulder et al. [54]. are most likely caused because we use the same 1D geometry as Mulder et al. [54]. The "late timing" of our $\mathrm{P} 1$ peak, in combination with the fact that our flow waveform looks rather similar to our ICA pressures, indicates that our distal vasculature might be to "stiff". However, the true reason behind this large timing of the $\mathrm{P} 1$ peak is something that has to be investigated in future research.

Although we varied our input parameters within their physiological ranges, we have to keep in mind that there are more sources of uncertainty that can contribute to the uncertainty within WSS indices. For example, other parameters of the PWPM, besides the one varied here, could alter the BCs as well. In addition, there are factors/mechanisms that are not yet included in the PWPM. For example, the model does not contain regulatory mechanisms, such as cerebral autoregulation or the baroreflex. Such mechanisms regulate flow distributions and pressures by changing vessel diameter, peripheral resistances, heartrate, and stroke volume. In turn, the shape of pressure and flow waveforms will most likely change. As a result, different BCs for the CFD simulations are obtained which might change the WSS indices. Although, we did not include such mechanisms in this study, they can be added to the PWPM. 
Furthermore, we did only analyze one aneurysm geometry and we did not include the variability due to measurement and segmentation errors of the aneurysm geometry. Although adding a proper uncertainty to the parameterization of 3D geometries is challenging[55], previous research has shown that segmentation of the parent artery and the aneurysm can influence the flow fields obtained with CFD [56-58]. In addition, even imaging with different modalities can lead to changes in mean WSS of up to $34 \%$ [59]. For the above reasons, these uncertainties should be included in future work. Taking into account sources of uncertainty, such as the ones described above, the variability within the WSS indices would most likely become larger. However, our approach could give us insight in which of these sources of uncertainty have an important effect on the variability within the WSS indices.

In contrast to other studies[16, 6o], we did not normalize the WSS with respect to the aneurysm inflow or WSS in the parent artery. However, our aim was to assess the effect of different BCs on the WSS indices, and scaling the WSS indices would only cover the influence of the systemic properties.

We used a global variance-based sensitivity method, which are considered the best available practice for sensitivity analysis[61]. Although only the meta-model of $\tau_{\mathrm{avg}, \max }$ reached the pre-set $Q_{\mathrm{trgt}}^{2}$, the sensitivity indices of all WSS indices, except $i_{\mathrm{os} \text {, max }}$ showed convergence. Although it is possible that the contributions of the different systemic properties may change slightly if we would construct new meta-models using more model outputs, we do not expect this to change our final conclusions because of the very dominant influence of the parameter $\bar{q}_{\text {ao }}$.

\subsection{Conclusion}

We created a methodology that allows quantification of variabilities in aneurysm wall shear stress indices that result from variations in systemic properties. Estimated variabilities in WSS indices were of the same order of magnitude as differences in these WSS indices between ruptured and unruptured aneurysms. Because variations in systemic properties influence both in- and outlet BCs at the same time, we conclude that patient-specific systemic properties and their effect on in- and outlet BCs, should be taken into account when using WSS indices in multidisciplinary aneurysm rupture models. Hereto, uncertainty and sensitivity quantification is indispensable. 


\section{References}

1. Greving, J. P., Wermer, M. J. H., Jr, R. D. B. et al. Development of the PHASES score for prediction of risk of rupture of intracranial aneurysms : a pooled analysis of six. Lancet neurology 13, 59-66 (2014).

2. Detmer, F. J., Chung, B. J., Jimenez, C., Hamzei-sichani, F. \& Kallmes, D. Associations of hemodynamics , morphology, and patient characteristics with aneurysm rupture stratified by aneurysm location. Neuroradiology Journal 61, 275-284 (2019).

3. Jing, L., Fan, J., Wang, Y., Li, H., Wang, S. \& Yang, X. Morphologic and Hemodynamic Analysis in the Patients with Multiple Intracranial Aneurysms : Ruptured versus Unruptured. PLoS ONE, 1-12 (2015).

4. Xiang, J., Natarajan, S. K., Tremmel, M. et al. Hemodynamic-Morphologic Discriminants for Intracranial Aneurysm Rupture. Stroke 42, 144-152 (2011).

5. Kleinloog, R., Mul, N. D., Post, J. A. \& Rinkel, G. J. E. Risk Factors for Intracranial Aneurysm Rupture : A systematic review. Neurosurgery 82, 431-440 (2018).

6. Liang, L., Steinman, D. A., Brina, O., Chnafa, C., Cancelliere, N. M. \& Pereira, V. M. Towards the Clinical utility of CFD for assessment of intracranial aneurysm rupture - a systematic review and novel parameter-ranking tool. Journal of NeuroInterventional Surgery 11, 153-158 (2019).

7. Xiang, J., Natarajan, S. K., Tremmel, M. et al. Hemodynamic-Morphologic Discriminants for Intracranial Aneurysm Rupture. Stroke 42, 144-152 (2011).

8. Miura, Y., Ishida, F., Umeda, Y. et al. Low Wall Shear Stress Is Independently Associated With the Rupture Status of Middle Cerebral Artery Aneurysms. Stroke 44, 519-521 (2013).

9. Connolly, E. S., Rabinstein, A. A., Carhuapoma, J. R. et al. AHA / ASA Guideline Guidelines for the Management of Aneurysmal Subarachnoid Hemorrhage A Guideline for Healthcare Professionals From the American Heart Association / American Stroke Association. Stroke 43 (2012).

10. Detmer, F. J., Chung, B. J., Mut, F. et al. Development of a statistical model for discrimination of rupture status in posterior communicating artery aneurysms. Acta neurochirurgica (2018).

11. Bisbal, J. \& Engelbrecht, G. Prediction of Cerebral Aneurysm Rupture Using Hemodynamic , Morphologic and Clinical Features : A Data Mining Approach Prediction of Cerebral Aneurysm Rupture using Hemodynamic, Morphologic and Clinical Features : A Data Mining Approach. proc.Int.Conf.Database Expert Syst. Appl. (2011).

12. Xiang, J., Yu, J., Snyder, K. V., Levy, E. I., Siddiqui, A. H. \& Meng, H. Hemodynamic - morphological discriminant models for intracranial aneurysm rupture remain stable with increasing sample size. Journal of NeuroInterventional Surgery 8, 104-110 (2016).

13. Xiang, J., Yu, J., Choi, H. et al. Rupture Resemblance Score ( RRS ): toward risk strati fi cation of unruptured intracranial aneurysms using hemodynamic - morphological discriminants. journal of neuroin o, 1-6 (2014).

14. Detmer, F. J., Jae, B., Fernando, C. et al. Development and internal validation of an aneurysm rupture probability model based on patient characteristics and aneurysm location , morphology , and hemodynamics. International Journal of Computer Assisted Radiology and Surgery 13, 17671779 (2018).

15. Sarrami-Foroushani, A., Lassila, T., Gooya, A., Geers, A. J. \& Frangi, A. F. Uncertainty quantification of wall shear stress in intracranial aneurysms using a data-driven statistical model of systemic blood flow variability. Journal of Biomechanics 49, 3815-3823 (2016). 
16. Marzo, A., Singh, P., Larrabide, I. et al. Computational hemodynamics in cerebral aneurysms: The effects of modeled versus measured boundary conditions. Annals of Biomedical Engineering 39, 884-896 (2011).

17. Mulder, G., Marzo, A., Bogaerds, A. C. B. et al. Patient-Specific Modeling of Cerebral Blood Flow: Geometrical Variations in a 1D Model. Cardiovascular Engineering and Technology 2, 334-348 (2011).

18. Reymond, P., Merenda, F., Perren, F. et al. Validation of a one-dimensional model of the systemic arterial tree. American journal of physiology. Heart and circulatory physiology 297, H208-22 (2009).

19. Reymond, P., Bohraus, Y., Perren, F., Lazeyras, F. \& Stergiopulos, N. Validation of a patient-specific one-dimensional model of the systemic arterial tree. Blood, 1173-1183 (2011).

2o. Alastruey, J., Parker, K. H., Peiró, J., Byrd, S. M. \& Sherwin, S. J. Modelling the circle of Willis to assess the effects of anatomical variations and occlusions on cerebral flows. Journal of Biomechanics 40, 1794-1805 (2007).

21. Sarrami-Foroushani, A., Lassila, T. \& Frangi, A. F. Virtual endovascular treatment of intracranial aneurysms: models and uncertainty. Wiley Interdisciplinary Reviews: Systems Biology and Medicine, e1385 (2017).

22. Willemet, M., Chowienczyk, P. \& Alastruey, J. A database of virtual healthy subjects to assess the accuracy of foot-to-foot pulse wave velocities for estimation of aortic stiffness. American Journal of Physiology - Heart and Circulatory Physiology 309, H663-H675 (2015).

23. Quicken, S., Donders, W. P., van Disseldorp, E. M. J. et al. Application of an Adaptive Polynomial Chaos Expansion on Computationally Expensive Three-Dimensional Cardiovascular Models for Uncertainty Quantification and Sensitivity Analysis. Journal of Biomechanical Engineering 138, 121010 (2016).

24. O’Rourke, M. F. Arterial aging: Pathophysiological principles. Vascular Medicine 12, 329-341 (2007).

25. Sobol', I. M. On the distribution of points in a cube and the approximate evaluation of integrals. USSR Computational Mathematics and Mathematical Physics 7, 86-112 (1967).

26. Ahlgren, Å. R., Hansen, F., Sonesson, B. \& Länne, T. Stiffness and diameter of the common carotid artery and abdominal aorta in women. Ultrasound in Medicine and Biology 23, 983-988 (1997).

27. Van der Heijden-Spek, J. J., Staessen, J. a., Fagard, R. H., Hoeks, a. P., Boudier, H. a. \& van Bortel, L. M. Effect of age on brachial artery wall properties differs from the aorta and is gender dependent: a population study. Hypertension 35, 637-642 (2000).

28. Millasseau, S. C., Stewart, A. D., Patel, S. J., Redwood, S. R. \& Chowienczyk, P. J. Evaluation of Carotid - Femoral Pulse Wave Velocity Influence of Timing Algorithm and Heart Rate, 222-226 (2005).

29. Elstad, M. \& WallØe, L. Heart rate variability and stroke volume variability to detect central hypovolemia during spontaneous breathing and supported ventilation in young, healthy volunteers. Physiological Measurement 36, 671-681 (2015).

30. Holme, N. L. A., Rein, E. B. \& Elstad, M. Cardiac stroke volume variability measured non-invasively by three methods for detection of central hypovolemia in healthy humans. European Journal of Applied Physiology 116, 2187-2196 (2016).

31. Laskey, W. K., Parker, H. G., Ferrari, V. A., Kussmaul, W. G. \& Noordergraaf, A. Estimation of total systemic arterial compliance in humans. Journal of applied physiology (Bethesda, Md. : 1985) 69, 112-9 (1990). 
32. Henriksen, O. M., Larsson, H. B. W., Hansen, A. E., Law, I., Rostrup, E. \& Gru, J. M. Estimation of Intersubject Variability of Cerebral Blood Flow Measurements Using MRI and Positron Emission Tomography. 1299, 1290-1299 (2012).

33. Sonesson, B., Länne, T., Hansen, F. \& Sandgren, T. Infrarenal aortic diameter in the healthy person. European Journal of Vascular Surgery 8, 89-95 (1994).

34. Thijssen, D. H., Carter, S. E. \& Green, D. J. Arterial structure and function in vascular ageing: Are you as old as your arteries? Journal of Physiology 594, 2275-2284 (2016).

35. Kroon, W., Huberts, W., Bosboom, M. \& van de Vosse, F. A numerical method of reduced complexity for simulating vascular hemodynamics using coupled oD lumped and $1 \mathrm{D}$ wave propagation models. Computational and Mathematical Methods in Medicine 2012, 1-10 (2012).

36. Ahlborg, G. \& Jensen-Urstad, M. Arm blood flow at rest and during arm exercise. Journal of Applied Physiology 70, 928-933 (2017).

37. Hill, E., Housh, T., Smith, C., Keller, J., Schmidt, R. \& Johnson, G. The contributions of arterial cross-sectional area and time-averaged flow velocity to arterial blood flow. Journal of Medical Ultrasound 26, 186 (2018).

38. Olufsen, M. S. \& Peskin, C. Numerical Simulation and Experimental Validation of Blood Flow in Arteries with Structured-Tree Outflow Conditions. 28, 1281-1299 (2000).

39. Hulsen, M. TFEM A toolkit for the finite element method User's Guide (2009).

40. Codina, R., Oñate, E. \& Cervera, M. The intrinsic time for the streamline upwind/Petrov-Galerkin formulation using quadratic elements. Computer Methods in Applied Mechanics and Engineering 94, 239-262 (1992).

41. Saltelli, A., Annoni, P., Azzini, I., Campolongo, F., Ratto, M. \& Tarantola, S. Variance based sensitivity analysis of model output. Design and estimator for the total sensitivity index. Computer Physics Communications 181, 259-270 (2010).

42. Donders, W. P., Huberts, W., van de Vosse, F. N., Delhaas, T., de Vosse, v. F. \& Donders, W. P. Personalization of models with many model parameters: an efficient sensitivity analysis approach. International Journal for Numerical Methods in Biomedical Engineering 31, n/a-n/a (2015).

43. Blatman, G. \& Sudret, B. Adaptive sparse polynomial chaos expansion based on least angle regression. Journal of Computational Physics 230, 2345-2367 (2011).

44. Ford, M. D., Alperin, N., Lee, S. H., Holdsworth, D. W. \& Steinman, D. A. Characterization of volumetric flow rate waveforms in the normal internal carotid and vertebral arteries. Physiological Measurement 26, 477-488 (2005).

45. Jou, L.-D., Lee, D. H., Morsi, H. \& Mawad, M. E. Wall Shear Stress on Ruptured and Unruptured Intracranial Aneurysms at the Internal Carotid Artery. American Journal of Neuroradiology 29, 1761-1767 (2008).

46. Zhang, Y., Tian, Z., Jing, L., Zhang, Y., Liu, J. \& Yang, X. Bifurcation type and larger low shear area are associated with rupture status of very small intracranial aneurysms. Frontiers in Neurology 7 , 1-6 (2016).

47. Qiu, T., Jin, G., Xing, H. \& Lu, H. Association between hemodynamics, morphology, and rupture risk of intracranial aneurysms: a computational fluid modeling study. Neurological Sciences 38, 1009-1018 (2017).

48. Jansen, I. G. H., Schneiders, J. J., Potters, W. V. et al. Generalized versus Patient-Specific Inflow Boundary Conditions in Computational Fluid Dynamics Simulations of Cerebral Aneurysmal Hemodynamics. Americal Journal of NeuroRadiology 35, 1543-1548 (2014). 
49. Karmonik, C., Yen, C., Diaz, O., Klucznik, R., Grossman, R. G. \& Benndorf, G. Temporal variations of wall shear stress parameters in intracranial aneurysms: importance of patient-specific inflow waveforms for CFD calculations. Acta neurochirurgica 152, 1391-1398 (2010).

5o. Karmonik, C., Yen, C., Grossman, R. G., Klucznik, R. \& Benndorf, G. Intra-aneurysmal flow patterns and wall shear stresses calculated with computational flow dynamics in an anterior communicating artery aneurysm depend on knowledge of patient-specific inflow rates. Acta Neurochirurgica 151, 479-485 (2009).

51. Cebral, J. R., Sheridan, M. \& Putman, C. M. Hemodynamics Before and After Bleb Formation in Cerebral Aneurysms. American Journal of Neuroradiology 31, 304-310 (2010).

52. Cebral, J. R., Castro, M. A., Burgess, J. E., Pergolizzi, R. S., Sheridan, M. J. \& Putman, C. M. Characterization of cerebral aneurysms for assessing risk of rupture by using patient-specific computational hemodynamics models. AJNR. American journal of neuroradiology 26, 2550-9 (2008).

53. Tateshima, S., Murayama, Y., Villablanca, J. P. et al. In vitro measurement of fluid-induced wall shear stress in unruptured cerebral aneurysms harboring blebs. Stroke 34, 187-192 (2003).

54. Mulder, G., Bogaerds, A. C. B., Rongen, P. \& van de Vosse, F. N. The influence of contrast agent injection on physiological flow in the circle of Willis. Medical Engineering and Physics 33, 195-203 (2011).

55. Sankaran, S., Grady, L. \& Taylor, C. A. Impact of geometric uncertainty on hemodynamic simulations using machine learning. Computer Methods in Applied Mechanics and Engineering 297, 167-190 (2015).

56. Cebral, J. R., Castro, M. a., Appanaboyina, S., Putman, C. M., Millan, D. \& Frangi, A. F. Efficient pipeline for image-based patient-specific analysis of cerebral aneurysm hemodynamics: Technique and sensitivity. IEEE Transactions on Medical Imaging 24, 457-467 (2005).

57. Castro, M. A., Putman, C. M. \& Cebral, J. R. Computational fluid dynamics modeling of intracranial aneurysms: Effects of parent artery segmentation on intra-aneurysmal hemodynamics. American Journal of Neuroradiology 27, 1703-1709 (2006).

58. Voß, S., Beuing, O., Janiga, G. \& Berg, P. Multiple Aneurysms AnaTomy CHallenge 2018 (MATCH)—Phase Ib: Effect of morphology on hemodynamics. Plos One 14, e0216813 (2019).

59. Geers, A. J., Larrabide, I., Radaelli, A. G. et al. Patient-specific computational hemodynamics of intracranial aneurysms from $3 \mathrm{D}$ rotational angiography and CT angiography: An in vivo reproducibility study. American Journal of Neuroradiology 32, 581-586 (2011).

6o. Geers, A. J., Morales, H. G., Larrabide, I., Butakoff, C., Bijlenga, P. \& Frangi, A. F. Wall shear stress at the initiation site of cerebral aneurysms. Biomechanics and Modeling in Mechanobiology 16, 97-115 (2017).

61. Huberts, W., Donders, W. P., Delhaas, T. \& van de Vosse, F. N. Applicability of the polynomial chaos expansion method for personalization of a cardiovascular pulse wave propagation model. International Journal for Numerical Methods in Biomedical Engineering 30, 1679-1704 (2014). 



\section{A new OD Junction Element for Pressure Losses at Bifurcations}

Based on: van den Boom, T., Stevens, R.R.F., Delhaas, T., van de Vosse, F.N. \& Huberts, W. 0D lumped approach to incorporate the dynamic part of the pressure at vessel junctions in a 1D wave propagation model International Journal for Numerical Methods in Biomedical Engineering, 34(9). 


\section{Abstract}

A benchmark study by Boileau et al. [1]. tested six commonly used numerical schemes for 1D wave propagation, for their ability to capture the main features of pressure, flow, and area waveforms in large arteries. While all numerical schemes showed good agreement in pressure and flow waveforms for smaller arterial networks, the Simplified Trapezium-rule Method (STM) proposed by Kroon et al. [2] showed an overestimation for the systolic pressure of $1 \%$ in proximal regions and an underestimation of $3 \%$ in distal regions in comparison with the five other schemes when using a larger arterial network, published as the ADAN 56 model. The authors attributed this difference to the neglection of the dynamic part of the pressure at vessel junctions. Carson et al. [3] resolved these differences by proposing two methods to implement the dynamic part of the pressure in the STM scheme. In the present study, an alternative method is introduced extending the work by Kroon et al. [2] This alternative method consists of a new $0 \mathrm{D}$ element which is placed at vessel junctions. The strength of this new element is the ease of implementation and its flexible coupling with other elements, without introducing additional degrees of freedom or the need of a penalty function. This new approach is compared to five other numerical schemes which already have the dynamic part of the pressure incorporated. The new method shows excellent agreement with these schemes for the ADAN 56 model. 


\subsection{Introduction}

N E dimensional (1D) network models have extensively been used to investigate blood pressure and blood flow wave propagation phenomena in arteries and veins[4-6]. These so-called pulse wave propagation models allow the assessment of the effects of vascular disease on the pressure and flow waveforms, wave reflections, and the relation between central and peripheral pressures. The ease of use and the relatively low costs make 1D models also attractive for vascular intervention planning[7] or for estimating boundary conditions for higher dimensional models[8].

These 1D network models consist of connected elements that locally describe the relation between pressure, area, and flow. The pressure and flow relations are based on the 1D mass and momentum equations[9]. At terminal branches, the 1D network is often truncated with, for example, $0 \mathrm{D}$ windkessel elements [7] or structured outflow trees[10]. To solve the governing equations derived from $0 \mathrm{D}$ and 1D models, different mathematical formulations and corresponding numerical methods have been proposed. These methods vary from each other by using different formulations of the $1 \mathrm{D}$ equations and different constitutive laws to relate area and pressure. In addition, they differ with respect to the velocity profiles used to estimate the friction and convective terms, choice in boundary conditions, the way of coupling at junctions, and the numerical schemes[11].

Recently, Boileau et al. [1] compared six numerical schemes that are commonly used for 1D arterial blood flow modeling to investigate the effect of different numerical implementations on the pressure and flow waveforms in several benchmark problems while using the same velocity profile, boundary conditions and constitutive laws[1]. The numerical schemes considered were: Locally Conservative Galerkin[12] (LCG), Discontinuous Galerkin[13] (DCG), Galerkin Least-Squares Finite Element Method[14] (FEM), Finite Volume Method[15] (FVM), MacCormack Finite Difference Method[16] (McC) and the Simplified Trapezoidal Rule Method[2] (STM). The results showed good agreement among all numerical schemes for six benchmark problems (i.e.: a reflection free tube, a tube mimicking the common carotid artery, the upper thoraric aorta, an aortic bifurcation, and experimental arterial networks with 37 and 56 of the larger arteries, published as the ADAN 56 model). It was concluded that all schemes are able to solve nonlinear 1D equations and to capture the main features of pulse wave propagation. However, for the ADAN 56 model, noticeable discrepancies were obtained when using the STM in comparison to the other numerical schemes. While the STM showed to be able to solve the system of equations within minutes [1, 2], systolic pressure was overestimated at proximal locations by $1 \%$ (i.e. $2 \mathrm{mmHg}$ ) and underestimated by $3 \%$ at distal locations (i.e. $4 \mathrm{mmHg}$ ). Boileau et al. [1] hypothesized that these differences were caused by the 
anatomical configuration of the ADAN 56 model, which makes the model more sensitive to the coupling strategy at junctions. They also hypothesized that the discrepancies found in the STM model, were the result of neglecting the dynamic part of the pressure $\left(\frac{1}{2} \rho u^{2}\right)$ at junctions[1]. Carson et al. [3] demonstrated that adding the dynamic pressure at junctions to the STM model indeed eliminated the differences found by Boileau et al. [1].

Carson et al. [3] proposed two methods to constrain the conservation of dynamic pressure at vessel junctions: Lagrange multipliers and a penalty method. Despite the fact that these correction methods showed good results, there are some downsides to these methods. Because the Lagrange multiplier method introduces additional degrees of freedom to the solution vector, the matrix needs to be expanded, which requires a more extensive storage[17]. For the penalty method, on the other hand, it is difficult to choose the required penalty parameter, the so-called penalty number [3]. A high penalty number is needed to enforce the dynamic pressure constraints, however, if the penalty number is too high, the global matrix becomes ill-conditioned with respect to inversion[17].

The aim of this study is to develop a new element that, on the one hand, is in line with the approach by Kroon et al. [2] and on the other hand, straightforwardly implements the dynamic part of the pressure in the STM model. The approach is based on the fact that $0 \mathrm{D}$ and $1 \mathrm{D}$ elements are cast in the same form. This results in a simplified numerical scheme, in which pressure is the only state variable. After the pressures are calculated on nodal level, the flow can be calculated on element level. This approach allows for flexible coupling between $0 \mathrm{D}$ and $1 \mathrm{D}$ elements, without the need for additional degrees of freedom or penalty functions. To demonstrate our simplified numerical scheme we benchmarked our implementations with the ADAN 56 geometry presented by Boileau et al. [1].

\subsection{Materials and Methods}

\subsubsection{D network model}

In this section, first, we describe the $1 \mathrm{D}$ computational domain (ADAN56) that consists of connected 1D arterial elements and is closed with three-element windkessel elements at truncating parts. At vascular junctions, a new $0 \mathrm{D}$ element is introduced (Figure 5.1). Secondly, we present the assembly of the equations for all different elements. In addition, the numerical method used to solve the scheme is explained. Finally, the simulations performed and their analysis are described. 


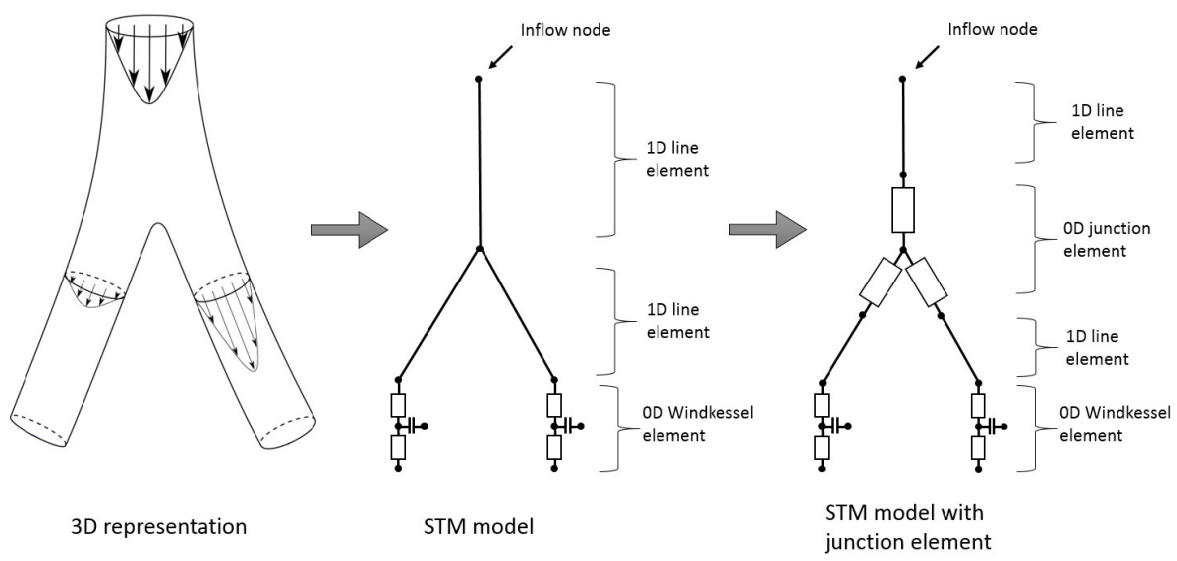

Figure 5.1: The implementation of the $0 D$ junction element in a simple bifurcation geometry.

1D computational domain

Because Boileau et al. [1], showed only discrepancies for the STM method in the ADAN 56 geometry, we chose to use the ADAN 56 model as well. The ADAN 56 model contains 56 large vessels of the human arterial system. It originates at the aortic arch and is truncated at the internal carotid, the tibial, and the radial arteries. Furthermore, it is truncated at major organs, such as the kidneys, liver and lungs. For details on parameters and arterial tree data, the reader is referred to Boileau et al. [1], which also contains supplementary data containing the results of the six numerical schemes.

\section{D arterial element}

Each arterial element was modeled as a deformable tube, representing a blood vessel whose mechanical properties are described as a function of the axial coordinate $z$. In large arteries, the relation between pressure $p(\mathrm{~Pa})$, flow $q\left(\mathrm{~m}^{3} \mathrm{~s}^{-1}\right)$ and cross sectional area $\mathrm{A}\left(\mathrm{m}^{2}\right)$ can be modeled using the conservation of mass, the momentum balance equation, and a constitutive law. Here the area is a function of the pressure which in turn is a function of the axial location $z$, and time $t$, i.e. $A=A(p(z, t))$. We further assumed the vessel wall to be impermeable and the blood to be an incompressible Newtonian fluid. Subsequently, by integrating 
the mass and momentum equations over the cross sectional area we obtained the following $1 \mathrm{D}$ equations[9]:

$$
\begin{gathered}
\frac{\partial A}{\partial p} \frac{\partial p}{\partial t}+\frac{\partial q}{\partial z}=0 \\
\frac{\partial q}{\partial t}+\left(1+\delta_{s}\right) \frac{\partial}{\partial z}\left(\frac{q^{2}}{A}\right)+\frac{A}{\rho} \frac{\partial p}{\partial z}=\frac{f}{\rho}
\end{gathered}
$$

where $t$ describes time, $\rho$ is the blood density, $\frac{\partial A}{\partial p}$ is the partial derivative of area to pressure and $f$ is the frictional force per unit length. Following the approach of Boileau et al. [1], a flat velocity profile was used for the approximation of the advection term, which resulted in a Stokes-layer related thickness of $\delta_{s}=0$. Again following Boileau et al. [1], the frictional force per unit length $f$ is given by:

$$
f=-2(\zeta+2) \mu \pi \frac{q}{A}
$$

where $\mu$ is the dynamic viscosity and $\zeta$ is a given constant for a specific velocity profile. For a derivation of Equation 5.3, the reader is referred to Hughes et al. [18] and Sherwin et al. [19]. For the friction force, the velocity profile order $\zeta$ was set to 2 by Boileau et al. [1], which corresponds to a parabolic profile. This mismatch in velocity profiles between the approximation of the advection term and the frictional force can be resolved by using the approximate velocity profile function proposed by Bessems et al. [9], which also captures the possible phase difference between the viscous boundary layer and the central core. However, the approach by Boileau et al. [1] was maintained to be able to compare the results.

The relationship between pressure and area, which accounts for the fluid structure interaction of the system, is described as[1, 20]:

$$
p=p_{e x t}+p_{d}+\frac{\beta}{A_{d}}\left(\sqrt{A}-\sqrt{A_{d}}\right),
$$

with $p_{\text {ext }}$ the external pressure, $p_{d}$ the diastolic pressure and $A_{d}$ the diastolic area. In this study $p_{\text {ext }}$ and $p_{d}$ were set to 0 and $10 \mathrm{kPa}$, respectively[1]. Here, the parameter $\beta(z)$ accounts for the material properties, which is given by $[1,20]$ :

$$
\beta(z)=\frac{4}{3} \sqrt{\pi} E h,
$$

with $E$ and $h$ the Young's modulus and wall thickness, respectively. Using Equation 5.4, area vessel compliance can be written as:

$$
\frac{\partial A}{\partial p}=\left(\frac{2 A_{d}\left(p-p_{e x t}-p_{d}\right)}{\beta}+2 \sqrt{A_{d}}\right) \frac{A_{d}}{\beta}
$$




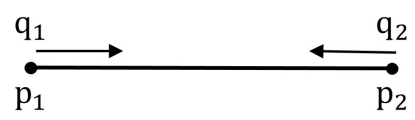

Figure 5.2: Discretization of $1 \mathrm{D}$ line element with $p_{i}$ and $q_{i}$ the nodal pressure and flow, respectively.

\subsubsection{Numerical implementation}

First, the momentum and mass equation of each element were spatially integrated along the vessel axis using the trapezium rule. Thereafter, a second order backward difference scheme was applied to step forward in time. Third, both nodal flows were defined inwards (Figure 5.2). After performing these three steps we arrive at the following $1 \mathrm{D}$ line element:

$$
\left[\begin{array}{ll}
K_{11} & K_{12} \\
K_{21} & K_{22}
\end{array}\right]_{e}^{t}\left[\begin{array}{l}
p_{1} \\
p_{2}
\end{array}\right]_{e}^{t+\Delta t}=\left[\begin{array}{c}
q_{1}^{c} \\
-q_{2}^{c}
\end{array}\right]_{e}^{t+\Delta t}+\left[\begin{array}{l}
f_{1} \\
f_{2}
\end{array}\right]_{e}^{t}=\left[\begin{array}{l}
q_{1} \\
q_{2}
\end{array}\right]_{e}^{t+\Delta t}+\left[\begin{array}{l}
f_{1} \\
f_{2}
\end{array}\right]_{e}^{t}
$$

where column vectors $\underline{p}_{e}$ and $\underline{q}_{e}$ are the nodal point pressures and flows, respectively. Matrix $\mathbf{K}_{e}$ is the element matrix and $f$ is the element right-hand side. Furthermore, $q_{i}^{c}$ denotes the conventional flow definition and $q_{i}$ denotes the inwards defined flows as proposed by Kroon et al. [2], see Figure 5.2. For details of the derivation, the reader is referred to Appendix A. Equation 5.7 can be rewritten in the general form:

$$
\mathbf{K}_{e}^{t, 1 D} \underline{p}_{e}^{t+\Delta t}=\underline{q}_{e}^{t+\Delta t}+\underline{f}_{e}^{t, 1 D} .
$$

\subsubsection{Boundary conditions}

For each simulation, inflow was imposed at the root of the network model, i.e. the ascending aorta. An inflow signal based on the work of Murgo et al. [21] was implemented using a truncated Fourier series of the signal. This is the same inflow signal as used by Boileau et al. [1], and is provided by them in their supplementary material.

At the terminal branches, three-element windkessel elements were modeled[22]. A windkessel element simulates the effect of the peripheral impedance. It consists of a resistor $R_{1}$ in series with both a resistor $R_{2}$ and a compliance $C$ as shown in Figure 5.3. The resistance $R_{1}$ is equal to the characteristic impedance of the terminal branch, minimizing wave reflections for high frequencies[22]. In addition, it holds 


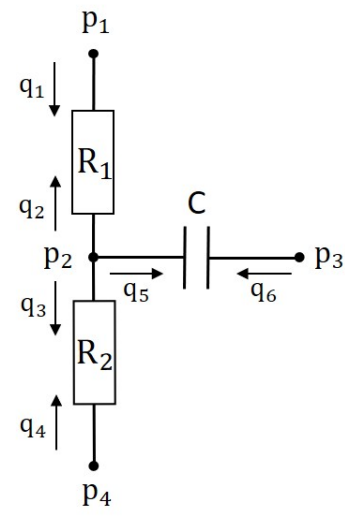

Figure 5.3: Discretization of $0 \mathrm{D}$ windkessel with $p_{i}$ and $q_{i}$ the nodal pressure and flow, respectively. $R_{1}$ is the characteristic impedance, $R_{2}$ the peripheral resistance and $C$ the peripheral compliance.

that $R_{1}+R_{2}=R_{p}$, where $R_{p}$ is the peripheral resistance which is defined by the ratio between the mean arterial pressure and the mean flow through that specific part of the body. Finally, $C$ mimics the peripheral compliance. The windkessel element is given by the following differential equation:

$$
q\left(1+\frac{R_{1}}{R_{2}}\right)+C R_{1} \frac{\partial q}{\partial t}=\frac{p}{R_{2}}+C \frac{\partial p}{\partial t} .
$$

For each subelement of the windkessel element, again two nodal point pressures and two nodal point flows with inward-directing flow were defined (Figure 5.3). The extravascular pressure $\left(p_{4}\right)$ and the venous pressure $\left(p_{3}\right)$ were both taken at $\mathrm{o}$ Pa. Using the same approach as for a 1D line element, the windkessel element can be cast in the same form as Equation 5.8 (see Appendix B):

$$
\mathbf{K}_{e}^{t, 0 D} \underline{p}_{e}^{t+\Delta t}=\underline{f}_{e}^{t, 0 D}+\underline{q}_{e}^{t+\Delta t} .
$$

\subsubsection{Vessel junctions}

At vessel junctions, three 1D arterial elements need to be connected. Since the element flows are defined inwards in the original STM model presented by Kroon et al. [2], the assumption that the $\sum q_{i}=0$, is automatically satisfied. Consequently the nodal pressures are coupled directly without the need for additional coupling 
equations, resulting in a shared node at junctions, as can be seen in Figure 5.1. This continuity of static pressure between the three branches is expressed by:

$$
p_{1}=p_{2}=p_{3} .
$$

However, to be able to compare the STM model to the other five numerical schemes, continuity of total pressure is required at vessel junctions. Therefore, the dynamic part of the pressure should be added to each term in Equation 5.11, yielding:

$$
p_{1}+\frac{1}{2} \rho u_{1}^{2}=p_{2}+\frac{1}{2} \rho u_{2}^{2}=p_{3}+\frac{1}{2} \rho u_{3}^{2},
$$

where $u$ is the mean blood velocity, $\rho$ the blood density, and $p$ and $\frac{1}{2} \rho u^{2}$ are the static and dynamic components of pressure, respectively. Here $\hat{p}=p+\frac{1}{2} \rho u^{2}$ is called the total pressure. The continuity of total pressure can be described by a pressure loss or gain, which results from the difference in mean velocity between branches at junctions:

$$
\begin{aligned}
& p_{1}-p_{2}=\frac{1}{2} \rho\left(u_{2}^{2}-u_{1}^{2}\right), \\
& p_{1}-p_{3}=\frac{1}{2} \rho\left(u_{3}^{2}-u_{1}^{2}\right), \\
& p_{2}-p_{3}=\frac{1}{2} \rho\left(u_{3}^{2}-u_{2}^{2}\right) .
\end{aligned}
$$

To implement these additional pressure differences (losses or gains), a 0D element was introduced and implemented at junctions as shown in Figure 5.1. This 0D junction element is presented in more detail in Figure 5.4. To define the 0D junction element, we denoted one branch as the reference vessel. In this study, the reference vessel carries the total flow entering the element (in case of a bifurcating flow) or leaving the element (in case of a merging flow). However, an arbitrary branch can be chosen. We then assumed that there are no additional pressure differences over the reference vessel, leading to the pressure in the reference vessel being approximately equal to the pressure at the central node $p_{4}$. To prevent an extra pressure drop from occurring, this component was modeled as a small resistance with $J=1 \mathrm{~kg} \mathrm{~m}^{-4} \mathrm{~s}^{-1}$. The value of $J=1 \mathrm{~kg} \mathrm{~m}^{-4} \mathrm{~s}^{-1}$ was chosen such that this value is much smaller than the Poiseuille resistance of the reference vessel. For the other two branches, the pressure differences caused by the addition of the dynamic pressure were defined with respect to the reference vessel: 


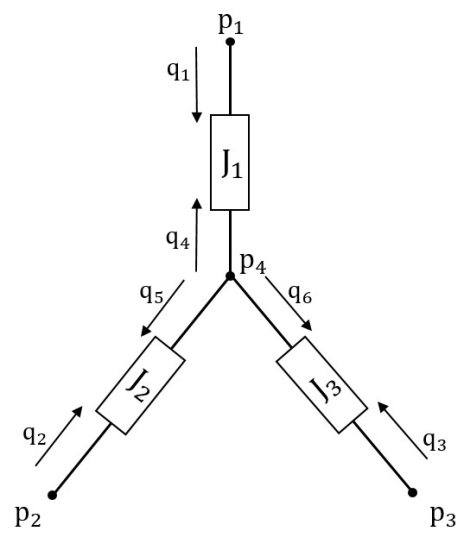

Figure 5.4: junction element

$$
p_{i}-p_{4}=\frac{1}{2} \rho\left(\frac{q_{r}^{2}}{A_{r}^{2}}-\frac{q_{i}^{2}}{A_{i}^{2}}\right),
$$

with $q_{i}$ and $A_{i}$ the flow rate and area of the $i^{t h}$ branch, respectively. Now, each component $J_{i}$ of this junction element relates the pressure of the central node $p_{4}$ to the external pressure nodes $p_{1}, p_{2}$, and $p_{3}$ of the three branches.

After linearization of Equation 5.14 using the Newton-Raphson method we obtained (see Appendix C):

$$
\begin{gathered}
p_{i}^{t+\Delta t}-p_{4}^{t+\Delta t}=\rho \frac{q_{i}^{t}}{\left(A_{i}^{t}\right)^{2}} q_{i}^{t+\Delta t}+ \\
\frac{\rho}{2} \frac{\left(q_{i}^{t}\right)^{2}}{\left(A_{i}^{t}\right)^{2}}+\frac{\rho}{2} \frac{\left(q_{r}^{t}\right)^{2}}{\left(A_{r}^{t}\right)^{2}}, i=1,2,3,
\end{gathered}
$$

where superscript $t$ is the approximation of the previous timestep and $t+\Delta t$ the approximation of the current timestep. This can be summarized to the general form:

$$
p_{i}^{t+\Delta t}-p_{4}^{t+\Delta t}=J_{i}^{t} q_{i}^{t+\Delta t}+g_{i}^{t} \quad i=1,2,3,
$$

with $g_{i}$ the last two terms of the right-hand side of Equation 5.15. 
We then divided Equation 5.16 by $J_{i}$ resulting in:

$$
\frac{1}{J_{i}}\left(p_{i}^{t+\Delta t}-p_{4}^{t+\Delta t}\right)=q_{i}^{t+\Delta t}+f_{i}^{t}, \quad \quad \mathbf{i}=\mathbf{1 , 2 , 3},
$$

with $f_{i}^{t}=\frac{g_{i}^{t}}{J_{i}}$. Now component $J_{1}$ in Figure 5.4 could be cast into the form:

$$
\left[\begin{array}{cc}
\frac{1}{J_{1}} & -\frac{1}{J_{1}} \\
-\frac{1}{J_{1}} & \frac{1}{J_{1}}
\end{array}\right]_{e}^{t}\left[\begin{array}{l}
p_{1} \\
p_{4}
\end{array}\right]_{e}^{t+\Delta t}=\left[\begin{array}{c}
q_{1}^{c} \\
-q_{4}^{c}
\end{array}\right]_{e}^{t+\Delta t}+\left[\begin{array}{c}
f_{1} \\
-f_{1}
\end{array}\right]_{e}^{t}=\left[\begin{array}{l}
q_{1} \\
q_{4}
\end{array}\right]_{e}^{t+\Delta t}+\left[\begin{array}{c}
f_{1} \\
-f_{1}
\end{array}\right]_{e}^{t},
$$

where $q_{i}^{c}$ once gain denotes the conventional notation and $q_{i}$ the notation as presented by Kroon et al. [2].

Similarly, component $J_{2}$ and $J_{3}$ were cast into the same form. By assembling the three junction components, the total element matrix is:

$$
\begin{aligned}
& {\left[\begin{array}{cccc}
\frac{1}{J_{1}} & 0 & 0 & -\frac{1}{J_{1}} \\
0 & \frac{1}{J_{2}} & 0 & -\frac{1}{J_{2}} \\
0 & 0 & \frac{1}{J_{3}} & -\frac{1}{J_{3}} \\
-\frac{1}{J_{1}} & -\frac{1}{J_{2}} & -\frac{1}{J_{3}} & \frac{1}{J_{1}}+\frac{1}{J_{2}}+\frac{1}{J_{3}}
\end{array}\right]_{e}^{t}\left[\begin{array}{c}
p_{1} \\
p_{2} \\
p_{3} \\
p_{4}
\end{array}\right]_{e}^{t+\Delta t}} \\
& =\left[\begin{array}{c}
q_{1} \\
q_{2} \\
q_{3} \\
-q_{4}-q_{5}-q_{6}
\end{array}\right]_{e}^{t+\Delta t}+\left[\begin{array}{c}
f_{1} \\
f_{2} \\
f_{3} \\
-f_{1}-f_{2}-f_{3}
\end{array}\right]_{e}^{t},
\end{aligned}
$$

with $-q_{4}-q_{5}-q_{6}=0$ due to conservation of mass. Finally, the $0 \mathrm{D}$ junction element (defined by Equation 5.19) was cast into the same form as the 1D line elements (Equation 5.8) and the 0D windkessel element (Equation 5.10):

$$
\mathbf{K}_{e}^{t, 0 D} \underline{p}_{e}^{t+\Delta t}=\underline{f}_{e}^{t, 0 D}+\underline{q}_{e}^{t+\Delta t} .
$$

\subsubsection{Assembling all elements and Numerical method}

Noticing that the element matrices, i.e. Equations 5.8, 5.10, and 5.20, were all cast in the same form, assembly of the large system of equations involved summation of all $1 \mathrm{D}$ element equations and $0 \mathrm{D}$ element equations. The assembly process is here demonstrated for two line elements in series. Consider Element 1 with pressure nodes $p_{1}$ and $p_{2}$ and flows $q_{1}$ and $q_{2}$, and Element 2 with pressure nodes $p_{2}$ and $p_{3}$ and flows $q_{3}$ and $q_{4}$, respectively. The two element matrices of the two line elements defined by Equation 5.7, can be assembled to the following system: 


$$
\begin{aligned}
& {\left[\begin{array}{ccc}
K_{11}^{1} & K_{12}^{1} & 0 \\
K_{21}^{1} & K_{22}^{1}+K_{11}^{2} & K_{12}^{2} \\
0 & K_{21}^{2} & K_{22}^{2}
\end{array}\right]_{e}^{t}\left[\begin{array}{c}
p_{1} \\
p_{2} \\
p_{3}
\end{array}\right]_{e}^{t+\Delta t}} \\
& =\left[\begin{array}{c}
q_{1} \\
-q_{2}+q_{3} \\
-q_{4}
\end{array}\right]_{e}^{t+\Delta t}+\left[\begin{array}{c}
f_{1}^{1} \\
f_{2}^{1}+f_{1}^{2} \\
f_{2}^{2}
\end{array}\right]_{e}^{t}
\end{aligned}
$$

where the superscripts of $K_{i}$ and $f_{i}$ represent the elements they belong to. Conservation of mass requires the outflow of element $1\left(q_{2}\right)$ to be equal to the inflow of element $2\left(q_{3}\right)$, resulting in $-q_{2}+q_{3}=0$. Therefore, the internal node in this example has value of 0 and only the flows $q_{1}$ and $q_{4}$ (boundary conditions) are unknown. At the inlet of the arterial tree, the nodal flow was prescribed and for terminal nodes a constant pressure was prescribed (essential boundary condition), resulting in only one degree of freedom for every node. The final assembled matrix containing the $1 \mathrm{D}$ line, $0 \mathrm{D}$ windkessel and $0 \mathrm{D}$ junction elements is given by:

$$
\mathbf{K}^{t} \underline{p}^{t+\Delta t}=\underline{f}^{t}+\underline{q}^{e x, t+\Delta t}
$$

The system was solved using the second order backward difference scheme for time discretization, with a timestep of $0.5 \mathrm{~ms}$ and element size of $0.5 \mathrm{~cm}[2]$. The finite element package SEPRAN (version 0610) was used to solve the system, while Matlab R2016b (The MathWorks, Inc, Natick, Massachusetts) was used for pre- and postprocessing. After calculation of pressures and flows, the simulation proceeded to the next timestep. The process was repeated until cardiac cycle time was reached. At this point, the nodal point maximum relative root-mean-squared norm of both pressure and flow, denoted $\varepsilon_{p}$ and $\varepsilon_{q}$ respectively, were calculated compared to the previous cardiac cycle. Each simulation was run for 10 cycles, resulting in a convergence norm $\varepsilon<10^{-3}$.

The following simulations were run for the ADAN56 benchmark case: 1) The original STM method (STM) 2) STM + new 0D junction element (STM-J) 3) the Finite Element Method scheme (FEM) presented in Boileau et al. [1]. The other four schemes shown in Boileau et al. [1] that already have continuity of total pressure at vessel junctions gave consistent results with the FEM method and are therefore not shown. The results of the five other benchmark cases are not shown as the STM scheme already showed good agreement compared to the other methods, even without the addition of the dynamic part of the pressure, and because these cases were hardly effected by the addition of the new $0 \mathrm{D}$ junction element, as expected. 
First, flow rate and pressure waveforms are shown for the Aortic Arch I, the Right Internal Carotid, the Right Renal and the Right Anterior Tibial. These locations were chosen to show the effect of adding the dynamic pressure when moving more distal from the heart, both upwards and downwards. We then calculate a variety of "errors" that are calculated to compare the results using the FEM as the golden standard: $\mathcal{E}_{p}^{R M S}$ and $\mathcal{E}_{q}^{R M S}$ are the root mean square relative error in pressure and flow, $\mathcal{E}_{p}^{M A X}$ and $\mathcal{E}_{q}^{M A X}$ are the maximum relative errors in pressure and flow, $\mathcal{E}_{p}^{S Y S}$ and $\mathcal{E}_{q}^{S Y S}$ are the errors in systolic pressure and flow and $\mathcal{E}_{p}^{D I A S}$ and $\mathcal{E}_{q}^{D I A S}$ are the errors in diastolic pressure and flow, respectively. These are calculated with the following equations:

$$
\begin{aligned}
& \mathcal{E}_{p}^{R M S}=\sqrt{\frac{1}{n} \sum_{i=1}^{n}\left(\frac{p_{i}^{S T M}-p_{i}^{F E M}}{p_{i}^{F E M}}\right)^{2},} \\
& \mathcal{E}_{q}^{R M S}=\sqrt{\frac{1}{n} \sum_{i=1}^{n}\left(\frac{q_{i}^{S T M}-q_{i}^{F E M}}{\max \left(q_{i}^{F E M}\right)}\right)^{2}}, \\
& \mathcal{E}_{p}^{M A X}=\max _{i} \mid \frac{p_{i}^{S T M}-p_{i}^{F E M}}{p_{i}^{F E M} \mid}, \\
& \mathcal{E}_{q}^{M A X}=\max _{i}\left|\frac{q_{i}^{S T M}-q_{i}^{F E M}}{\max \left(q_{i}^{F E M}\right)}\right|, \\
& \mathcal{E}_{p}^{S Y S}=\frac{\max \left(p^{S T M}\right)-\max \left(p^{F E M}\right)}{\max \left(p^{F E M}\right)}, \\
& \mathcal{E}_{q}^{S Y S}=\frac{\max \left(q^{S T M}\right)-\max \left(q^{F E M}\right)}{\max \left(q^{F E M}\right)}, \\
& \mathcal{E}_{q}^{D I A S}=\frac{\min \left(q^{S T M}\right)-\min \left(q^{F E M}\right)}{\min \left(q^{F E M}\right)} \\
& \mathcal{E}_{p}^{D I A S}=\frac{\min \left(p^{S T M}\right)-\min \left(p^{F E M}\right)}{\min \left(p^{F E M}\right)}
\end{aligned}
$$

with value $n$ the maximum number of timesteps within one cardiac cycle. Finally, we show the propagation of the systolic, diastolic, and mean pressure along the aorta, because this captures the general physiological features of pulse wave propagation. 


\subsection{Results}

Figure 5 shows the flow rate and pressure waveforms of the four different positions in the ADAN 56 network. Though in each panel of Figure 5 the waveforms as derived by STM, STM-J and FEM are plotted, the latter two signals can often not be discriminated, indicating their good agreement. This good agreement can also be seen when looking at the errors in Table 5.1. In the original STM model the root mean square relative errors $\mathcal{E}^{R M S}$ ranged from $0.51 \%$ to $4.10 \%$, while with the extended STM-J model the root mean square relative errors $\mathcal{E}^{R M S}$ have a fourfold reduction and range from $0.12 \%$ to $0.67 \%$. Furthermore, the systolic- and diastolic pressure are in better agreement for the STM-J model, with a fourfold error reduction in the Aortic Arch I. This error reduction increases for more distal vessels, as seen for the Right Anterior Tibial, where the relative systolic error decreases tenfold.

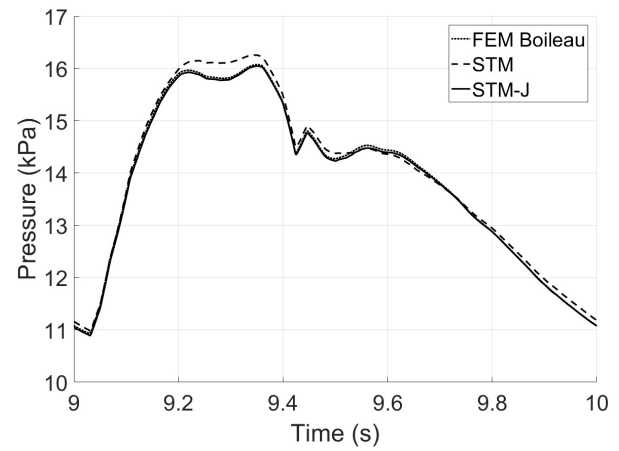

(a) Pressure in Aortic Arch I

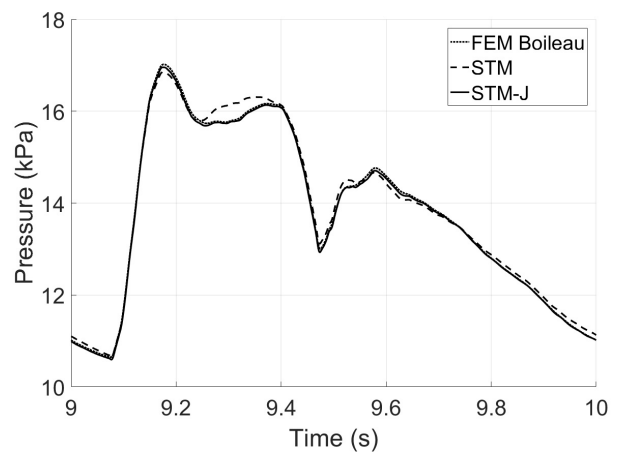

(c) Pressure in Right Internal Carotid

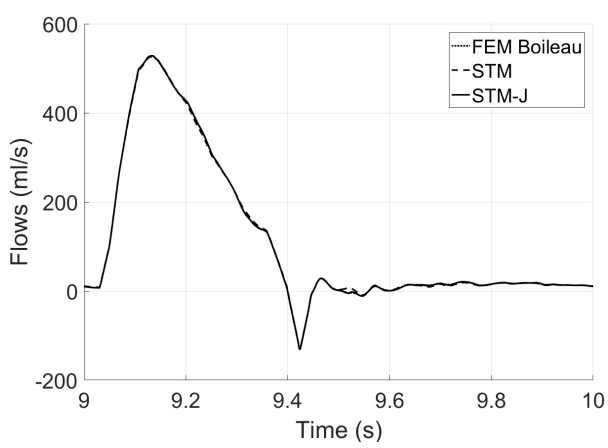

(b) Flow Rate in Aortic Arch I

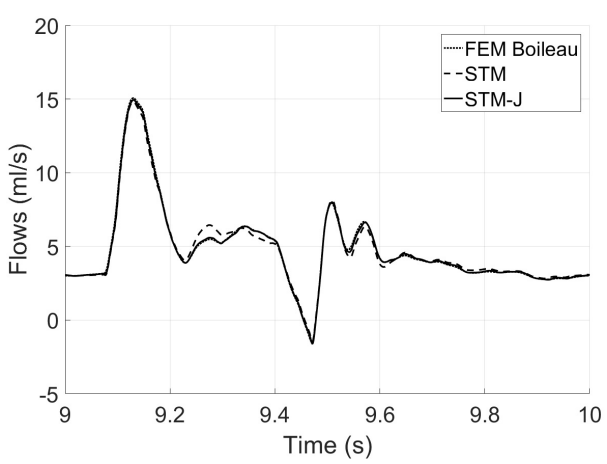

(d) Flow Rate in Right Internal Carotid 


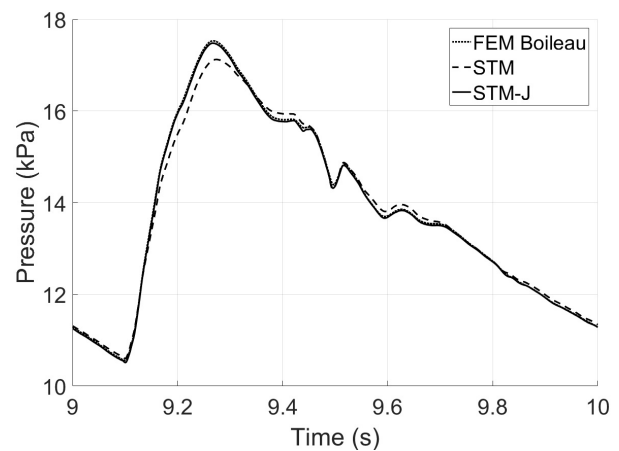

(e) Pressure in Right Renal

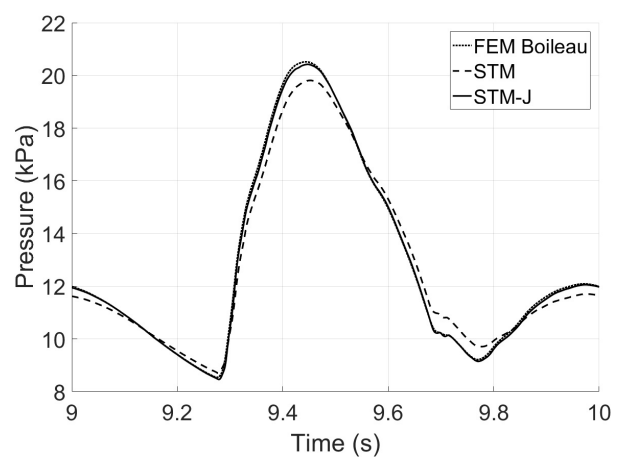

(g) Pressure in Right Anterior Tibial

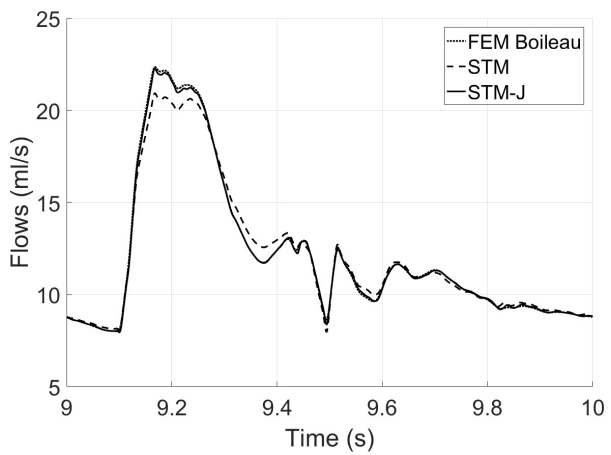

(f) Flow Rate in Right Renal

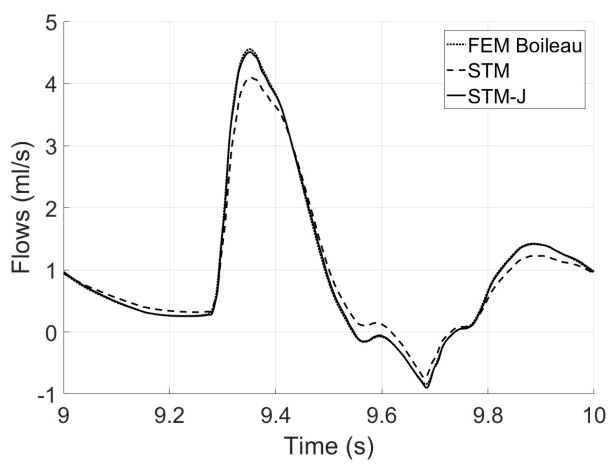

(h) Flow Rate in Right Anterior Tibial

Figure 5.5: Pressure and flow rate waveforms, comparing the Finite Element Method (FEM), Simplified Trapezoidal Rule Method (STM) and STM with junction (STM-J) 
Table 5.1: Relative error with respect to the Finite Element method at the midpoint of the vessels in percent.

\begin{tabular}{|c|c|c|c|c|c|c|c|c|c|}
\hline$\overline{\text { Arterial }}$ & Method & $\begin{array}{l}\mathcal{E}_{p}^{R M S} \\
(\%)\end{array}$ & $\begin{array}{l}\mathcal{E}_{p}^{M A X} \\
(\%)\end{array}$ & $\begin{array}{l}\mathcal{E}_{p}^{S Y S} \\
(\%)\end{array}$ & $\begin{array}{l}\mathcal{E}_{p}^{D I A S} \\
(\%)\end{array}$ & $\begin{array}{l}\mathcal{E}_{q}^{R M S} \\
(\%)\end{array}$ & $\begin{array}{l}\mathcal{E}_{q}^{M A X} \\
(\%)\end{array}$ & $\begin{array}{l}\mathcal{E}_{q}^{S Y S} \\
(\%)\end{array}$ & $\begin{array}{l}\mathcal{E}_{q}^{D I A S} \\
(\%)\end{array}$ \\
\hline \multirow[t]{2}{*}{ Aortic Arch I } & STM & 0.83 & 1.87 & 1.14 & 0.50 & 0.51 & 1.94 & -0.17 & 0.34 \\
\hline & STM-J & 0.25 & 0.55 & -0.16 & -0.34 & 0.12 & 0.41 & 0.13 & 1.38 \\
\hline \multirow[t]{2}{*}{ Right Internal Carotid } & STM & 0.89 & 2.35 & -1.00 & 0.13 & 1.98 & 6.41 & -1.51 & -3.11 \\
\hline & STM-J & 0.27 & 0.69 & -0.38 & -0.49 & 0.56 & 2.23 & 0.82 & 6.90 \\
\hline \multirow[t]{2}{*}{ Right Renal } & STM & 1.11 & 3.09 & -2.31 & 0.39 & 2.25 & 6.90 & -6.35 & -0.47 \\
\hline & STM-J & 0.24 & 0.63 & -0.29 & -0.44 & 0.41 & 1.57 & -0.33 & -0.74 \\
\hline \multirow[t]{2}{*}{ Right Anterior Tibial } & STM & 3.39 & 6.95 & -3.44 & -1.90 & 4.10 & 12.54 & -10.02 & -11.13 \\
\hline & STM-J & 0.67 & 2.99 & -0.47 & -0.94 & 0.64 & 2.68 & -1.04 & -7.16 \\
\hline
\end{tabular}




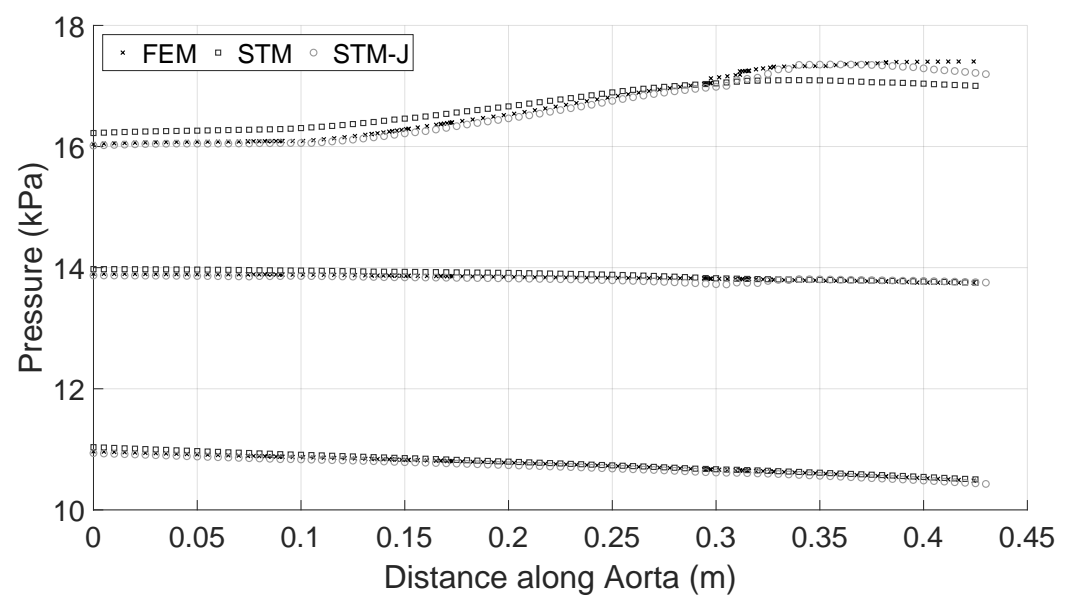

Figure 5.6: Systolic, mean, and diastolic pressure along the aorta for the Finite Element Method (FEM), the Simplified trapezium Method (STM) and the Simplified Trapezium Method with new Junction element (STM-J).

Figure 5.6 shows the propagation of the systolic, mean and diastolic pressure along the aorta. While the STM shows an overestimation of $1 \%$ of the systolic pressure in the beginning of the aorta and an underestimation of $3 \%$ when moving more distally, the new STM-J model resolves this error for the systolic pressure. This can be seen by a relative error that is lower than $0.5 \%$ when comparing the systolic pressure of the STM-J model along the aorta with the systolic pressure of the FEM model.

\subsection{Discussion}

The aim of this study was to introduce a new $0 \mathrm{D}$ junction element to incorporate the dynamic part of the pressure in the already existing STM model proposed by Kroon et al. [2]. Using the new coupling strategy described in Section 5.2, the total pressure $\left(p+\frac{1}{2} \rho u^{2}\right)$ was constrained at vessel junctions without the need of expanding the global matrix with Lagrange multipliers or introducing a penalty function as previously published by Carson et al. [3]. After implementing the new 0D junction element the pressure and flow waveforms showed good agreement with the results of the five other numerical schemes described by Boileau et al. [1] (see Figure 5) that already had incorporated dynamic pressure at vessel junctions. 
By following the inward-directed flow approach as described by Kroon et al. [2] we showed the flexibility of the STM model and the relative ease of implementing a new element. By casting every element in the same form as in Equation 5.22 and defining flow inwards at element level, no extra coupling equations were needed between elements, and computational speed and efficiency were maintained. This approach was recently also used by Qureshi et al. [23] who modeled the pulmonary and venous circulation. Moreover, this approach can also easily be used to implement other vascular concepts with nonlinear pressure-flow rate relations, such as a stenosis, a heart pump or the calf muscle pump function[5]. Although we demonstrated the ease of adding the dynamic part of the pressure to the STM method, one might argue the importance of adding the dynamic part. The influence of pressure losses at junctions becomes of more importance when the magnitude of the blood flow velocity is increased. Mynard et al. [24] estimated a pressure loss of $1 \mathrm{mmHg}(\sim 0.5 \mathrm{kPa})$ after three typical diverging or converging junctions under resting circumstances with a maximum velocity of $0.5 \mathrm{~m} \mathrm{~s}^{-1}$. During exercise, with a maximum aortic blood flow velocity of $2.0 \mathrm{~m} \mathrm{~s}^{-1}$, they estimated a pressure loss of $17 \mathrm{mmHg}(\sim 2.3 \mathrm{kPa})$ after three junctions. This dependence on blood flow velocity and number of junctions is even larger in the pulmonary arterial and the venous network, which consists of many generations of junctions[23].

Mynard et al. [24] showed that both methods of coupling (with or without the dynamic part) did not result in a reliable estimation of the pressure at junctions compared with 3D simulations and that neither method could be judged as generally superior to the other. While conservation of dynamic pressure will affect the pressure losses at vascular junctions, an extra energy dissipation, that depends on the junction geometry and flow conditions, is also present. This dissipation may counterbalance the pressure losses from the addition of the dynamic part of the pressure. The extra pressure loss $K_{1,2}\left(\frac{1}{2} \rho u_{c o m}^{2}\right)$ is caused by a change of direction of flow and will result in an extra pressure loss due to the formation of vortices at the entrance region of a bifurcation[23]. Therefore, Equation 5.13 should be written as:

$$
p_{1}-p_{2}=\frac{1}{2} \rho\left(u_{2}^{2}-u_{1}^{2}\right)+K_{1,2}\left(\frac{1}{2} \rho u_{\text {com }}^{2}\right)
$$

with $u_{\text {com }}$ the velocity in the common branch and $K_{1,2}$ a constant that is dependent on the junction geometry and flow conditions. Mynard et al. [24] proposed a method for estimating the constant $K_{1,2}$ and thus the calculation of the extra pressure losses at junctions. Although not modeled in the current $0 \mathrm{D}$ junction element, this extra pressure loss can easily be added to the current element following the approach proposed in this article.

Though the current $0 \mathrm{D}$ junction element only connects three line elements, it can 
easily be expanded to junctions with more than three branches. However, because these junctions with more than three branches are mostly found in smaller vessels, the assumptions that are made for 1D modeling of the larger blood vessels, e.g. Newtonian fluid, possibly no longer hold[9]. Extending the junction element to more branches therefore has to be done with care.

Choosing the right coupling strategy is shown to be important on the wave propagation. The possible addition of dynamic part of the pressure $\left(\frac{1}{2} \rho u^{2}\right)$ and the extra pressure loss $\left(K_{1,2}\left(\frac{1}{2} \rho u_{c o m}^{2}\right)\right)$ must be evaluated for every application. While for simple bifurcations the differences between coupling strategies are negligible, these differences become larger for arterial networks with an increasing number of generations and with increasing blood velocity $[1,24]$. However, the influence of the pressure coupling can be much smaller compared to changes by choosing a different velocity profile, a different boundary inflow, a different constitutive law or the implementation of visco-elastic wall behaviour[25]. Because Newtonian fluid is assumed in most 1D schemes (including ours), blood rheology effects including blood shear-thinning are neglected[26]. Since we were only interested in implementing a dynamic pressure element and comparing this with already existing models, the lack of such a rheology model is not of importance for our study. Moreover, the geometry used in our study consists of vessels of a large calibre, where blood shear thinning is not as important, because of high shear regions[26]. Although it is possible to make the model more complex or less complex [25-27], it is very important to evaluate model choices, such as the inclusion of rheology or visco-elastic wall behaviour, depending on the models application.

\subsection{Conclusion}

The implementation of a 0D junction element in the Simplified Trapezium rule Method enabled incorporation of the dynamic part of the pressure at vessel junctions. Using the approach by Kroon et al. [2], flexible coupling between 0D and 1D elements is possible and nonlinear pressure-flow equations can be easily incorporated. The extended STM model shows good agreement with the five other schemes shown by Boileau et al. [1], while maintaining computational speed and efficiency. 


\section{Appendix}

\section{A 1D Line Element Equations}

First of all, recall the momentum equation (Equation 5.2):

$$
\frac{\partial q}{\partial t}+\left(1+\delta_{s}\right) \frac{\partial}{\partial z}\left(\frac{q^{2}}{A}\right)+\frac{A}{\rho} \frac{\partial p}{\partial z}=\frac{f}{\rho},
$$

with

$$
f=-2(\zeta+2) \mu \pi \frac{q}{A}
$$

Also recall the mass equation:

$$
C_{A} \frac{\partial p}{\partial t}+\frac{\partial q}{\partial z}=0
$$

with $C_{A}=\frac{\partial A}{\partial p}$.

If we set $\zeta=2$, and $\delta_{s}=0$ in Equations 5.32 and 5.33, corresponding to[1], these equations reduce to:

$$
\frac{\rho}{A} \frac{\partial q}{\partial t}+\frac{\partial p}{\partial z}=-\frac{8 \mu \pi q}{A^{2}}-\frac{\rho}{A} \frac{\partial}{\partial z}\left(\frac{q^{2}}{A}\right)
$$

To discretize and linearize this $1 \mathrm{D}$ momentum equation (Equation 5.35) and the conservation of mass equation (Equation 5.1), the vessels are first divided into non-overlapping two-noded elements. Therefore it holds that:

$$
\int(\bullet) d z=\sum_{e=1}^{N_{e}} \int_{e}(\bullet) d z
$$

where $N_{e}$ is the number of elements. 
The trapezium rule is then used to spatially integrate both the conservation of mass and the momentum equation from node 1 to node 2 . Note that the flows are here defined conventionally meaning that both flows within the element are directed from node 1 to node 2 . The integration over the element domain $e$, using the trapezium rule yields:

$$
\begin{array}{r}
\int_{e} C_{A} \frac{\partial p}{\partial t} d z \approx \frac{\Delta z_{e}}{2}\left(C_{A, 1} \frac{\partial p_{1}}{\partial t}+C_{A, 2} \frac{\partial p_{2}}{\partial t}\right), \\
\int_{e}\left(\frac{\partial p}{\partial z}\right) d z \approx\left(p_{2}-p_{1}\right), \\
\int_{e} \frac{\rho}{A} \frac{\partial q}{\partial t} d z \approx \frac{\Delta z_{e}}{2}\left(\frac{\rho}{A_{1}} \frac{\partial q_{1}^{c}}{\partial t}+\frac{\rho}{A_{2}} \frac{\partial q_{2}^{c}}{\partial t}\right), \\
\int_{e} \frac{\partial q}{\partial z} d z=\left(q_{2}^{c}-q_{1}^{c}\right), \quad \int_{e} h d z \approx \frac{\Delta z_{e}}{2}\left(h_{1}+h_{2}\right), \\
h_{i}=\left\{-\frac{8 \mu \pi q}{A^{2}}-\frac{\rho}{A} \frac{\partial}{\partial z}\left(\frac{q^{2}}{A}\right)\right\}
\end{array}
$$

Here $\Delta z_{e}$ is the element length and the superscript $c$ denotes the use of the conventional definition of flows. Note: at this point thus different from the definition used during our numerical implementation. In matrix form, Equation 5.37 becomes:

$$
\begin{array}{r}
{\left[\begin{array}{l}
C_{A, 1} \frac{\Delta z_{e}}{2} \\
C_{A, 2} \frac{\Delta z_{e}}{2}
\end{array}\right]^{T} \frac{\partial \underline{p}_{e}}{\partial t}+\left[\begin{array}{l}
-1 \\
+1
\end{array}\right]^{T} \underline{q}_{e}^{c}=0} \\
{\left[\begin{array}{c}
\frac{\rho}{A_{1}} \frac{\Delta z_{e}}{2} \\
\frac{\rho}{A_{1}} \frac{\Delta z_{e}}{2}
\end{array}\right]^{T} \frac{\partial \underline{q}_{e}^{c}}{\partial t}+\left[\begin{array}{l}
-1 \\
+1
\end{array}\right]^{T} \underline{p}_{e}=\left(h_{1}+h_{2}\right) \frac{\Delta z_{e}}{2}}
\end{array}
$$

where $\underline{p}_{e}=\left[p_{1}, p_{2}\right]^{T}$ and $\underline{q}_{e}^{c}=\left[q_{1}^{c}, q_{2}^{c}\right]^{T}$ contain nodal point pressures and flows of the element, respectively. We now apply a second-order backward differentiation scheme for the time derivatives with timestep $\Delta t$. Now we can rewrite Equation 5.38 in the following matrix notation:

$$
\mathbf{F}_{\mathbf{e}} \underline{p}_{e}^{t+\Delta t}+\mathbf{G}_{e}^{c} \underline{q}_{e}^{c, t+\Delta t}=\underline{h}_{e},
$$

where:

$$
\mathbf{F}_{\mathbf{e}}=\left[\begin{array}{cc}
\frac{3}{2 \Delta t} \frac{\Delta z}{2} C_{A, 1}^{t} & \frac{3}{2 \Delta t} \frac{\Delta z}{2} C_{A, 2}^{t} \\
-1 & +1
\end{array}\right]
$$




$$
\begin{gathered}
\mathbf{G}_{\mathbf{e}}^{\mathbf{c}}=\left[\begin{array}{cc}
-1 & -1 \\
\frac{3}{2 \Delta t} \frac{\Delta z}{2} \frac{\rho}{A_{1}^{t}} & -\frac{3}{2 \Delta t} \frac{\Delta z}{2} \frac{\rho}{A_{2}^{t}}
\end{array}\right] \\
\underline{h_{e}}=\left[\begin{array}{c}
0 \\
\left(h_{1}^{t}+h_{2}^{t}\right) \frac{\Delta z}{2}
\end{array}\right]+\left[\begin{array}{c}
\frac{\Delta z}{2}\left(\frac{2}{\Delta t} C_{A, 1}^{t} p_{1}^{t}-\frac{1}{2 \Delta t} C_{A, 1}^{t} p_{1}^{t-\Delta t}\right) \\
\frac{\Delta z}{2}\left(\frac{2}{\Delta t} \frac{\rho}{A_{1}^{t}} q_{1}^{t}-\frac{1}{2 \Delta t} \frac{\rho}{A_{1}^{t}} q_{1}^{t-\Delta t}\right)
\end{array}\right] \\
+\left[\begin{array}{c}
\frac{\Delta z}{2}\left(\frac{2}{\Delta t} C_{A, 2}^{t} p_{2}^{t}-\frac{1}{2 \Delta t} C_{A, 2}^{t} p_{2}^{t-\Delta t}\right) \\
\frac{\Delta z}{2}\left(\frac{2}{\Delta t} \frac{\rho}{A_{2}^{t}} q_{2}^{t}-\frac{1}{2 \Delta t} \frac{\rho}{A_{2}^{t}} q_{2}^{t-\Delta t}\right)
\end{array}\right]
\end{gathered}
$$

Next, we make a switch to the discretization as depicted in Figure 5.2, where both flows are defined inwards, that is $q_{1}=q_{1}^{c}$ and $q_{2}=-q_{2}^{c}$. Now we can rewrite Equation 5.39 in the following matrix notation form:

$$
\mathbf{F}_{\mathbf{e}} \underline{p}_{e}^{t+\Delta t}+\mathbf{G}_{e} \underline{q}_{e}^{t+\Delta t}=\underline{h}_{e}
$$

with

$$
\mathbf{G}_{\mathbf{e}}=\left[\begin{array}{ll}
G_{e, 11}^{c} & -G_{e, 12}^{c} \\
G_{e, 21}^{c} & -G_{e, 22}^{c}
\end{array}\right], \quad \underline{q}_{e}=\left[\begin{array}{c}
q_{1}^{c} \\
-q_{2}^{c}
\end{array}\right]=\left[\begin{array}{c}
q_{1} \\
q_{2}
\end{array}\right]
$$

After separation of matrix $\mathbf{G}_{\mathbf{e}}$ from flow column $\underline{q}_{e}^{t+\Delta t}$, the 1D line element equations finally read:

$$
\left[-\mathbf{G}_{e}^{-1} \mathbf{F}_{e}\right] \underline{p}_{e}^{t+\Delta t}=\underline{q}_{e}^{t+\Delta t}+\left[-\mathbf{G}_{e}^{-1} \underline{h}_{e}\right] .
$$

Which is now cast into the same general form:

$$
\mathbf{K}_{e}^{t, 1 D} \underline{p}_{e}^{t+\Delta t}=\underline{q}_{e}^{t+\Delta t}+\underline{f}_{e}^{t, 1 D} .
$$

\section{B OD Windkessel Element Equations}

The three elements in Figure 5.3 are described by two resistors and one compliance. In matrix form, the equations for these elements become:

$$
\begin{aligned}
& R_{1}\left[\begin{array}{cc}
1 & -1 \\
-1 & 1
\end{array}\right]\left[\begin{array}{l}
p_{1} \\
p_{2}
\end{array}\right]=\left[\begin{array}{l}
q_{1} \\
q_{2}
\end{array}\right], \\
& R_{2}\left[\begin{array}{cc}
1 & -1 \\
-1 & 1
\end{array}\right]\left[\begin{array}{l}
p_{2} \\
p_{3}
\end{array}\right]=\left[\begin{array}{l}
q_{3} \\
q_{4}
\end{array}\right], \\
& C\left[\begin{array}{cc}
1 & -1 \\
-1 & 1
\end{array}\right]\left[\begin{array}{l}
\frac{\partial p_{2}}{\partial t} \\
\frac{\partial p_{4}}{\partial t}
\end{array}\right]=\left[\begin{array}{l}
q_{5} \\
q_{6}
\end{array}\right] .
\end{aligned}
$$


These element can be assembled to

$$
\mathbf{C}_{e} \frac{\partial \underline{p}_{e}}{\partial t}+\mathbf{R}_{e}^{r} \underline{p}_{e}=\underline{q}_{e},
$$

with:

$$
\begin{gathered}
\mathbf{C}_{e}=\left[\begin{array}{cccc}
0 & 0 & 0 & 0 \\
0 & C & -C & 0 \\
0 & 0 & 0 & 0 \\
0 & -C & C & 0
\end{array}\right], \\
\mathbf{R}_{e}^{r}=\left[\begin{array}{cccc}
\frac{1}{Z} & -\frac{1}{Z} & 0 & 0 \\
-\frac{1}{Z} & \frac{1}{Z}+\frac{1}{R} & 0 & -\frac{1}{R} \\
0 & -\frac{1}{R} & 0 & \frac{1}{R} \\
0 & 0 & 0 & 0
\end{array}\right],
\end{gathered}
$$

where $\underline{p}_{e}=\left[p_{1}, p_{2}, p_{3}, p_{4}\right]^{T}$, and $\underline{q}_{e}=\left[q_{1}, q_{2}+q_{3}+q_{5}, q_{4}, q_{6}\right]^{T}$.

Application of the second order backward differentiation scheme on Equation $5 \cdot 50$ results in:

$$
\left(\frac{3}{2 \Delta t} \mathbf{C}_{e}+\mathbf{R}_{e}^{r}\right) \underline{p}_{e}^{t+\Delta t}=\left(\mathbf{C}_{e}\left(-\frac{-2}{\Delta t} \underline{p}_{e}^{t}-\frac{1}{2 \Delta t} \underline{p}_{e}^{t-\Delta t}\right)\right)+\underline{q}_{e}^{t+\Delta t},
$$

which is now cast into the same general form:

$$
\mathbf{K}_{e}^{t, 0 D} \underline{p}_{e}^{t+\Delta t}=\underline{f}_{e}^{t, 0 D}+\underline{q}_{e}^{t+\Delta t} .
$$

\section{C Newton-Raphson Linearization}

The linearization of Equation 5.14 is performed using the Newton-Raphson method. This method is used to step forward in time and uses the current timestep to estimate the pressure of the next timestep. At timestep $t$, Equation 5.14 gives:

$$
p_{i}^{t}-p_{4}^{t}=\frac{1}{2} \rho\left(\frac{\left(q_{r}^{t}\right)^{2}}{\left(A_{r}^{t}\right)^{2}}-\frac{\left(q_{i}^{t}\right)^{2}}{\left(A_{i}^{t}\right)^{2}} .\right.
$$

The right-handside can than be written as a function with variable $q_{i}$, which results in:

$$
p_{i}^{t}-p_{4}^{t}=f\left(q_{i}^{t}\right)
$$

If we use this equation to step forward in time, this results in:

$$
p_{i}^{t+\Delta t}-p_{4}^{t+\Delta t}=f\left(q_{i}^{t+\Delta t}\right),
$$


with $\Delta t$ the timestep. We now want to apply the Newton-Raphson method for the right-handside, which uses the previous timestep and estimates the difference with the next timestep:

$$
p_{i}^{t+\Delta t}-p_{4}^{t+\Delta t}=f\left(q_{i}^{t}\right)+\frac{\partial f\left(q_{i}^{t}\right)}{\partial q_{i}^{t}}\left(q_{i}^{t+\Delta t}-q_{i}^{t}\right),
$$

here, the higher order terms are omitted.

Using this Newton-Raphson method, Equation 5.55 can finally be rewritten to Equation 5.15:

$$
\begin{array}{r}
p_{i}^{t+\Delta t}-p_{4}^{t+\Delta t}=\rho \frac{q_{i}^{t}}{\left(A_{i}^{t}\right)^{2}} q_{i}^{t+\Delta t}+ \\
\frac{\rho}{2} \frac{\left(q_{i}^{t}\right)^{2}}{\left(A_{i}^{t}\right)^{2}}+\frac{\rho}{2} \frac{\left(q_{r}^{t}\right)^{2}}{\left(A_{r}^{t}\right)^{2}} \\
\text { with } \mathrm{i}=\mathbf{1 , 2 , 3} .
\end{array}
$$




\section{References}

1. Boileau, E., Nithiarasu, P., Blanco, P. J. et al. A benchmark study of numerical schemes for onedimensional arterial blood flow modelling. International Journal for Numerical Methods in Biomedical Engineering 30, n/a (2015).

2. Kroon, W., Huberts, W., Bosboom, M. \& van de Vosse, F. A numerical method of reduced complexity for simulating vascular hemodynamics using coupled $\mathrm{OD}$ lumped and $\mathrm{1} D$ wave propagation models. Computational and Mathematical Methods in Medicine 2012, 1-10 (2012).

3. Carson, J. \& van Loon, R. An implicit solver for $1 \mathrm{D}$ arterial network models. Int. J. Numer. Meth. Biomed. Engng. 02837, 1-16 (2016).

4. Ferranti, F., Tamburelli, V. \& Antonini, G. Rational macromodeling of 1 D blood flow in the human cardiovascular system. Int. J. Numer. Meth. Biomed. Engng. 1-17 (2015).

5. Keijsers, J., Leguy, C., Huberts, W., Narracott, A. \& Rittweger, J. A 1D pulse wave propagation model of the hemodynamics of calf muscle pump function. Int. J. Numer. Meth. Biomed. Engng. 1-20 (2015).

6. Wang, L.-J. J., Yao, Y., Feng, L.-S. S. et al. Noninvasive and quantitative intracranial pressure estimation using ultrasonographic measurement of optic nerve sheath diameter. eng. Scientific Reports 7, 1-7 (2017).

7. Huberts, W., Bode, A., Kroon, W. et al. A pulse wave propagation model to support decision-making in vascular access planning in the clinic. Medical Engineering and Physics (2011).

8. Marsden, A. Simulation based planning of surgical interventions in pediatric cardiology. Physics of Fluids 25, 1-20 (2013).

9. Bessems, D., Rutten, M. \& van de Vosse, F. A wave propagation model of blood flow in large vessels using an approximate velocity profile function. J. Fluid Mech 580, 145-168 (2007).

10. Perdikaris, P., Grinberg, L. \& Karniadakis, G. An effective fractal-tree closure model for simulating blood flow in large arterial networks. Annals of Biomedical Engineering 43, 1432-1442 (2014).

11. Van de Vosse, F. \& Stergiopulos, N. Pulse Wave Propagation in the Arterial Tree. Annual Review of Fluid Mechanics 43, 467-499 (2011).

12. Thomas, C., Nithiarasu, P. \& Bevan, R. The locally conservative Galerkin (LCG) method for solving the incompressible Navier Stokes equations. International Journal for Numerical Methods in Fluids 57, 1771-1792 (2008).

13. Alastruey, J., Parker, K. \& Sherwin, S. Arterial pulse wave haemodynamics 401-443 (2012).

14. Watanabe, S., Blanco, P. \& Raúl, F. Mathematical model of blood flow in an anatomically detailed arterial network of the arm. ESAIM: Mathematical Modelling and Numerical Analysis 47, 961-985 (2013).

15. Müller, L., Parés, C. \& Eleuterio, F. Well-balanced high-order numerical schemes for one-dimensional blood flow in vessels with varying mechanical properties. Journal of Computational Physics 242, 53-85 (2013).

16. Anderson, J. Computational Fluid Dynamics: The Basics with Applications (McGraw-Hill, 1995).

17. Felipa, C. A. Introduction to Finite Element Methods: Chapter 9 Multifreedom Constraints II (2004).

18. Hughes, T. \& Lubliner, J. On the one-dimensional theory of blood flow in the larger vessels. Mathematical Biosciences 18, 161-170 (1973).

19. Sherwin, S. J., Franke, V., Peiró, J. \& Parker, K. One-dimensional modelling of a vascular network in space-time variables. Journal of Engineering Mathematics 47, 217-250 (2003). 


\section{2 | CHAPTER 5}

20. Formaggia, L., Lamponi, D. \& Quarteroni, A. One-dimensional models for blood flow in arteries. Journal of Engineering Mathematics 47, 251-276 (2003).

21. Murgo, J., Westerhof, N., Giolma, J. \& Altobelli, S. Aortic Input Impedance in Normal Man : Relationship to Pressure Wave Forms. Circulation 62, 105-116 (1980).

22. Westerhof, N., Elzinga, G. \& Sipkema, P. An artificial arterial system for pumping hearts. Journal of applied physiology 31, 776-81 (1971).

23. Qureshi, M., Vaughan, G., Sainsbury, C., Johnson, M., Peskin, C. \& Olufsen, M. Numerical simulation of blood flow and pressure drop in the pulmonary arterial and venous circulation. Biomech Model Mechanobiol. 1137-1154 (2014).

24. Mynard, J. \& Valen-sendstad, K. A unified method for estimating pressure losses at vascular junctions. Int. J. Numer. Meth. Biomed. Engng. 1-23 (2015).

25. Bessems, D., Giannopapa, C. G., Rutten, M. C. M. \& van de Vosse, F. N. Experimental validation of a time-domain-based wave propagation model of blood flow in viscoelastic vessels. Journal of Biomechanics 41, 284-291 (2008).

26. Ghigo, A., Lagrée, P. \& Fullana, J. A time-dependent non-Newtonian extension of a $1 \mathrm{D}$ blood flow model. J Nonnewton Fluid Mech. 36-49 (2018).

27. Reymond, P., Merenda, F., Perren, F. et al. Validation of a one-dimensional model of the systemic arterial tree. American journal of physiology. Heart and circulatory physiology 297, H208-22 (2009). 


CHAPTER

General Discussion 


\subsection{Motivation and aim}

P

E R F O R M IN G measurements of the brain is crucial for the diagnosis of cerebral pathologies and the assessment of injury severity $[1,2]$. Moreover, continuous monitoring allows clinicians to evaluate the effect of their treatment strategies[2]. Unfortunately, most existing methods are either invasive or limited by the restricted accessibility of the brain. Some promising non-invasive methods for cerebral monitoring have been developed but they cannot yet match the current gold standards used for monitoring and decision-making and, hence, need improvement. In this thesis we aimed to improve the non-invasive assessment of two cerebral pathologies by developing two new technologies.

In the first part, our goal was to evaluate the current optic nerve sheath diameter (ONSD) assessment methodologies as well as to develop an algorithm for automatic estimation of the ONSD. The latter should eliminate the large inter- and intraobserver variabilities that are present in current manual assessment approaches and that hamper the ONSD as a non-invasive surrogate marker for the detection of elevated intracranial pressure (ICP).

In the second part, our objective was to improve the estimation of boundary conditions (BCs) for 3D hemodynamic aneurysm rupture risk simulations using a 1D pulse wave propagation model (PWPM). Such hemodynamic rupture risk models can assist in clinical decision-making but are dependent on proper boundary conditions. However, BCs for these simulations can often not be measured in clinical practice and using PWPMs to estimate them is currently the best available alternative $[3,4]$. We evaluated which aspects of the PWPM affect the estimation of the BCs and evaluated how these aspects influenced the corresponding $3 \mathrm{D}$ model-based rupture risk indices.

\subsection{Part I: Non-invasive ICP assessment using the ONSD}

\subsubsection{Major findings and clinical considerations}

Our literature review in Chapter 2 revealed poor reporting of, and large differences in ONSD assessment methodologies. Especially variations in placement of the ultrasound markers used to delineate the optic nerve sheath were found, which resulted in differences in ONSD values that could be as large as $1.2 \mathrm{~mm}$. Furthermore, we observed that the various methods had different sensitivities to changes in ICP resulting in discrepancies in ONSD values up to $0.6 \mathrm{~mm}$. These inconsistencies in ONSD assessment methodologies hamper the full potential of the ONSD as a noninvasive surrogate marker for elevated ICP $[5,6]$ because they result in differences in ONSD values that are of the same order of magnitude as the difference in ONSD 
values between patients with normal and elevated ICP as shown in Chapter 2 and literature[7-9]. In Chapter 2, we therefore composed a set of recommendations for ONSD assessment as a first step to decrease the observer variability in measured ONSD values caused by differences in ultrasound marker placement. However, the true impact of these recommendations on the reduction in ONSD variability depends on operator compliance.

In Chapter 3 we developed an automated algorithm that was able to automatically determine ONSD values from B-mode ultrasound images. The ONSD values obtained with the algorithm were comparable to ONSD values determined by medical experts but had no observer variability because the algorithm requires no user-input. Moreover, the mean differences in ONSD values between the algorithm and the experts were found to be approximately $0.1 \mathrm{~mm}$ and thus significantly smaller than the $1 \mathrm{~mm}$ difference observed between patients with normal and elevated ICP. In summary, we developed an algorithm that is clinically accurate and comparable to another fully automatic ONSD algorithm[10]. However, in contrast to the existing algorithm, our algorithm is also applicable to images without a clear distinction between the retrobulbar fat and the hyperechoic bands and to images that do not have hyperechoic bands at all. Our algorithm therefore provides a new solution to eliminate variabilities in ONSD values caused by manual assessment of the ONSD and can be used to obtain more accurate ONSD threshold values in future clinical studies.

Despite the fact that our algorithm solves the problem of discrepancies in ONSD values due to manual ONSD estimation, there are more aspects that can compromise the accuracy of the cut-off values for ICP estimation and that have to be investigated, i.e. the quality and characteristic appearances of the ultrasound images, and the ICP measurements. Ultrasound images do not only depend on the occurrence of image artefacts or physiological aspects of the patient that is imaged, but also on the skill and experience of the operator. Moreover, differences in probe placement or even incorrect imaging of the optic nerve can result in an altered depiction of the optic nerve and its sheath, leading to inconsistencies in the corresponding ONSD assessment. It is therefore crucial that the image acquisition is standardized. Inaccuracies within the invasive ICP measurements due to drift, improper calibration, or incorrect placement of the measurement devices will also affect the assessment of accurate ONSD threshold-values. It is therefore also important to improve the ICP measurements.

In order to obtain more consistent ONSD cut-off values and to make automated ONSD algorithms more widely applicable in the clinical settings, it is important to investigate to what extend the variabilities in optic nerve sonography and ICP measurements can be improved by the development of standardized measurement protocols, before we further improve the automated assessment of the optic nerve sheath diameter. 


\subsubsection{Limitations and Future work}

The automated segmentation algorithm we developed has some limitations. First of all, the segmentation is performed offline instead of on the ultrasound machine itself, which might hold back the deployment of the automatic segmentation in standard clinical practice. It is therefore important to incorporate our algorithm in the software of ultrasound scanners to increase the feasibility of the algorithm in the clinical setting.

Moreover, our algorithm has problems handling poor quality images, whereas the experts are able to extrapolate the sheath outline over large regions in case of a non-obvious distinction between retrobulbar fat and hyperechoic bands. However, it remains an open question how accurate such (manual) extrapolations are because our results showed differences in ONSD estimations between the two observers. In order to develop segmentation algorithms that match the "extrapolation and segmentation capabilities" of the human brain, the "segmentation rules" of the brain, as developed through evolution, need to be mimicked. However, identification of these advanced "rules" is not easy and implementing all these rules in an algorithm might be even harder. Machine-learning algorithms might be useful here[11] as they do not require a full definition of the "extrapolation-rules". By feeding machine-learning algorithms with annotated data, in our case B-mode ultrasound images where the delineation of the optic nerve sheath is already marked by experts, one can let the computer figure out its own "rules" that are needed to match the expert segmentation. However, also machine-learning has some drawbacks. First of all, a machine-learning algorithm is only as good as the data it is trained with. Therefore, a large amount of good quality ultrasound images is needed to train the machine-learning algorithm. These images should be representative of the ultrasound images that are encountered within the field of optic nerve sheath sonography and there should be consensus about the "true" annotation of the optic nerve sheath within these images. Second of all, we have no idea which image features the computer utilizes in order to segment the outline of the optic nerve sheath because the computer makes up its own rules behind the scenes. This makes it more difficult to "change" the rules of the machine-learning if the algorithm needs to be adapted to images with different characteristics. Nevertheless, whether we want to reduce the variability in ONSD measurements by either improving the accuracy of manual segmentations, of automated segmentation algorithms, or of machine-learning segmentation algorithms, it is crucial to improve the quality and consistency of the ultrasound images.

Another limitation of our work is that we did not validate whether the automaticallyderived ONSD values correlate better with invasively measured ICP than the manually-derived ONSD values and whether the algorithm indeed improves the accuracy of ONSD as a non-invasive surrogate marker for elevated ICP. As men- 
tioned earlier, the assessment of accurate ONSD cut-off values for the detection of elevated ICP depends on the uncertainties within the ONSD and ICP values. If the reduction in the ONSD variability by means of automatic assessment does not prove to be sufficient for accurate ONSD cut-off values, we should focus on reducing the discrepancies in optic nerve sonography and ICP measurements.

But even if we manage to reduce all the measurements variabilities, it remains to be seen if the ONSD can, without a doubt, be used as a non-invasive surrogate marker for patient-specific ICP predictions and replace the current invasive ICP monitoring. Humans come in different shapes and sizes and the diameter of the optic nerve and the surrounding sheath are most likely also subject to inter-subject variability. This inter-subject variability will subvert the discriminative power of the ONSD, especially in borderline cases. As a consequence, it might well be that the ONSD on its own cannot be used to estimate the exact ICP of a patient but can only be used to monitor increasing or decreasing ICP trends within a patient. To correct for the effect of inter-subject variability and to increase the discriminative power of the ONSD it might therefore be useful to combine the current ONSD assessment with other related metrics such as optic nerve sheath pulsatility[12] or the eyeball transverse diameter[13-16].

\subsection{Part II: Estimation of cerebral boundary conditions}

\subsubsection{Major findings and clinical considerations}

Hemodynamic aneurysm models can aid in the clinical decision-making because they can provide hemodynamic indices $[17,18]$ that give additional information about the aneurysm rupture risk compared to morphological metrics alone [17, 19]. However, simulating accurate rupture risk is only possible when patient-specific boundary conditions (BCs) are available[3, 17, 20-22]. In Chapter 4 we showed that a pulse wave propagation model (PWPM) might be a suitable approach to estimate $\mathrm{BCs}$ for $3 \mathrm{D}$ aneurysm rupture risk models when no patient-specific measurements are available, a finding that is in accordance with $[3,4]$. However, one should always consider that the BCs are approximations and are thus affected by model uncertainties that will propagate into the uncertainty of the rupture risk indices. We showed that population-based variations in macro-vascular properties of the PWPM can change the $\mathrm{BCs}$ and corresponding rupture risk indices with an order of magnitude similar to differences between ruptured and unruptured aneurysms. It is therefore crucial to adapt the PWPM to patient-specific conditions.

However, making the whole PWPM patient-specific is practically impossible. Luckily, it is not necessary to set all model parameters to patient-specifically measured values because not all model parameters have the same effect on the boundary 
conditions. Using our uncertainty quantification (UQ) and sensitivity analysis (SA) approach it is not only possible to quantify the uncertainty in outputs of interest resulting from the uncertainty in each model parameter, but also to identify model parameters that contribute significantly to the variation in the output metrics, not just on their own but also in combination with other parameters (interactions). Identification of such influential parameters tells us which variables should be measured patient-specifically and which might be set to population-based values. This enables us to create a patient-specific PWPM that can assess the patient-specific $\mathrm{BC}$ needed for personalized rupture risk assessment. Moreover, it allows clinicians and researchers to focus on measuring these parameters in clinic or finding new and better ways to measure these parameters if needed.

However, the estimation of accurate patient-specific BCs using our PWPM does not only depend on the accuracy of model input parameters but also on model assumptions. Model assumptions determine how well the model can describe the true underlying physiological mechanisms, known as model discrepancy. Similarly as with the variations in 1D model parameters, model assumptions can influence pressure and flow BCs. In Chapter 5 we investigated one model assumption that is debated in literature, i.e. the static pressure-coupling at bifurcations[23]. Changing our pressure-coupling increased the systolic pressure in the proximal arteries of our model by $1 \%$ and decreased the systolic pressures in the distal arteries by 3 $\%$. Although these findings indicate that the BCs obtained from our 1D model are hardly having any bias due to our pressure-coupling assumption, it might be possible that the effect becomes significant when we adjust our model to match the physiological envelop of a patient in order to simulate patient-specific BCs. Nevertheless, using our UQ \& SA approach we can also identify important model assumptions and use this information to personalize our PWPM and the simulated BCs even further.

Although we have developed all the tools needed to personalize our PWPM, our framework cannot yet be used to perform patient-specific simulations within the clinical setting because we still need to determine which model parameters and assumptions are crucial for the assessment of patient-specific BCs and personalized aneurysm rupture risk indices, and because we did not yet validate if our framework is feasible within the clinical setting.

In order to bring computer models into the clinic, the optimal trade-off between the model's complexity and the (clinical) data required to calibrate (personalize) the model has to be found. Sometimes the parameters needed for the calibration can simply not be measured in patients due to technological or ethical limitations, other times the measurements are just not feasible within the clinical work flow. As a result, model assumptions have to be made which can lead to model discrepancies and thus to model output errors that can prevent the estimation of accurate 
diagnostic metrics. However, it is also possible that the required measurements are already part of the standard patient care but that the quality of the available clinical data is not good enough for model calibration. In the clinical setting it is often no problem when measurements contain a measurement error because data are frequently only used to monitor trends within a patient. However, these errors can be disastrous for the correct modeling of physiological mechanisms.

Besides the trade-off between model complexity and model input data, it is also important that the simulated model output metrics are clinically useful. Computer models can be used to calculate a wide variety of metrics but these will only benefit clinical decision-making if they have an added value for diagnosing or monitoring patients.

In order to adjust computational models and the quality and availability of the clinical data to one another, an iterative and multidisciplinary[24] approach is needed to allow engineers and clinicians to learn from each other and to complement each other using their own specific knowledge. Only this way the full potential of our model (and models in general) can be brought into the clinical setting and thereby benefit clinicians and patients due to their diagnostic abilities.

\subsubsection{Limitations and Future work}

The outcome of the uncertainty quantification depends on a correct definition of the input uncertainty ranges, something that can be difficult to define. We based the uncertainty of our 1D PWPM parameters on physiological population ranges found in literature. In addition, we incorporated a physiological relation between some parameters using age. Nevertheless, it is possible that we carried out simulations with non-physiological combinations of parameters. This could potentially lead to parameter contributions that deviate from the truth giving us an incorrect idea of the importance of parameters. However, by comparing the model response, e.g. systemic pressures or reflections at bifurcations, with known physiological values, the non-physiological simulations could be detected[25]. Non-physiologically combinations can also occur when we set certain model parameters to patient-specific values while keeping others at population-based values. To prevent incorrect risk predictions, it is important to detect such non-physiological combinations by analyzing the model responses.

Another limitation is the fact that we evaluated the influence of only a few model parameters and only one model assumption on our pressure and flow waveforms. Moreover, we did not take into account the model assumptions of the 3D rupture risk model and the uncertainties that might arise from the imaging and segmentation of the aneurysm geometry. The influence of these additional uncertainties could possibly be crucial for the simulation of personalized rupture risk indices 
and should therefore be investigated in future work. Despite the fact that we only investigated the influence and validity of a few model input uncertainties and model discrepancies, the pressure and flow waveforms of our virtual database showed good agreement with the simulated waveforms of Mulder et al. [26] and the physiological waveforms reported in Ford et al. [27]. Moreover, our 3D simulations showed results according to literature for both the simulated 3D flow patterns [3, 28, 29] as well as for the aneurysm rupture risk indices[17, 20, 30, 31].

As mentioned above, the development of models that can perform patient-specific predictions requires the correct choice of model input parameters and model assumptions. However, it is not always possible to include all the physiological processes that are needed for patient-specific simulations in a computer model, as some mechanisms are not completely understood and can therefore not be captured in physical equations. In such cases it might be necessary to resort to hybrid modeling techniques where the initial physiological simulation model is combined with other predictive methodologies, e.g. the unknown mechanism might be captured in a phenomenological model such as a multi-variate regression models.

It might be evident that more improvement steps are needed before our framework can be fully incorporated within the clinical settings. Though this requires a tremendous amount of work, these steps are necessary to develop patient-specific PWPMs that can assess the boundary conditions for 3D hemodynamic aneurysm rupture risk models to allow aneurysm rupture risk assessment in all cases where no measured patient-specific pressure and flow waveforms are available. However, this does not imply that PWPMs cannot be useful until we resolved all the above mentioned issues. Until then, PWPM can already be used to obtain new insights about disease or to find new metrics for the diagnosis of pathologies (see the next section).

\subsection{Technologies in the clinical setting}

In this thesis we demonstrated the application of technologies to improve existing non-invasive methods for the diagnosis and monitoring of cerebral pathologies. First of all, we showed the ability of image segmentation algorithms to segment ONSD from ultrasound B-mode images without any user input, hereby reducing the observer variability. While our algorithm showed great potential to segment ONSD from ultrasound B-mode images, it can also be used to segment the ONSD from Computed Tomography (CT) images. Performing CT scans when TBI patients are submitted to the hospital to assess the severity of the brain injury is standard clinical care in most hospitals. Extending the CT assessment with an automatic ONSD detection could give a first indication of the ICP while also having visual information about the state of the brain. 
However, we are not the only ones who successfully implemented an algorithm for the segmentation of medical images. Others have, for example, used automated algorithms for the segmentation of brain tumours[32] or carotid plaques[33]. In general, segmentation algorithms have the potential to increase the precision of manual segmentations, which could result in a better risk stratification or monitoring of patients. In addition, segmentation algorithms relieve clinicians from this time consuming task which leaves them with more time to practice other patient-related care. Moreover, automated analysis of medical images can be done on servers 24 hours a day, seven days a week. As a result more images can be processed, thereby allowing monitoring, diagnosis, and treatment planning of more patients.

In our work we also presented a framework in which PWPMs can be used to simulate patient-specific BCs for cerebral aneurysm rupture risk models. Besides the generation of BCs for other models, our PWPM model can also be used for risk stratification on its own as long as we can capture the underlying physiological mechanisms in our model. Researchers have already explored the feasibility of using PWPMs to assess the ICP[34, 35]. Although these models showed promising results, capturing the complete hemodynamic brain circulation including the cerebrospinal fluid and obtaining the correct parameter settings for patientspecific ICP predictions is not easy. To overcome this problem, the latest trend is data-augmentation [34, 36], a technique where model simulations and measurement data are combined in an advanced way in order to obtain better predictions. Wang et al. [34] showed how a PWPM in combination with cerebral blood flow and arterial blood pressure measurements, can be used to obtain better ICP predictions. Although computer models combined with data-augmention have shown good ICP predictions with an average error smaller than $5 \mathrm{mmHg}[34,36]$, the accuracy of the model predictions is often limited by the quality and consistency of the clinical measurement data.

Pulse wave propagation model can also be used to perform in silico trials to obtain insight into possible mechanisms behind certain pathologies or to find new metrics that could potentially be used to monitor/diagnose patients. By manipulation of model parameters or equations we can turn the "healthy" model into a "diseased" model. Using physiological ranges of healthy people and diseased patients the model input parameters can be adjusted and used to construct a virtual (patient) database. Comparison of the results obtained with the "healthy" and the "diseased" models with physiological changes observed in patients, allows us to develop new hypotheses about pathological mechanisms or to find new possible metrics that can then be further investigated in patients. In addition, we can investigate longitudinal changes by including adaptation/remodeling in our model. In a study of Melis et al. [37], small vessel disease was simulated by adjusting the radii and stiffnesses of the distal cerebral vessels in a PWPM. Next, changes in simulated waveforms of the 
proximal vessels were quantified by introducing new metrics that could possibly be used to diagnose patients with small vessel disease. The next step in their research would be to validate the discriminative power of these metrics by measuring them in small vessel disease patients and comparing them with values obtained in healthy controls.

Besides for monitoring or the diagnosis of diseases, PWPMs can also be used to support the surgeon with intervention planning. Treatment scenarios can be modeled and evaluated using patient data, thereby giving the surgeon advise on the possible best treatment option for the patient. An example is the surgical planning of vascular access in hemodialysis patients[38]. By feeding the PWPM with patient-specific flow and diameter data the PWPM model can predict the best location for the construction of the vascular access, hereby assisting the surgeon in his decision. We also illustrated how 3D-CFD models can be used to simulate complex flow patterns to derive rupture risk indices for cerebral aneurysms. However, CFD simulations are not limited to vascular applications such as the assessment of the risk of aneurysm rupture or aortic dissections[39]. They can, for example, also be used to simulate the air flow out of the lungs and assess the risk of the spread of infectious diseases to another person [40, 41], which is an application that is, at this moment, very topical and very important.

From our discussion above it is clear that technologies have great potential to improve clinical monitoring and decision-making when the effect of limitations, model assumptions, and model input uncertainties are taken into account. However, from the technologies developed in Part I and Part II it becomes clear that it is crucial to improve the quality of the clinical data that feed these technologies. In order for technologies and clinicians to better complement each other, it is important that engineers and clinicians cooperate and provide each other with continuous feedback. 


\subsection{Conclusion}

The monitoring and diagnosis of cerebral pathologies is crucial to prevent brain damage and even death in patients. Current monitoring options are limited and often invasive. Although non-invasive approaches are promising, they cannot yet match the current invasive gold standards.

In this thesis we aimed to use technologies to improve 1) the use of the optic nerve sheath diameter as a non-invasive assessment technique to assess intracranial pressure, and 2) the estimation of boundary conditions for cerebral aneurysm risk simulations using a pulse wave propagation model.

In the first part, we revealed inconsistencies in current ONSD assessment methodologies that hamper the full potential of the ONSD as a non-invasive surrogate marker for elevated ICP. To resolve the problem of inter- and intraobserver variabilities associated with manual ONSD assessment, we developed an automated algorithm to segment the ONSD from B-mode ultrasound images of sufficient quality. In addition, we proposed a set of guidelines to improve the quality of ultrasound image acquisition. These guidelines could be a first step towards the standardization of optic nerve sonography.

In the second part, we showed that 1D PWPMs can be used to simulate BCs for aneurysm rupture risk models but that model input uncertainty and model discrepancies affect the BCs and corresponding aneurysm rupture risk indices. Our technology provides the tools needed to take into account the effects of inputand model uncertainties and allows the construction of a patient-specific PWPM which can be used to estimate patient-specific BCs that are crucial for accurate hemodynamic aneurysm rupture risk prediction.

Based on this work it is evident that technologies can improve current non-invasive monitoring techniques. Nevertheless, more technological development and more accurate measurement data are needed before patients can fully benefit from such technologies. Hereto, a multidisciplinary approach is needed. 


\section{References}

1. Rangel-Castillo, L., Gopinath, S. \& Robertson, C. S. Management of Intracranial Hypertension. Neurologic Clinics 26, 521-541 (2008).

2. Galgano, M., Toshkezi, G., Qiu, X., Russell, T., Chin, L. \& Zhao, L. R. Traumatic brain injury: Current treatment strategies and future endeavors. Cell Transplantation 26, 1118-1130 (2017).

3. Marzo, A., Singh, P., Larrabide, I. et al. Computational hemodynamics in cerebral aneurysms: The effects of modeled versus measured boundary conditions. Annals of Biomedical Engineering 39, 884-896 (2011).

4. Mulder, G., Marzo, A., Bogaerds, A. C. B. et al. Patient-Specific Modeling of Cerebral Blood Flow: Geometrical Variations in a 1D Model. Cardiovascular Engineering and Technology 2, 334-348 (2011).

5. Bloria, S., Bloria, P. \& Luthra, A. Is it the time to standardize the procedure of ultrasound guided optic nerve sheath diameter measurement? Saudi Journal of Anaesthesia 13, 255 (2019).

6. Pichamuthu, K. K. \& Prithishkumar, I. J. Appearance of the Optic Nerve Sheath Diameter (ONSD) using Higher Frequency Linear Probes in Detection and Monitoring of Raised Intracranial Pressures-A Cadaveric Study. Journal of Clinical and Diagnostic Research, 10-13 (2019).

7. Lochner, P., Fassbender, K., Lesmeister, M. et al. Ocular ultrasound for monitoring pseudotumor cerebri syndrome. eng. Journal of neurology 265, 356-361 (2018).

8. Moretti, R., Pizzi, B., Cassini, F. \& Vivaldi, N. Reliability of optic nerve ultrasound for the evaluation of patients with spontaneous intracranial hemorrhage. eng. Neurocritical care 11, 406-410 (2009).

9. Lochner, P., Cantello, R., Fassbender, K. et al. Longitudinal Assessment of Transorbital Sonography , Visual Acuity , and Biomarkers for Inflammation and Axonal Injury in Optic Neuritis. 2017, 4-9 (2017).

10. Meiburger, K. M., Naldi, A., Michielli, N. et al. Automatic Optic Nerve Measurement: A New Tool to Standardize Optic Nerve Assessment in Ultrasound B-Mode Images. Ultrasound in Medicine and Biology 46, 1533-1544 (2020).

11. Cantisani, V., Grani, G., Tovoli, F., Piscaglia, F. \& Catalano, C. Artificial Intelligence: What is it and How can it expand the ultrasoun. Ultraschall in der Medizin (Stuttgart, Germany : 1980) 41, 356-360 (2020).

12. Padayachy, L., Brekken, R., Fieggen, G. \& Selbekk, T. Pulsatile dynamics of the optic nerve sheath and intracranial pressure: An exploratory in vivo investigation. Neurosurgery 79, 100-107 (2016).

13. Lee, D. H., Lee, S. H., Oh, J. H. et al. Optic nerve sheath diameter measured using early unenhanced brain computed tomography shows no correlation with neurological outcomes in patients undergoing targeted temperature management after cardiac arrest. Resuscitation 128, 144-150 (2018).

14. Kavi, T., Gupta, A., Hunter, K., Schreiber, C., Shaikh, H. \& Turtz, A. R. Optic Nerve Sheath Diameter Assessment in Patients with Intracranial Pressure Monitoring. Cureus 10 (2018).

15. Vaiman, M., Sigal, T., Kimiagar, I. \& Bekerman, I. Intracranial pressure assessment in traumatic head injury with hemorrhage via optic nerve sheath diameter. Journal of Neurotrauma 33, 2147-2153 (2016).

16. Vaiman, M., Gottlieb, P. \& Bekerman, I. Quantitative relations between the eyeball, the optic nerve, and the optic canal important for intracranial pressure monitoring. Head and Face Medicine 10, 1-6 (2014). 
17. Sarrami-Foroushani, A., Lassila, T., Gooya, A., Geers, A. J. \& Frangi, A. F. Uncertainty quantification of wall shear stress in intracranial aneurysms using a data-driven statistical model of systemic blood flow variability. Journal of Biomechanics 49, 3815-3823 (2016).

18. Boussel, L., Rayz, V., McCulloch, C. et al. Aneurysm growth occurs at region of low wall shear stress: Patient-specific correlation of hemodynamics and growth in a longitudinal study. Stroke 39, 2997-3002 (2008).

19. Xiang, J., Natarajan, S. K., Tremmel, M. et al. Hemodynamic-Morphologic Discriminants for Intracranial Aneurysm Rupture. Stroke 42, 144-152 (2011).

20. Karmonik, C., Yen, C., Grossman, R. G., Klucznik, R. \& Benndorf, G. Intra-aneurysmal flow patterns and wall shear stresses calculated with computational flow dynamics in an anterior communicating artery aneurysm depend on knowledge of patient-specific inflow rates. Acta Neurochirurgica 151, 479-485 (2009).

21. Spilker, R. L. \& Taylor, C. A. Tuning multidomain hemodynamic simulations to match physiological measurements. Annals of Biomedical Engineering 38, 2635-2648 (2010).

22. Pope, S. R., Ellwein, L. M., Zapata, C. L., Novak, V., Kelley, C. T. \& Olufsen, M. S. Estimation and identification of parameters in a lumped cerebrovascular model. Mathematical Biosciences and Engineering 6, 93-115 (2009).

23. Azer, K. \& Peskin, C. S. A one-dimensional model of blood flow in arteries with friction and convection based on the Womersley velocity profile. Cardiovascular Engineering 7, 51-73 (2007).

24. Huberts, W., Heinen, S. G., Zonnebeld, N. et al. What is needed to make cardiovascular models suitable for clinical decision support? A viewpoint paper. Journal of Computational Science 24, 68-84 (2018).

25. Willemet, M., Chowienczyk, P. \& Alastruey, J. A database of virtual healthy subjects to assess the accuracy of foot-to-foot pulse wave velocities for estimation of aortic stiffness. American Journal of Physiology - Heart and Circulatory Physiology 309, H663-H675 (2015).

26. Mulder, G., Bogaerds, A. C. B., Rongen, P. \& van de Vosse, F. N. The influence of contrast agent injection on physiological flow in the circle of Willis. Medical Engineering and Physics 33, 195-203 (2011).

27. Ford, M. D., Alperin, N., Lee, S. H., Holdsworth, D. W. \& Steinman, D. A. Characterization of volumetric flow rate waveforms in the normal internal carotid and vertebral arteries. Physiological Measurement 26, 477-488 (2005).

28. Cebral, J. R., Sheridan, M. \& Putman, C. M. Hemodynamics Before and After Bleb Formation in Cerebral Aneurysms. American Journal of Neuroradiology 31, 304-310 (2010).

29. Cebral, J. R., Castro, M. A., Burgess, J. E., Pergolizzi, R. S., Sheridan, M. J. \& Putman, C. M. Characterization of cerebral aneurysms for assessing risk of rupture by using patient-specific computational hemodynamics models. AJNR. American journal of neuroradiology 26, 2550-9 (2008).

30. Jansen, I. G. H., Schneiders, J. J., Potters, W. V. et al. Generalized versus Patient-Specific Inflow Boundary Conditions in Computational Fluid Dynamics Simulations of Cerebral Aneurysmal Hemodynamics. Americal Journal of NeuroRadiology 35, 1543-1548 (2014).

31. Karmonik, C., Yen, C., Diaz, O., Klucznik, R., Grossman, R. G. \& Benndorf, G. Temporal variations of wall shear stress parameters in intracranial aneurysms: importance of patient-specific inflow waveforms for CFD calculations. Acta neurochirurgica 152, 1391-1398 (2010).

32. Mascarenhas, L. R., Ribeiro, A. d. S. \& Ramos, R. P. Automatic segmentation of brain tumors in magnetic resonance imaging. Einstein (São Paulo) 18, 1-8 (2020). 
33. Cheng, J., Li, H., Xiao, F. et al. Fully Automatic Plaque Segmentation in 3-D Carotid Ultrasound Images. Ultrasound in Medicine and Biology 39, 2431-2446 (2013).

34. Wang, J.-X., Hu, X. \& Shadden, S. C. Data-augmented modeling of intracranial pressure, 1-26 (2018).

35. Ryu, J., Hu, X. \& Shadden, S. C. A Coupled lumped-parameter and distributed network model for cerebral pulse-wave hemodynamics. Journal of Biomechanical Engineering 137 (2015).

36. Imaduddin, S. M., Fanelli, A., Vonberg, F. W., Tasker, R. C. \& Heldt, T. Pseudo-bayesian modelbased noninvasive intracranial pressure estimation and tracking. IEEE Transactions on Biomedical Engineering 67, 1604-1615 (2020).

37. Melis, A., Moura, F., Larrabide, I. et al. Improved biomechanical metrics of cerebral vasospasm identified via sensitivity analysis of a $\mathrm{D}$ cerebral circulation model. Journal of Biomechanics 9o, 24-32 (2019).

38. Caroli, A., Manini, S., Antiga, L. et al. Validation of a patient-specific hemodynamic computational model for surgical planning of vascular access in hemodialysis patients. Kidney International 84, 1237-1245 (2013).

39. Wee, I., Ong, C. W., Syn, N. \& Choong, A. Computational Fluid Dynamics and Aortic Dissections: Panacea or Panic? Vascular and Endovascular Review 1, 27 (2018).

40. Vuorinen, V., Aarnio, M., Alava, M. et al. Modelling aerosol transport and virus exposure with numerical simulations in relation to SARS-CoV-2 transmission by inhalation indoors. Safety Science 130, 104866 (2020).

41. Gao, N. \& Niu, J. Transient CFD simulation of the respiration process and inter-person exposure assessment. Building and Environment 41, 1214-1222 (2006). 


Appendices 

Valorization 


\subsection{Introduction}

E R E B RO vascular pathologies can have a major impact on a patient's quality of life or even result in the death of the patient. Therefore, early diagnosis and adequate monitoring of cerebral diseases is very important. However, performing measurements on the brain is challenging because the accessibility of the brain is limited by the skull and opening the skull is very invasive. To obtain crucial information about the status of the brain and the pathology, new and preferably non-invasive measurement techniques are being developed. Although new non-invasive techniques show promising results, these techniques are often not good enough to be deployed within the clinical setting and, hence, need to be improved. Moreover, risk stratification of some cerebral diseases can not be performed because the associated metrics can not yet be measured. In these cases it might be possible to estimate the diagnostic metrics by using information measured at other locations within the body. Technological advances, such as computer models, can potentially play an important role in the improvement and development of the non-invasive monitoring and assessment methods of the future. In this thesis we showed how technologies can be used to improve the non-invasive assessment of intracranial pressure in patients suffering from traumatic brain injury (Chapters 2 and 3) or to improve the non-invasive assessment of aneurysm rupture risk in patients suffering from cerebral aneurysms (Chapters 4 and 5 ).

\subsection{Clinical and Societal relevance}

In Part I we aimed to improve the assessment of the optic nerve sheath diameter (ONSD) for the detection of elevated intracranial pressure (ICP). The work performed in Chapter 2 highlights the influence of measurement inaccuracies on ONSD values which includes the sensitivity of these ONSD values to changes in ICP. Our work makes clinicians (and researchers) aware of the possible intra- and interobserver variabilities present within manual ONSD measurements as well as their corresponding influence on risk stratification. By exposing the discrepancies in manual assessment methods that cause the uncertainties in ONSD threshold values, we show the importance of standardization by methodological guidelines and the need to comply to such guidelines. Our set of ONSD assessment guidelines proposed in Chapter 2 is a first step towards standardization of ONSD assessment. Only by standardized assessment methods we can ensure good quality measurements and achieve accurate discriminative clinical metrics, not only for pathologies and applications discussed in this thesis, but for clinical monitoring and diagnosing applications in general. 
The completely automated ONSD algorithm, presented in Chapter 3, does not only relieve clinicians from the task of manually assessing the ONSD, but also improves the accuracy of ONSD assessment as it overcomes the intra- and interobserver variability associated with manual ONSD assessment. Our algorithm can easily be applied within the clinical settings because it is applicable to B-mode ultrasound images that are already regularly used to image the optic nerve sheath and because our algorithm requires no additional knowledge or effort from clinicians. In addition, our presented technology can be incorporated fairly easy within the clinical setting by implementing our algorithm on ultrasound scanners. Moreover, our algorithm will be published in journals associated with the field making it widely available and, hence, increasing the number of patients that can benefit from our work even further. Although further research should be performed to validate if the automatic ONSD assessment indeed results in more accurate ONSD cut-off values for the detection of patients with elevated ICP, such a non-invasive ICP assessment brings multiple benefits for both society and patients. First of all, the physiological burden on these already critically-ill traumatic brain injury patients is greatly reduced because the very invasive ICP sensors become redundant. In addition, the hospitalization cost of these patients might be reduced as patients can be treated more effectively. Moreover, non-invasive methods allow ICP monitoring in non-comatose patients normally not subjected to ICP measurements because of its invasive nature, e.g. children with hydrocephalus, also improving clinical care for such patient groups.

In Part II we explored the feasibility of a pulse wave propagation model (PWPM) for the estimation of patient-specific boundary conditions for cerebral aneurysm rupture risk models. Most clinical treatments are based on their effectiveness within patient populations. However, these treatments might not be as effective for every individual patient within this population. Individualized treatments would be the pinnacle of clinical practice but require assessment of the multifactorial physiological status of every individual patient. Unfortunately, current measurement methods are not capable of measuring and taking into account all the relevant factors needed for an accurate and detailed assessment of the patient's status. Moreover, even with improved measurement techniques it might still not be possible to directly measure every relevant parameter/process within the human body. In such cases it might be possible to estimate these metrics by using information from other locations within the body. Our PWPM that mimics physiological processes of the human body by combining physical laws and physiological knowledge can be used to simulate these unmeasurable variables using patient data that can be measured at other locations in the human body. This allows for the diagnosis and monitoring of cerebral pathologies that would otherwise not be possible. Moreover, these models can also be used to simulate the effect of certain treatment 
options[1] and any possible changes within the patients, hereby aiding in the clinical decision-making. Finally, such computer models might be used to link many of these aspects together, hereby creating a more complete picture of the physiological state of the patient, which allows for a more patient-specific treatment.

In order to simulate patient-specific diagnostic metrics it is important that computer models describe the relevant patient characteristics as closely as possible. Unfortunately, not all model settings can be measured patient-specifically due to for example, technical or ethical limitations. In addition, not all the processes within the human body can be captured within physical equations because some processes will never be completely understood. Therefore, researchers need to deal with the fact that some model parameters have to be set to population-based values and that some processes have to be simplified using model assumptions. Using the framework presented in Chapters $\mathbf{4}$ and $\mathbf{5}$ one can determine the uncertainties within the simulation outputs resulting from the errors present in the model input parameters. Moreover, it enables one to pinpoint those model parameters and model assumptions that have a large influence on the model outputs and should therefore resemble the true patient-specific situation as closely as possible. Identification of such important parameters allows researchers to focus their efforts on measuring these variables more accurately or on developing new technologies that can be used to perform accurate measurements of the necessary parameters within patients. Therefore, our framework contributes to the efficient development of patient-specific models.

Although frameworks for patient-specific aneurysm rupture risk have already been developed $[2,3]$, complete frameworks that can capture the total physiological state of a patient and assess patient-specific treatment methods are still far from bedside application. In order to incorporate such frameworks into daily clinical practice, a multi-disciplinary collaboration between engineers and clinicians is needed. Hereto, it is important that both clinicians and researchers are aware of the existence and possibilities of the tools and computer models as described in this thesis. The methods presented in this thesis are or will be published in scientific journals and are therefore available for other investigators who are interested in creating model-based frameworks that can support patient-specific decision-making. Hopefully, our work contributes to a future where patient-specific treatment is daily clinical practice. 


\section{References}

1. Caroli, A., Manini, S., Antiga, L. et al. Validation of a patient-specific hemodynamic computational model for surgical planning of vascular access in hemodialysis patients. Kidney International 84, 1237-1245 (2013).

2. Cebral, J. R., Castro, M. a., Appanaboyina, S., Putman, C. M., Millan, D. \& Frangi, A. F. Efficient pipeline for image-based patient-specific analysis of cerebral aneurysm hemodynamics: Technique and sensitivity. IEEE Transactions on Medical Imaging 24, 457-467 (2005).

3. Detmer, F. J., Chung, B. J., Mut, F. et al. Development of a statistical model for discrimination of rupture status in posterior communicating artery aneurysms. Acta neurochirurgica (2018). 

Acknowledgements

(Dankwoord) 
$\mathrm{H}$

IE R is het dan, het hoofdstuk waar de meeste mensen die dit boekje openslaan direct naar op zoek gaan: het dankwoord. Net als zo veel anderen, had óók ik mijn $\mathrm{PhD}$ thesis niet tot een goed einde kunnen brengen zonder de hulp van een hele hoop mensen. Op de eerste plaats wil ik mijn promotoren en copromotor bedanken voor hun bijdrage aan mijn $\mathrm{PhD}$ en aan dit proefschrift.

Beste Tammo, ondanks dat onze samenwerking niet altijd even soepel verliep heeft jouw kritische blik de kwaliteit van mijn werk naar een hoger niveau getild. Alhoewel jouw met vulpen geschreven commentaar vaak moeilijk te ontcijferen was, zorgde zowel het inhoudelijke als het tekstuele commentaar ervoor dat de stukken (na meerdere revisies) uiteindelijk beter leesbaar en beter opgebouwd waren. Ook jouw bijna onmenselijke oog voor detail heeft veel van mijn plaatjes omgetoverd tot een waar kunstwerk. En na het verhaal over de man die van zijn vrouw wilde scheiden vanwege ontbrekende eenheden bij grafiekassen, kon ik ook dit niet meer fout doen.

Beste Wouter, in 2015 begon ik bij jou aan mijn afstudeerproject. Ik ken je nu dus al heel wat jaren en heb inmiddels geleerd dat je dit stukje tekst interessanter zou vinden en makkelijker zou kunnen begrijpen als het in formuletaal geschreven was. Ondanks de talloze keren dat we samen hebben gezeten om aan een nieuw raadsel van formules te werken, zal ik jouw kwaliteit van het omgaan met formules nooit kunnen evenaren. Minder blij werd ik meestal van je zeer enthousiaste commentaar. Met je favoriete spreuk "het lijkt meer dan dat het is", probeerde je me dan weer altijd gerust te stellen en uit te leggen wat er veranderd moest worden. Maar ook als ik andere vragen had of ergens mee zat, kon ik altijd bij je binnenlopen of met je bellen, zelfs in de late uurtjes of tijdens je vakanties. Soms vraag ik me af hoeveel vakantieuren je hierdoor eigenlijk bent misgelopen. Bedankt voor je hulp!

Beste Werner, jouw kennis op het gebied van de anatomie en het functioneren van de hersenen zijn zeer nuttig geweest bij het uitvoeren van mijn werk. Jij zorgde niet alleen dat het onderzoek aansloot op de klinische vraag, maar hielp me ook bij het zelf uitvoeren van echometingen. Ik stond vaak versteld van jouw interesse in nieuwe technologieën die mogelijk gebruikt konden worden voor klinisch onderzoek. Dit motiveerde mij om de literatuur in te duiken en mijn kennis over het brein verder uit te breiden.

Beste Erik, ondanks dat je niet vermeld staat als lid van het promotieteam, heb jij ook een heel grote rol gespeeld tijdens mijn promotietraject. Niet alleen gaf je nuttige input tijdens de tweewekelijkse meetings, maar hielp je me ook met het opzetten van diverse meetopstellingen wanneer ik weer een experiment of meting wilde uitvoeren. Daarnaast hielp je me telkens wanneer ik bij jou naar binnenliep 
met de mededeling dat de opstelling niet werkte vanwege een kapot kabeltje. Jij nam dan de tijd om me uit te leggen waarom voor deze nieuwe opstelling weer een ander kabeltje nodig was en verwees mij door naar de juiste persoon. Mijn dank hiervoor.

Prof. Temel, dr. Bonizzi, Prof. van der Horst, Prof. Mischi en dr. Tromp, bedankt dat jullie als leescommissie mijn proefschrift op zo'n korte tijd hebben beoordeeld.

Lieve Jeanette, jouw enthousiasme voor het werken in de kliniek is ongekend. Jij wist me dan ook bijna altijd te motiveren als het tegenzat. Jouw ervaring en goede connecties met het zorgpersoneel van de IC maakten het voor mij makkelijker om ondanks mijn gebrek aan ervaring op de IC toch mijn weg te vinden. Je belde mij elke keer (weekend of niet) als er weer een nieuwe patiënt was en regelde dan met het zorgpersoneel dat je samen met "je vriend" de metingen kon doen. Zonder jouw hulp was het uitvoeren van de patiëntmetingen zeker niet zo goed verlopen. Marcel, de uren die we daadwerkelijk met elkaar doorbrachten zijn redelijk beperkt gebleven. Toch deed jij er achter de schermen alles aan om ervoor te zorgen dat ik mijn metingen op de IC kon doen. Steeds weer wist jij het IC-personeel te overtuigen van de noodzaak van deze metingen, en je instrueerde hen waar nodig om net even iets meer aandacht te besteden aan zaken die de kwaliteit van onze data ten goede zouden komen. Nanon, ik leerde je natuurlijk kennen als kantoorgenoot van Jeanette. Je hielp me graag met het stangen van Jeanette als die toch al ietwat gestrest was. Blijf dit vooral doen! KNF-laboranten, bedankt voor jullie ondersteuning bij mijn onderzoek op de KNF.

Tim, Juliana, Yves en Stanley, het begeleiden van jullie afstudeerprojecten heeft mij veel plezier opgeleverd. Alhoewel er vaak afgeweken moest worden van het aanvankelijke idee, heeft het interessante discussies en inzichten opgeleverd. Tim, onze samenwerking heeft zelfs een hoofdstuk voor deze thesis opgeleverd. Stan, het project waar ik jou bij ondersteunde (of moet ik zeggen: waar jij mij bij ondersteunde), heeft gezorgd voor een versnelling in het afronden van het automatische ONSD algoritme, dankjewel.

Beste (ex-) collega's van BME, bedankt voor de vriendelijke en relaxte sfeer die altijd op het lab aanwezig was. Erik, ondanks dat het met de huidige maatregelen niet meer mag, vond ik het fijn om samen met jou een cubicle te delen. Het wederzijds afkraken van de plaatjes op elkaars scherm, zorgde zo nu en dan toch voor wat ontspanning. Daarnaast zag mijn bureau er altijd keurig opgeruimd uit in vergelijking met dat van jou. Frank, als creatieve beheerder van de (kerst-/paas-/konings-/etc.) boom heb ik genoten van de ideeën die je had om onze boom nog een maandje 
langer te laten staan. Bennis, jouw muzikale inbreng in de "togethertube" in combinatie met je kenmerkende lach hebben veel vrijdagmiddagen opgefleurd. Ben, ondanks dat ik het idee had dat stressballetjes vaak mijn kant uit kwamen, kon ik bij jou altijd terecht voor advies over auto's, het kopen van een huis of natuurlijk over het spelen van Counter Strike. Myrthe, ondanks dat jij pas later aansloot bij onze afdeling, mengde jij je meteen goed in de groep. Ik kon je altijd vragen om commentaar op mijn poster of plaatjes. Het plan om beneden koffie of chocomel te halen, dat altijd "spontaan" ontstond als je weer even kwam buurten, kon ik ook nooit afslaan, but that's something else. Letty, ook jij sloot pas wat later tijdens mijn $\mathrm{PhD}$ aan in ons lab. Toch was je altijd in voor een praatje of voor leuke verhalen over je tripjes naar België in het weekend. Tijmen, zorg goed voor "Kuuschke". Nick, succes met boulderen. Tim, bedankt voor "Die Mayrhofner". Anneloes, als een van de laatste nieuwe PhD-studenten in het lab is het aan jou, Myrthe en Letty om het lab gezellig te houden. Niek, het beeld van jou in je met kerstlampjes verlichte kamer terwijl je keihard Mariah Carey meezingt, zal ik nooit vergeten. Pamir, bij jou kon ik altijd even binnenlopen voor een serieuze vraag of een gezellige noot aan het einde van de werkdag. Aurore en Koen, thank you for all the chocolate. Lauren, thank you for the hilarious "TGIF"dances. Mehrdad (Boyke), thank you for the funny remarks you make when we meet in the hallway. Joost, bedankt voor het regelmatig (bijna) slopen van dingen in het lab. Koen, bedankt voor je hulp en adviezen, en succes met het inwijden van je nieuwe drumhok. Theo, bedankt voor het rekken van de labmeetings met je kritische vragen. Jeroen, bedankt voor het in elkaar knutselen van onze AD-converter en het maken van de bijbehorende kabeltjes. Claire, bedankt voor alle dingen die je geregeld hebt en het moedergevoel dat je mij al die jaren hebt gegeven.

Tamara, Sjeng, Maarten, Kujtim en Peter, als oud-studiegenoten, en deels ook ex-collega's vind ik het fijn dat we nog steeds contact hebben. Ik heb genoten van onze tripjes naar elkaars stagebestemming. Hopelijk blijven we contact houden.

Lieve mama, ontzettend bedankt voor alle steun en liefde die jij me mijn hele leven al geeft. Er is niets wat jou tegenhoudt om er voor mij te zijn. Zonder jou en Tom zou ik nooit zo ver gekomen zijn. Tom, bedankt voor alle steun die jij aan mij en aan mam geeft. Ook bedankt voor alle klushulp. Zonder jou hadden Rianne en ik nu niet zo'n mooi huisje.

Allerliefste Rianne, bedankt voor de liefde en het geduld dat je de afgelopen maanden hebt gehad. Zonder blikken of blozen nam jij de regie van alle zaken in en rond ons huis in handen toen ik tot in de late uurtjes aan deze thesis bezig was. Ik hou van jou! 


About the author 


\section{Curriculum vitae}

AOU L Stevens was born on the $5^{\text {th }}$ of November 1990 in Sittard and grew up in Beek. After pre-school he graduated for his Gymnasium degree in 2009 at the Graaf Huyn College in Geleen.

He started to study Biomedical Engineering at the Eindhoven University of Technology where he obtained his Bachelor's degree in 2012.

He continued his study with the Master Medical Engineering also at the university of Eindhoven. During his Master track he performed a four month internship at the Kungliga Tekniska högskolan in Stockholm, where he worked on rupture risk prediction of abdominal aneurysms using computational fluid dynamics models. Following the completion of the foreign internship, he started his master graduation project at the department of Biomedical Engineering at the Maastricht University (UM). Under supervision of Dr. ir. Wouter Huberts he worked on pressure drop estimations of stenoses within the leg arteries using 2D computational models. While working on his graduation project, Raoul received the Dekker student grant from the Dutch Heart association which allowed him to perform an additional internship at the St. Antonius Hospital in Nieuwegein.

After receiving his Master's degree at the Eindhoven University of Technology in 2016, he started his $\mathrm{PhD}$ research on the development of technologies for non-invasive assessment of cerebral pathologies at department of Biomedical Engineering of the UM. Under supervision of Prof. Dr. Tammo Delhaas, Prof. Dr. Werner Mess, and Dr. ir. Wouter Huberts his PhD research lead to the present dissertation. 


\section{Publications}

- Stevens, R.R.F., Donders, W., Quicken, S., van de Vosse, F.N., Mess, W.H., Delhaas, T. \& Huberts, W. The Role of One-Dimensional Model-Generated Inter-Subject Variations in Systemic Properties on Wall Shear Stress Indices of Intracranial Aneurysms. IEEE Trans Biomed Eng. 2020 Apr;67(4):1030-1039.

- van den Boom, T., Stevens, R.R.F., Delhaas, T., van de Vosse, F.N. \& Huberts, W. 0D lumped approach to incorporate the dynamic part of the pressure at vessel junctions in a 1D wave propagation model. International Journal for Numerical Methods in Biomedical Engineering, 34(9).

- Stevens, R.R.F., Gommer, E.D., Ariës, M., Ertl, M., Mess, W.H., Huberts, W. \& Delhaas, T. An Automated Algorithm for the Optic Nerve Sheath Diameter Assessment from B-mode Ultrasound Images (submitted).

- Stevens, R.R.F., Gommer, E.D., Ariës, M., Ertl, M., Mess, W.H., Huberts, W. \& Delhaas, T. The Influence of Discrepancies in Assessment Methodologies on the Optic Nerve Sheath Diameter (submitted).

\section{Not included in this thesis}

- Akyildiz, A.C., Speelman, L., van Velzen, B., Stevens, R.R.F., van der Steen, A.F.W., Huberts, W. \& Gijsen, F.J.H. Intima heterogeneity in stress assessment of atherosclerotic plaques. Interface Focus. 2018;8: 20170008.

doi:10.1098/rsfs.2017.0008

- Stevens, R.R.F., Grytsan, A., Biasetti, J., Roy, J., Liljeqvist, M.L. \& Christian Gasser, T. Biomechanical changes during abdominal aortic aneurysm growth. PLoS One. 2017;12: 1-16. doi:10.1371/journal.pone.0187421 